\title{
General Impulsive Models of Scattering of Mole- cules from a Solid Surface without Tangential Forces
}

\author{
V.M. Azriel', V.M. Akimov, L.I. Kolesnikova, L.Yu. Rusin, M.B. Sevryuk \\ V.L. Talroze Institute for Energy Problems of Chemical Physics at N.N. Semënov Federal Re- \\ search Center for Chemical Physics, Russian Academy of Sciences, Moscow, 119334, Russia \\ azriel_vladimir@mail.ru,vyacheslav-akimov@rambler.ru, ek7787@ rambler.ru, \\ rusin@chph.ras.ru, 2421584@mail.ru
}

\begin{abstract}
We propose a general impulsive model for scattering of a system of atoms or ions from a flat solid surface without tangential forces (that is, forces parallel to the surface). It is assumed within the framework of this model that each individual encounter of an atom with the surface is a series of elastic hits of the atom against surface pseudoparticles, the hits instantly following each other. A distinctive feature of the model is that to each atom, one assigns two infinite sequences of masses of pseudoparticles. Criteria for both finiteness and infinity of series of elastic hits are formulated, based on the masses of the pseudoparticles and the mass of the atom. The model is a far-reaching generalization of the well-known "hard-cube model" for scattering of atoms from a solid surface. We illustrate (by some simplest examples related to scattering of potassium iodide molecules) an application of the correlation analysis to the study of the dependence of the dynamical characteristics of the scattering on the masses of the surface pseudoparticles. The absence of tangential forces seems to be a substantial limitation of the model (for instance, the recent experimental data on scattering of KI from a diamond surface indicate that tangential forces play a significant role in this process).
\end{abstract}

Keywords: scattering of molecules from a surface, impulsive model, surface pseudoparticles, potassium iodide, dissociation, correlation analysis.

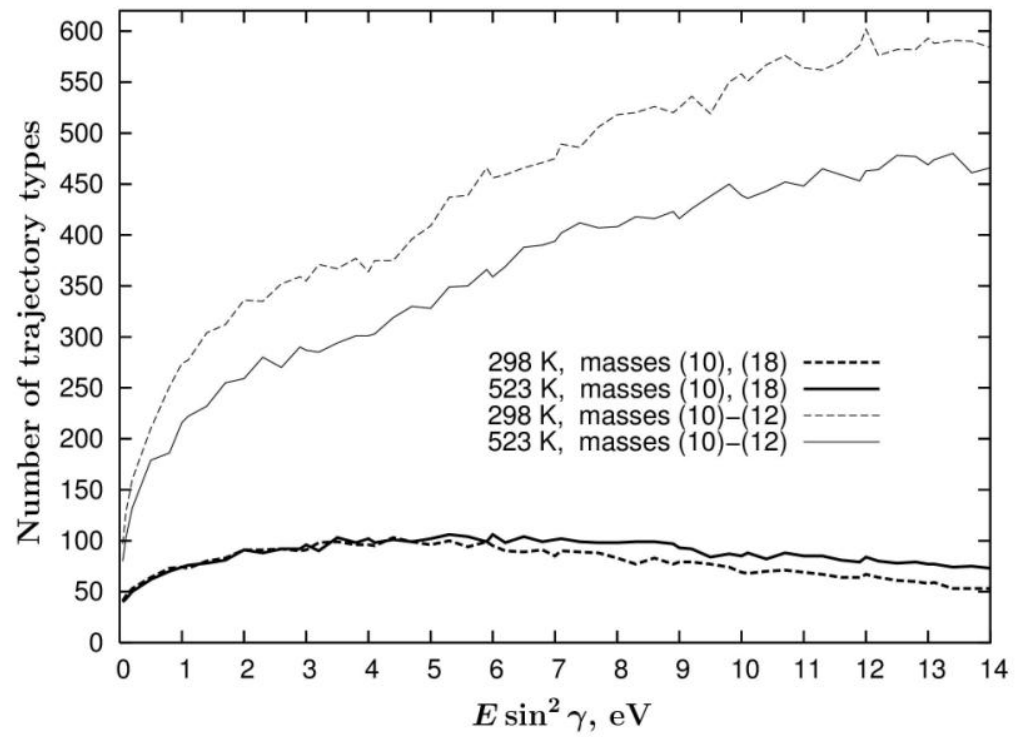

The dependences of the number of trajectory types on the "vertical component" of the energy of molecules in the beam for the two values of the surface temperature

$T_{s}$ and for the two options of the "collisional" and "Maxwellian" masses 


\title{
Общие импульсные модели рассеяния молекул на твердой поверхности без тангенциальных сил
}

\author{
В.М. Азриель, В.М. Акимов, Л.И. Колесникова, Л.Ю. Русин, М.Б. Севрюк \\ Институт энергетических проблем химической физики им. В.Л. Тальрозе Федерального \\ исследовательского иентра химической физики им. Н.Н. Семёнова РАН, \\ Россия, Москва, 119334, Ленинский проспект 38, корп. 2 \\ azriel_vladimir@mail.ru,vyacheslav-akimov@rambler.ru, ek7787@rambler.ru, \\ rusin@chph.ras.ru, 2421584@mail.ru
}

\begin{abstract}
Аннотация
Предложена общая импульсная модель рассеяния системы атомов или ионов на плоской твердой поверхности без тангенциальных сил (т. е. сил, параллельных поверхности). В рамках этой модели предполагается, что каждое отдельное соударение атома с поверхностью представляет собой серию мгновенно следующих друг за другом упругих ударов атома об условные частицы поверхности. Отличительной особенностью модели является то, что каждому атому ставятся в соответствие две бесконечные последовательности масс условных частиц. Сформулированы критерии как конечности, так и бесконечности серий упругих ударов, исходя из масс условных частиц и массы атома. Модель является далеким обобщением известной «модели жестких кубов» рассеяния атомов на твердой поверхности. Проиллюстрировано (на простейших примерах, связанных с рассеянием молекул иодида калия) применение корреляционного анализа к изучению зависимости динамических характеристик рассеяния от масс условных частиц поверхности. Отсутствие тангенциальных сил представляется существенным ограничением модели (например, недавние экспериментальные данные по рассеянию KI на поверхности алмаза показывают, что в этом процессе тангенциальные силы играют значительную роль).
\end{abstract}

Ключевые слова: рассеяние молекул на поверхности, импульсная модель, условные частицы поверхности, иодид калия, диссоциация, корреляционный анализ.

\section{1. Введение}

Импульсное приближение как одно из средств теоретического моделирования различных процессов, происходящих в микромире (в частности, атомно-молекулярных столкновений в газовой фазе или взаимодействия частиц с поверхностью), используется вплоть до настоящего времени. Типичным примером являются импульсные модели трехтельной рекомбинации [1-4]. «Идеология» импульсного приближения в химической физике подробно изложена, например, в работах [3,5-8]. Эти работы посвящены газофазным реакциям, но перечисленные в [3, 6-8] преимущества и недостатки импульсных моделей по сравнению с точными траекторными расчетами в такой же степени характерны и для гетерогенных процессов рассеяния атомов и молекул на поверхности. В основе импульсных моделей лежит идеализация поверхностей потенциальной энергии (ППЭ), управляющих рассматриваемыми процессами, а именно, короткодействующие отталкивающие части потенциалов заменяются бесконечными потенциальными стенками. Во многих случаях ППЭ подвергаются и более радикальным упрощениям, например, используются кусочно-постоянные потенциалы или даже потенциалы жестких сфер. 
Импульсное приближение часто позволяет добиться существенной экономии машинного времени, что было, конечно, чрезвычайно важно в 50-е-70-е годы прошлого века. При нынешнем быстродействии компьютеров этот фактор уже далеко не так значителен, как прежде. Однако такие преимущества классических импульсных моделей перед траекторным счетом на реалистических ППЭ, как физическая наглядность, возможность получить аналитические выражения для некоторых характеристик процесса и отделение эффектов масс взаимодействующих частиц от эффектов рельефа ППЭ («отделение кинематики от динамики»), полностью сохраняют актуальность и сегодня.

Импульсное приближение, как правило, оказывается слишком грубым для того, чтобы на его основе добиться описания экспериментальных данных с хорошей точностью. Однако импульсные аналитические выражения для тех или иных зависимостей часто представляются интересными с методологической точки зрения - подобно тому, как формулы Кардано и Феррари для нахождения (через радикалы) корней уравнений третьей и четвертой степеней соответственно, почти бесполезные в вычислительной практике, имеют колоссальное теоретическое значение. Простейший пример таких аналитических выражений - формула для сечения взаимодействия двух жестких сфер заданного радиуса в пространстве любого числа измерений [1]. В недавней статье [4] мы показали, что даже для такого достаточно сложного процесса, как тримолекулярная реакция рекомбинации $\mathrm{R}+\mathrm{M}+\mathrm{X} \rightarrow \mathrm{R}+\mathrm{MX}$ в трехмерном пространстве, можно предложить содержательную импульсную модель, в рамках которой как сама возможность рекомбинации при данных энергиях столкновения $E_{\mathrm{M}-\mathrm{X}}$ и $E_{\mathrm{R}-\mathrm{MX}}$, так и минимальная энергия молекулы-продукта MX определяются аналитически посредством простых формул. Более того, единственным параметром потенциала взаимодействия рекомбинирующих частиц М и Х, влияющим на функцию эффективности (зависимость минимальной энергии молекулы-продукта от энергий $E_{\mathrm{M}-\mathrm{X}}$ и $\left.E_{\mathrm{R}-\mathrm{MX}}\right)$, оказывается глубина потенциальной ямы $\varepsilon$. «Отделение кинематики от динамики» также представляется полезным приемом при исследовании элементарных процессов. Если данная характеристика динамики процесса сохраняется при переходе от полных квантовомеханических или траекторных расчетов (или же от эксперимента) к импульсной модели, то эта характеристика с высокой степенью достоверности имеет «массовую» природу, т. е. определяется массами взаимодействующих частиц.

В лаборатории Динамики элементарных процессов Института энергетических проблем химической физики имени В.Л. Тальрозе РАН на протяжении нескольких последних лет проводились экспериментальные исследования взаимодействия газодинамического пучка молекул иодида калия с поверхностями графита и алмаза, прежде всего ионной дис-

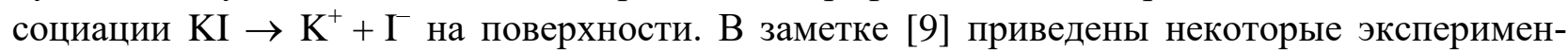
тальные данные по диссоциации молекулы КI на поверхности пиролитического графита; статья, посвященная экспериментам по диссоциации КI на поверхности алмаза (110), принята к публикации в журнале «Химическая физика». Ориентируясь главным образом на эти исследования, мы разработали импульсную модель диссоциации двухатомных молекул с ионной связью (например, молекул галогенидов щелочных металлов) на поверхности графита $[10,11]$. Эта модель во многом основана на широко известной «модели жестких кубов» [12] (см. также [13]) рассеяния атомов на поверхности, но является существенно более сложной: например, в модели $[10,11]$ предусмотрена возможность нескольких упругих ударов иона о т. н. условные частицы поверхности в рамках одного акта соударения иона с поверхностью графита. Отличительной особенностью моделей [10-12] является то, что поверхность считается бесконечной плоскостью, а силы, действующие на атом или ион со стороны поверхности, всегда направлены нормально к последней (так же как и скорость условных частиц). Тангенциальные силы (т. е. силы, параллельные поверхности) отсутствуют, так что компонента полного импульса системы, параллельная поверхности, сохраняется. 
В настоящей работе мы предлагаем новую импульсную модель рассеяния системы атомов или ионов на поверхности без тангенциальных сил. Эта модель является обобщением модели статей $[10,11]$ и содержит (в своем наиболее полном варианте) бесконечно много параметров даже в случае рассеяния одного атома или иона. Подробное описание модели и обсуждение ее некоторых математических аспектов является основной целью работы.

Статья построена следующим образом. В разделе 2 дается краткое изложение «модели жестких кубов» Логана-Стикни [12], обобщением которой являются модели работ $[10,11]$ и модель настоящей статьи. Раздел 3 посвящен т. н. «модели составных соударений» атомов (или ионов) с поверхностью - основному «ингредиенту» того описания взаимодействия молекул с поверхностью, который предлагается в настоящей работе. В «модели составных соударений» предполагается, что каждое отдельное соударение атома с плоской поверхностью представляет собой серию мгновенно следующих друг за другом упругих ударов атома об условные частицы поверхности, причем условная частица, участвующая в $n$-ом ударе, характеризуется парой масс $\left(M_{n}, \mu_{n}\right)$. Такая серия ударов может быть и бесконечной, хотя практическое значение имеют только конечные серии, и в разделе 3 мы доказываем несколько теорем, устанавливающих, в каких случаях (в зависимости от масс условных частиц поверхности и массы самого атома $m$ ) серия ударов заведомо (т. е. с вероятностью 1 ) «обрывается», а в каких она с ненулевой вероятностью является бесконечной. Изложение нашей модели рассеяния молекул на поверхности в рамках метода квазиклассических траекторий заканчивается в разделе 4. В промежутках между соударениями атомов, составляющих данную молекулу, с поверхностью атомы движутся как классические материальные точки, взаимодействие между которыми определяется некоторым потенциалом, а соударения описываются с помощью модели раздела 3. Каждому атому ставится в соответствие своя последовательность масс $M_{1}, \mu_{1}, M_{2}, \mu_{2}, M_{3}, \mu_{3}, \ldots$ Если для некоторой траектории зависимости положений атомов от времени $t$ выражаются функциями $\mathbf{Q}_{j}(t)$ ( $j-$ номер атома), а q и $\mathbf{v}$ - произвольные постоянные векторы, параллельные поверхности, то (благодаря отсутствию тангенциальных сил) функции $\mathbf{Q}_{j}(t)+\mathbf{q}+\mathbf{v} t$ выражают зависимости положений атомов от времени для другой траектории.

В разделе 5 мы напоминаем модель рассеяния молекул КІ на поверхности графита [10, 11], что необходимо для последующего изложения. В разделе 6 обсуждаются другие модели рассеяния молекул иодида калия на твердых поверхностях, отличающиеся от модели статей $[10,11]$ только другим выбором масс условных частиц $\left(M_{n, 1}, \mu_{n, 1}\right)$, отвечающих иону $\mathrm{K}^{+}$, и масс условных частиц $\left(M_{n, 2}, \mu_{n, 2}\right)$, отвечающих иону $\mathrm{I}^{-}$. Полученные нами экспериментальные данные по диссоциативному рассеянию молекул КІ на поверхности алмаза (110) однозначно указывают на значительную роль тангенциальных сил в этом процессе. Поэтому адекватно описать взаимодействие молекул КІ с поверхностью алмаза в рамках модели, предложенной в настоящей работе, невозможно. В то же время остается открытым вопрос, в состоянии ли эта модель удовлетворительно описать (при подходящем выборе масс $\left.M_{n, 1}, M_{n, 2}, \mu_{n, 1}, \mu_{n, 2}\right)$ взаимодействие молекул $\mathrm{KI}$ с поверхностью пиролитического графита. Раздел 7 посвящен т. н. корреляционному анализу зависимости динамических характеристик рассеяния молекул КІ на поверхности от масс условных частиц в предположении, что сами эти массы от $n$ не зависят: $M_{1, j}=M_{2, j}=M_{3, j}=\cdots$ и $\mu_{1, j}=\mu_{2, j}=\mu_{3, j}=\cdots$ $(j=1,2)$. В разделе 8 кратко обсуждается альтернативный подход к описанию рассеяния атомных частиц на поверхности, связанный с концепцией ядра рассеяния. Наконец, основные результаты статьи подытоживаются в разделе 9. 


\section{2. Модель Логана-Стикни}

\section{1. Описание процедуры Логана-Стикни}

В развитии методов теоретического описания рассеяния атомов (или ионов) газа на твердой поверхности важной вехой послужила «модель жестких кубов» (“hard-cube model”) Логана-Стикни [12] (см. также [13]), развивающая более раннюю модель статьи [14]. В рамках «модели жестких кубов» считается, что поверхность представляет собой бесконечную «горизонтальную» плоскость $z=0$ (где $x, y, z$ - декартовы координаты в пространстве) и имеет температуру $T_{s}$, атом массы $m$ движется в полупространстве $z>0$, а его взаимодействие с поверхностью описывается как упругий удар о некоторую условную частицу (“pseudoparticle”) массы $M$ («куб»), скорость которой «вертикальна» (т. е. нормальна к поверхности). Точнее, предположим, что непосредственно перед соударением атома с поверхностью $z$-компонента скорости атома (ортогональная поверхности) равна $u<0$ (отрицательное значение $z$-компоненты скорости атома соответствует приближению к поверхности, а положительное - удалению от нее). В результате соударения компонента скорости атома, параллельная поверхности (т. е. сумма $x$ - и $y$-компонент), остается неизменной, а $z$-компонента мгновенно меняется. Новое значение $u_{\text {new }}$ «вертикальной» компоненты скорости атома определяется с помощью алгоритма, который мы будем называть процедурой $L S\left(m, M, T_{s}, u\right)$ (от Logan-Stickney). Эта процедура состоит в следующем.

1) Разыгрывается абсолютная величина $U_{*}>0$ скорости условной частицы поверхности непосредственно перед соударением, исходя из одномерного максвелловского распределения, отвечающего температуре $T_{s}$ (измеряемой в градусах Кельвина). Иначе говоря, плотность вероятности случайной величины $U_{*}$ равна

$$
\left(\frac{2 M}{\pi k_{\mathrm{B}} T_{s}}\right)^{1 / 2} \exp \left(-\frac{M U_{*}^{2}}{2 k_{\mathrm{B}} T_{s}}\right),
$$

где $k_{\mathrm{B}}$ - постоянная Больцмана. Сама скорость частицы при этом есть $U= \pm U_{*}$.

2) Знак скорости $U$ выбирается по следующему правилу. Обозначим через $u_{*}=|u|=-u$ абсолютную величину $z$-компоненты скорости атома непосредственно перед соударением. Если $u_{*} \leq U_{*}$, то частица движется навстречу атому: $U=U_{*}>0$. Если же $u_{*}>U_{*}$, то с вероятностью $\left(u_{*}+U_{*}\right) /\left(2 u_{*}\right)>1 / 2$ частица движется навстречу атому: $U=U_{*}>0$, а с вероятностью $\left(u_{*}-U_{*}\right) /\left(2 u_{*}\right)<1 / 2$ частица «убегает» от атома: $U=-U_{*}<0$. При этом во всех случаях $U>u$ (условие возможности удара), так как $u<0$, а скорость $U$ может быть отрицательной только в случае, когда $|U|=U_{*}<u_{*}=|u|$ (это рассуждение «проходит» и при $u=0)$.

Другими словами, плотность вероятности случайной величины $U>-u_{*}=u$ равна

$$
\left(\frac{2 M}{\pi k_{\mathrm{B}} T_{s}}\right)^{1 / 2} \min \left(\frac{u_{*}+U}{2 u_{*}}, 1\right) \exp \left(-\frac{M U^{2}}{2 k_{\mathrm{B}} T_{s}}\right)
$$

(с той оговоркой, что $U$ не может принимать нулевое значение).

3) Происходит мгновенный упругий удар атома об условную частицу поверхности, в результате которого $z$-компонента скорости атома становится равной

$$
u_{\text {new }}=\frac{(m-M) u+2 M U}{m+M}>u .
$$


Новое значение скорости частицы поверхности

$$
U_{\text {new }}=\frac{(M-m) U+2 m u}{m+M}<U
$$

для этой модели несущественно. Отметим неравенство $u_{\text {new }}>U_{\text {new }}$ (эквивалентное неравенству $U>u)$ и соотношения $m u+M U=m u_{\text {new }}+M U_{\text {new }}$ (сохранение импульса) и $m u^{2}+M U^{2}=m u_{\text {new }}^{2}+M U_{\text {new }}^{2}$ (сохранение энергии). Формулы (1) и (2) можно переписать в виде

$$
u_{\text {new }}-u=\frac{2 M}{m+M}(U-u)>0, \quad U_{\text {new }}-U=\frac{2 m}{m+M}(u-U)<0 .
$$

Количество обращений к датчику случайных чисел в рамках процедуры $L S\left(m, M, T_{s}, u\right)$ равно (при наиболее «естественной» программной реализации этой процедуры) 1 или 2. Сначала датчик случайных чисел используется для розыгрыша величины $U_{*}$. Если $u_{*}>U_{*}$, то для определения знака скорости $U$ осуществляется повторное обращение к датчику случайных чисел.

Так как скорость $U$ может принимать любое значение из бесконечного интервала $u<U<\infty$ (кроме значения $U=0$ ), $z$-компонента $u_{\text {new }}$ скорости атома после соударения, вычисляемая по формуле (1), может принимать любое значение из бесконечного интервала $u<u_{\text {new }}<\infty$, кроме значения $(m-M) u /(m+M)$.

Чтобы атом «отлетел» от поверхности, значение $u_{\text {new }}$ должно быть положительным. Авторы статьи [12] предполагают, что $M>3 m$ (в ключевом пункте IV-A работы) или что $M>m$ (в пункте IV-B), рассматривают в основном усредненные величины

$$
\left\langle u_{\text {new }}\right\rangle=\frac{(m-M) u+2 M\langle U\rangle}{m+M}
$$

(фактически в [12] проводится еще усреднение по $u$, исходя из температуры $T_{g}$ пучка газа над поверхностью) и тем самым «обходят» анализ ситуации, когда $u_{\text {new }} \leq 0,-$ несмотря на то, что вероятность этой ситуации, т. е. вероятность того, что скорость $U$ лежит в интервале $u<U \leq(M-m) u /(2 M)$, положительна при любых значениях $m, M, T_{s}$ и $u$. Ситуацию, когда $u_{\text {new }} \leq 0$, можно (несколько условно) рассматривать как адсорбцию атома поверхностью.

\section{2. Дальнейшее развитие модели}

«Модель жестких кубов» очень проста, ее даже иногда называют «рудиментарной» (“rudimentary") [15]. Однако многие последующие модели рассеяния атомов или молекул на поверхности твердого тела развивают «модель жестких кубов». Например, в работах [16, 17] эта модель дополнена притягивающим потенциалом, равным нулю при $z<z_{0}$ и равным $W$ при $z>z_{0}$, где $z_{0}$ и $W$ - положительные константы (впрочем, значение $z_{0}$ несущественно). В «модели мягких кубов» (“soft-cube model”) [18] атомы поверхности представляются одномерными гармоническими осцилляторами, которые колеблются перпендикулярно поверхности, а взаимодействие атома газа с осциллятором описывается потенциалом, состоящим из экспоненциальной отталкивающей части и последующей конечной ступеньки. Иначе говоря, если $z$ - расстояние до осциллятора, а $z_{\bullet}$ - отклонение осциллятора от равновесного положения, то рассматриваемый потенциал равен $A e^{-z / \rho}$ при $z+z_{\bullet}<z_{0}$ и ра- 
вен $W$ при $z+z_{\bullet}>z_{0}$, где $z_{0}, A, \rho$ и $W$ - положительные константы. В «модели жестких сфероидов» (“hard-spheroid model”) $[19,20]$, в отличие от «модели жестких кубов», условные частицы поверхности снабжены сферическими «шапочками» (“caps”) с заданным углом раствора $2 \alpha$, и атомы газа взаимодействуют импульсным образом с этими «шапочками».

В очень популярной «модели стиральной доски» (“washboard model”) [21] (см. также [22]), близкой к «модели жестких сфероидов», также предполагается импульсное взаимодействие атомов газа с условными частицами поверхности, как и в модели Логана-Стикни, но сама поверхность является «гофрированной» (“corrugated”), т. е. складчатой, и описывается не уравнением $z=0$, а уравнением $z=\zeta(x)=A \cos (2 \pi x / a)$, где $A$ и $a$ - положительные параметры модели. Если соударение атома с поверхностью происходит в точке $(x, y, \zeta(x))$, то компонента скорости атома, нормальная к поверхности в этой точке, т. е. коллинеарная вектору $\left(-\zeta^{\prime}(x), 0,1\right)$, претерпевает мгновенное изменение, а компонента скорости, параллельная касательной к поверхности в этой точке, сохраняется. Модель включает также притягивающий потенциал, равный нулю при $z<z_{0}$ и равный $W$ при $z>z_{0}$, где $z_{0}>A$ и $W \geq 0$ - константы. «Гофры» другой формы, т. е. случай других периодических функций $\zeta$, рассмотрен в статье [23]. В работе [24] было предложено обобщение «модели стиральной доски», в котором условные частицы поверхности характеризуются не только массой $M$, но и моментом инерции $I$, и в рассмотрение вводится прицельный параметр $b$ атома газа относительно центра масс частицы поверхности (но компонента скорости атома, параллельная касательной к поверхности в точке соударения, по-прежнему сохраняется). Статья [15] посвящена другому обобщению «модели стиральной доски». В этой работе поверхность описывается набором круговых гауссовых «холмов» (“hills") и «долин» (“valleys”) со случайным расположением и случайной высотой/глубиной, т. е. задается уравнением

$$
z=S_{N}(x, y)=\frac{1}{(2 \pi)^{1 / 2} \sigma} \sum_{i=1}^{N} C_{i} \exp \left(-\frac{\left(x-x_{i}\right)^{2}+\left(y-y_{i}\right)^{2}}{2 \sigma^{2}}\right),
$$

где $\sigma>0$, а случайно выбираемые числа $x_{i}, y_{i}$ и $C_{i} \neq 0$ могут быть любого знака. Множитель $(2 \pi)^{-1 / 2} \sigma^{-1}$ выбран из соображений удобства вычисления среднего $\left\langle S_{N}^{2}\right\rangle$.

Модель статьи [25] отличается от «модели жестких кубов» тем, что в [25] газ над поверхностью состоит не из материальных точек, а из жестких ротаторов, вращающихся в «вертикальных» плоскостях и представляющих двухатомные гомоядерные молекулы. В похожей «модели жестких кубов и жестких эллипсоидов с трением» (“frictional hard-cube hardellipsoid model”) [26] гомоядерные молекулы газа представляются жесткими эллипсоидами, и при этом присутствуют тангенциальные силы (силы трения). В статье [22] рассматриваются столкновения жестких эллипсоидов с «гофрированной» поверхностью. В литературе известны и другие обобщения модели Логана-Стикни, предназначенные для описания рассеяния на поверхности двухатомных гомоядерных $[27,28]$ и гетероядерных [29] молекул (а также, например, многоатомных сферических молекул [30] при наличии тангенциальных сил).

Подчеркнем, что мы перечислили в этом подразделе лишь некоторые ключевые (или наиболее характерные) обобщения «модели жестких кубов». Из обзоров по рассеянию атомов, ионов и молекул на твердой поверхности, в которых рассматриваются «модель жестких кубов» и ее обобщения, отметим, например, [13, 31-38] (все эти обзоры легко доступны в Интернете). Автор заметки [39] выдвинул предположение, что у этих моделей есть квантовомеханический базис. 


\section{3. «Модель составных соударений»}

\section{1. Процедура Логана-Стикни с двумя массами условной частицы поверхности}

Центральной составной частью нашей модели взаимодействия частицы с твердой поверхностью («модели составных соударений», в качестве соответствующего английского термина можно было бы взять “composite encounter model”) является обобщение процедуры Логана-Стикни $L S\left(m, M, T_{s}, u\right)$, которое мы будем обозначать через $L S_{2}\left(m, M, \mu, T_{s}, u\right)$ и в котором условная частица поверхности характеризуется двумя массами $M$ и $\mu$ (о чем напоминает нижний индекс «2»), а $u \leq 0$. В рамках процедуры $L S_{2}\left(m, M, \mu, T_{s}, u\right)$ значение $z$-компоненты скорости атома непосредственно после соударения с поверхностью попрежнему определяется формулой (1), но абсолютная величина $U_{*}$ скорости $U$ условной частицы поверхности непосредственно перед соударением разыгрывается, исходя из одномерного максвелловского распределения, отвечающего температуре $T_{s}$ и массе $\mu:$ плотность вероятности случайной величины $U_{*}$ равна

$$
\left(\frac{2 \mu}{\pi k_{\mathrm{B}} T_{s}}\right)^{1 / 2} \exp \left(-\frac{\mu U_{*}^{2}}{2 k_{\mathrm{B}} T_{s}}\right) \text {. }
$$

Иначе говоря, масса $M$ служит для описания упругого удара атома и условной частицы поверхности, а масса $\mu$ - для описания распределения скорости условной частицы поверхности до удара. Знак скорости $U$ выбирается так же, как в процедуре Логана-Стикни. Исходная процедура $L S\left(m, M, T_{s}, u\right)$ есть $L S_{2}\left(m, M, M, T_{s}, u\right)$.

Вероятность того, что $U_{*} \leq \Gamma$ для какого-либо положительного числа $\Gamma$, равна

$$
\left(\frac{2 \mu}{\pi k_{\mathrm{B}} T_{s}}\right)^{1 / 2} \int_{0}^{\Gamma} \exp \left(-\frac{\mu U_{*}^{2}}{2 k_{\mathrm{B}} T_{s}}\right) \mathrm{d} U_{*}=\operatorname{erf}\left(\left[\frac{\mu}{2 k_{\mathrm{B}} T_{s}}\right]^{1 / 2} \Gamma\right),
$$

где

$$
\operatorname{erf}(t)=\frac{2}{\pi^{1 / 2}} \int_{0}^{t} e^{-\tau^{2}} \mathrm{~d} \tau
$$

- функция ошибок (когда $t$ изменяется от нуля до бесконечности, $\operatorname{erf}(t)$ монотонно возрастает от 0 до 1). Вероятность того, что $U_{*} \geq \Gamma$, равна

$$
\left(\frac{2 \mu}{\pi k_{\mathrm{B}} T_{s}}\right)^{1 / 2} \int_{\Gamma}^{\infty} \exp \left(-\frac{\mu U_{*}^{2}}{2 k_{\mathrm{B}} T_{s}}\right) \mathrm{d} U_{*}=1-\operatorname{erf}\left(\left[\frac{\mu}{2 k_{\mathrm{B}} T_{s}}\right]^{1 / 2} \Gamma\right) .
$$

В общем случае масса атома $m$ положительна и конечна, «столкновительная» масса $M$ условной частицы поверхности неотрицательна и может быть бесконечной, а «максвелловская» масса $\mu$ условной частицы поверхности положительна и может быть бесконечной.

Строгие неравенства в формулах (1) и (2) отвечают ситуации, когда $0<M<\infty$. Если $M=0$, то скорость атома при ударе о частицу поверхности не меняется:

$$
u_{\text {new }}=u, \quad U_{\text {new }}=2 u-U<U .
$$

Если же $M=\infty$, то при ударе не меняется скорость частицы поверхности: 


$$
u_{\text {new }}=2 U-u>u, \quad U_{\text {new }}=U .
$$

В обоих случаях по-прежнему $u_{\text {new }}>U_{\text {new }}$ (так как $U>u$ ).

Бесконечность массы $\mu$ означает, что $U=U_{*}=0$ (независимо от $T_{s}$ ), так что датчик случайных чисел для определения $U$ не требуется и

$$
u_{\text {new }}=\frac{(m-M) u}{m+M}, \quad U_{\text {new }}=\frac{2 m u}{m+M} .
$$

Если $\mu=\infty$ и $u=0$, то $u=U=u_{\text {new }}=U_{\text {new }}=0$. Это единственная ситуация, когда $U=u$ и $u_{\text {new }}=U_{\text {new }}$; во всех остальных случаях $U>u$ и $u_{\text {new }}>U_{\text {new }}$.

Отметим, что увеличение массы $M$ в $d$ раз $(0<d<\infty)$ эквивалентно уменьшению массы $m$ в $d$ раз, потому что в формулы (1) и (2) входит только отношение $M / m$. Аналогичным образом, увеличение массы $\mu$ в $d$ раз эквивалентно уменьшению температуры $T_{s}$ в $d$ раз, потому что в выражение (3) входит только отношение $\mu / T_{s}$.

\section{2. Описание процедуры составных соударений}

Главной идеей «модели составных соударений» является то, что ситуацию, когда $u_{\text {new }} \leq 0$, надо не игнорировать, а рассматривать как основание для усложнения процедуры. Модель базируется на следующих принципах.

a) Соударение атома (или иона) с поверхностью представляет собой серию мгновенно следующих друг за другом упругих ударов атома об условные частицы поверхности. Каждый удар описывается обобщенной процедурой Логана-Стикни (с двумя массами). Серия прекращается, как только $z$-компонента скорости атома становится положительной (т. е. атом «отлетает» от поверхности).

б) Условная частица поверхности, участвующая в $n$-ом упругом ударе, характеризуется своей «столкновительной» массой $M_{n}$ и своей «максвелловской» массой $\mu_{n}$. Массы $M_{n}$ для разных $n$ не обязаны совпадать, равно как и массы $\mu_{n}$ для разных $n$. Скорости частиц поверхности, участвующих в разных упругих ударах (скорости $U_{n}$ для разных $n$ ), разыгрываются независимо.

Дадим теперь формальное описание «модели составных соударений». Твердая поверхность в рамках этой модели снова представляет собой бесконечную плоскость $z=0$ и имеет некоторую температуру $T_{s}$, а атом (или ион) массы $m$ движется в полупространстве $z>0$ (оба значения $m$ и $T_{s}$ положительны и конечны). Взаимодействие атома с поверхностью характеризуется двумя последовательностями масс:

$$
\left\{M_{n}\right\}_{n \geq 1}=\left(M_{1}, M_{2}, M_{3}, \ldots\right), \quad 0 \leq M_{n} \leq \infty
$$

И

$$
\left\{\mu_{n}\right\}_{n \geq 1}=\left(\mu_{1}, \mu_{2}, \mu_{3}, \ldots\right), \quad 0<\mu_{n} \leq \infty .
$$

Непосредственно перед соударением атома с поверхностью $z$-компонента скорости атома («вертикальная» компонента) равна $u_{0}<0$. В результате соударения компонента скорости атома, параллельная поверхности («горизонтальная» компонента), остается неизменной, а $z$-компонента мгновенно меняется. Новое значение $u_{\perp}$ «вертикальной» компоненты скорости атома определяется с помощью алгоритма, который мы будем называть процедурой $C E\left(m,\left\{M_{n}\right\},\left\{\mu_{n}\right\}, T_{s}, u_{0}\right)$ (от “composite encounter”). Эта процедура состоит в следующем. 
В рамках процедуры $L S_{2}\left(m, M_{1}, \mu_{1}, T_{s}, u_{0}\right)$ определяется $z$-компонента $u_{1}$ скорости атома после первого упругого удара об условную частицу поверхности. Если $u_{1}>0$, то $u_{\perp}=u_{1}$ (после первого же удара атом «отлетает» от поверхности, и всё соударение атома с поверхностью сводится к этому удару). Если же $u_{1} \leq 0$ (после первого удара атом продолжает «вдавливаться» в твердое тело, ограниченное поверхностью), то за первым ударом сразу же следует второй, причем $z$-компонента $u_{2}$ скорости атома после второго упругого удара об условную частицу поверхности определяется в рамках процедуры $L S_{2}\left(m, M_{2}, \mu_{2}, T_{s}, u_{1}\right)$. Если $u_{2}>0$, то $u_{\perp}=u_{2}$ (соударение атома с поверхностью сводится к первым двум ударам). Если же $u_{2} \leq 0$, то за вторым ударом сразу же следует третий, а $z$-компонента $u_{3}$ скорости атома после третьего упругого удара об условную частицу поверхности определяется в рамках процедуры $L S_{2}\left(m, M_{3}, \mu_{3}, T_{s}, u_{2}\right)$, и т. д.

Более формально, перед $n$-ым ударом $(n \geq 1)$ атома об условную частицу поверхности $z$-компонента скорости атома равна $u_{n-1} \leq 0$. В результате удара $z$-компонента скорости атома приобретает значение $u_{n}$, определяемое в рамках процедуры $L S_{2}\left(m, M_{n}, \mu_{n}, T_{s}, u_{n-1}\right)$ (скорость условной частицы поверхности, которая разыгрывается при этой процедуре, мы будем обозначать через $U_{n}$ ). Если $u_{n}>0$, то серия ударов прекращается, и окончательное значение $z$-компоненты скорости атома после соударения с поверхностью равно $u_{\perp}=u_{n}$. Если же $u_{n} \leq 0$, то сразу же после $n$-ого удара происходит $(n+1)$-ый удар.

Отметим, что одновременное увеличение всех масс $M_{1}, M_{2}, M_{3}, \ldots$ в $d$ раз $(0<d<\infty)$ эквивалентно уменьшению массы $m$ в $d$ раз, а одновременное увеличение всех масс $\mu_{1}, \mu_{2}, \mu_{3}, \ldots$ в $d$ раз эквивалентно уменьшению температуры $T_{s}$ в $d$ раз.

Естественный и очень важный вопрос, который встает при определении процедуры $C E\left(m,\left\{M_{n}\right\},\left\{\mu_{n}\right\}, T_{s}, u_{0}\right)$, состоит в том, в каких случаях эта процедура действительно «обрывается» после конечного числа ударов атома об условные частицы поверхности, т. е. $z$-компонента скорости атома становится положительной и он «отлетает» от поверхности.

\section{3. Бесконечные серии ударов}

Легко указать ситуации, когда серия ударов атома заведомо бесконечна. Например, это так, если $M_{n}=0$ при всех $n \geq 1$, потому что в этом случае $u_{n}=u_{0}<0$ при всех $n$, или если $\mu_{n}=\infty$ и $M_{n} \leq m$ при всех $n \geq 1$, потому что в этом случае

$$
u_{n}=\frac{\left(m-M_{n}\right) u_{n-1}}{m+M_{n}} \leq 0
$$

при всех $n \geq 1$. Если все «максвелловские» массы $\mu_{n}$ конечны, то для любой последовательности «столкновительных» масс $M_{n}$ и любой скорости $u_{0}<0$ можно указать последовательность ненулевых скоростей $U_{n}>u_{n-1}$, для которой скорость $u_{n}$ будет отрицательной при всех $n$ и, таким образом, соответствующая серия ударов окажется бесконечной. Действительно, пусть $u_{n-1}<0$ для данного $n \geq 1$. Если $M_{n}=0$, то $u_{n}=u_{n-1}<0$ при любой скорости $U_{n}$. Если $M_{n}=\infty$, то, положив $U_{n}=\frac{3}{4} u_{n-1}>u_{n-1}$, получим $u_{n}=2 U_{n}-u_{n-1}=\frac{1}{2} u_{n-1}<0$. Если же $0<M_{n}<\infty$, но $3 M_{n} \neq m$, то, положив

$$
U_{n}=\frac{3 M_{n}-m}{4 M_{n}} u_{n-1}>u_{n-1},
$$


получим

$$
u_{n}=\frac{\left(m-M_{n}\right) u_{n-1}+2 M_{n} U_{n}}{m+M_{n}}=\frac{u_{n-1}}{2}<0 .
$$

Наконец, если $M_{n}=m / 3$, то можно взять любое ненулевое значение $U_{n}$ из интервала $u_{n-1}<U_{n}<-u_{n-1}$; тогда $u_{n}=\left(u_{n-1}+U_{n}\right) / 2<0$.

Более того, справедлива следующая теорема. Для любой последовательности «столкновительных» масс $\left\{M_{n}\right\}_{n \geq 1}$, все члены которой меньше $m$, любой константы $\eta<0$, любого числа $p$ из интервала $0<p<1$ и любой температуры $T_{s}$ найдется последовательность конечных «максвелловских» масс $\left\{\mu_{n}\right\}_{n \geq 1}$, для которой серия ударов в рамках процедуры $C E\left(m,\left\{M_{n}\right\},\left\{\mu_{n}\right\}, T_{s}, u_{0}\right)$ будет бесконечной с вероятностью не менее $p$ для любой начальной скорости $u_{0} \leq \eta$.

Действительно, предположим сначала, что все массы $M_{n}$ положительны. Определим последовательности отрицательных чисел $\left\{\kappa_{n}\right\}_{n \geq 0}$ и положительных чисел $\left\{\Gamma_{n}\right\}_{n \geq 1}$ следующими соотношениями:

$$
\begin{gathered}
\kappa_{0}=\eta, \quad \kappa_{n}=\frac{m-M_{n}}{2\left(m+M_{n}\right)} \kappa_{n-1} \text { при } n \geq 1, \\
\Gamma_{n}=-\frac{m+M_{n}}{2 M_{n}} \kappa_{n} \text { при } n \geq 1 .
\end{gathered}
$$

Кроме того, пусть $\left\{\delta_{n}\right\}_{n \geq 1}$ - произвольная монотонно строго убывающая последовательность чисел в интервале $p<\delta_{n}<1$, например,

$$
\delta_{n}=p+\frac{1-p}{n+1}=\frac{n p+1}{n+1} .
$$

Положим

$$
\chi_{1}=\delta_{1}<1, \quad \chi_{n+1}=\frac{\delta_{n+1}}{\delta_{n}}<1 \text { при } n \geq 1
$$

и определим последовательность положительных чисел $\left\{\mu_{n}\right\}_{n \geq 1}$ соотношениями

$$
\operatorname{erf}\left(\left[\frac{\mu_{n}}{2 k_{\mathrm{B}} T_{s}}\right]^{1 / 2} \Gamma_{n}\right)=\chi_{n}
$$

(см. (4)). Числа $\mu_{n}$ определены корректно, так как $\Gamma_{n}>0$ и $0<\chi_{n}<1$. Если $u_{n-1} \leq \kappa_{n-1}$ и $U_{n} \leq \Gamma_{n}$ при некотором $n \geq 1$, то $u_{n} \leq \kappa_{n}<0$. В самом деле,

$$
u_{n}=\frac{\left(m-M_{n}\right) u_{n-1}+2 M_{n} U_{n}}{m+M_{n}} \leq \frac{\left(m-M_{n}\right) \kappa_{n-1}+2 M_{n} \Gamma_{n}}{m+M_{n}}=\frac{2\left(m+M_{n}\right) \kappa_{n}-\left(m+M_{n}\right) \kappa_{n}}{m+M_{n}}=\kappa_{n} .
$$

Таким образом, если $u_{0} \leq \kappa_{0}=\eta$ и $U_{n} \leq \Gamma_{n}$ при всех $n \geq 1$, то $u_{n} \leq \kappa_{n}<0$ при всех $n \geq 0$, и серия ударов никогда не «оборвется». Но вероятность того, что $U_{n} \leq \Gamma_{n}$ для данного $n$, не меньше вероятности того, что $\left|U_{n}\right| \leq \Gamma_{n}$, а последняя вероятность согласно формулам (4) и (6) равна $\chi_{n}$. Значит, вероятность того, что $U_{n} \leq \Gamma_{n}$ при всех $n \geq 1$, не меньше 


$$
\prod_{n=1}^{\infty} \chi_{n}=\delta_{1} \prod_{n=2}^{\infty} \frac{\delta_{n}}{\delta_{n-1}}=\lim _{n \rightarrow \infty} \delta_{n} \geq p
$$

Если же $M_{n}=0$ для некоторого $n \geq 1$, то $\kappa_{n}=\kappa_{n-1} / 2$, а в качестве $\Gamma_{n}$ можно взять любое положительное число. По-прежнему, если $u_{n-1} \leq \kappa_{n-1}$, то

$$
u_{n}=u_{n-1} \leq \kappa_{n-1}=2 \kappa_{n}<\kappa_{n} \text {. }
$$

\section{4. Конечные серии ударов}

«Игра» в теореме в конце предыдущего подраздела идет на том, что все «столкновительные» массы $M_{n}$ меньше массы атома $m$, а последовательность «максвелловских» масс $\mu_{n}$ может неограниченно возрастать. Укажем две ситуации, когда заведомо (т. е. с вероятностью 1$)$ серия ударов в рамках процедуры $C E\left(m,\left\{M_{n}\right\},\left\{\mu_{n}\right\}, T_{s}, u_{0}\right)$ «оборвется» при любой начальной скорости $u_{0} \leq 0$.

1) $M_{n} \geq m$ и $\mu_{n}<\infty$ при всех достаточно больших $n$ (скажем, при $n \geq n_{o}$ ). Действительно, предположим, что все скорости $u_{n}(n \geq 0)$ при этом неположительны. Так как $U_{n}>0$ с вероятностью, превышающей $1 / 2$ (если только $\mu_{n}<\infty$ ), заведомо найдется такое $n \geq n_{o}$, для которого $U_{n}>0$. Тогда из $u_{n-1} \leq 0$ и $M_{n} \geq m$ вытекает, что

$$
u_{n}=\frac{\left(m-M_{n}\right) u_{n-1}+2 M_{n} U_{n}}{m+M_{n}}>0 .
$$

В этой формуле мы предполагаем, что масса $M_{n}$ конечна. Если же $M_{n}=\infty$, то $u_{n}=2 U_{n}-u_{n-1}>0$.

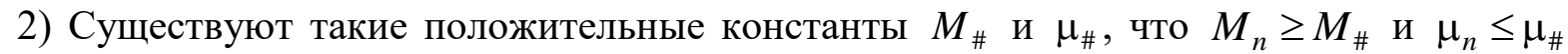
при всех $n \geq 1$. Действительно, предположим, что все скорости $u_{n}(n \geq 0)$ при этом неположительны. Зафиксируем произвольное число $\Gamma>0$. Вероятность того, что $\mid U_{n} \geq \Gamma$ для данного $n$, согласно (5) равна

$$
1-\operatorname{erf}\left(\left[\frac{\mu_{n}}{2 k_{\mathrm{B}} T_{s}}\right]^{1 / 2} \Gamma\right) \geq 1-\operatorname{erf}\left(\left[\frac{\mu_{\#}}{2 k_{\mathrm{B}} T_{s}}\right]^{1 / 2} \Gamma\right)>0
$$

Вероятность же того, что $U_{n} \geq \Gamma$, больше половины этой величины. Следовательно, последовательность $\left\{U_{n}\right\}_{n \geq 1}$ заведомо содержит бесконечно много членов, не меньших $Г$, а потому

$$
\sum_{n=1}^{\infty} \max \left(U_{n}, 0\right)=\infty
$$

Так как $M_{n} \geq M_{\#}, u_{n-1} \leq 0$ и $U_{n}>u_{n-1}$ при всех $n \geq 1$, то

$$
u_{n}-u_{0}=\sum_{\mathrm{\imath}=1}^{n}\left(u_{\mathrm{\imath}}-u_{\mathrm{\imath}-1}\right)=\sum_{\mathrm{\imath}=1}^{n} \frac{2 M_{\mathrm{\imath}}}{m+M_{\mathrm{\imath}}}\left(U_{\mathrm{\imath}}-u_{\mathrm{\imath}-1}\right) \geq \frac{2 M_{\text {\# }}}{m+M_{\#}} \sum_{\mathrm{\imath}=1}^{n}\left(U_{\mathrm{\imath}}-u_{\mathrm{\imath}-1}\right) \geq \frac{2 M_{\#}}{m+M_{\#}} \sum_{\mathrm{i}=1}^{n} \max \left(U_{\mathrm{\imath}}, 0\right),
$$


а последнее выражение стремится к бесконечности при $n \rightarrow \infty$. Значит, $u_{n}>0$ при достаточно больших $n$. Если $M_{\imath}=\infty$ для некоторого 1 , то вместо $2 M_{1} /\left(m+M_{\imath}\right)$ в этой выкладке следует брать коэффициент 2.

Эти две ситуации охватывают, по-видимому, все практически значимые варианты выбора «столкновительных» и «максвелловских» масс.

Интересно, что в ранней статье [14] соударение атома с поверхностью также описывалось как серия следующих друг за другом упругих ударов атома газа (массы $m$ ) об условные частицы (атомы) поверхности (массы $M$ ). В работе [14] предполагается, что атомы как газа, так и поверхности движутся нормально к поверхности, причем атом поверхности заключен в одномерный «ящик», непроницаемый для него, но абсолютно проницаемый для атома газа, и упруго отражается от концов «ящика». Удары атома газа по атому поверхности также упругие. В отличие от нашей модели, скорость $U_{n}$ атома поверхности перед $n$-ым ударом $(n \geq 2)$ двух атомов однозначно определяется скоростью атома поверхности $U_{\text {new }, n-1}$ после $(n-1)$-ого удара: $U_{n}=-U_{\text {new }, n-1}>0$ (атом поверхности отразился от «нижнего» конца «ящика»). Серия ударов заканчивается $n$-ым ударом $(n \geq 1)$, если скорость атома газа $u_{n}$ после этого удара удовлетворяет неравенству $u_{n} \geq\left|U_{\text {new }, n}\right|$ (ось координат направлена от поверхности). Так как всегда $u_{n}>U_{\text {new }, n}$, то неравенство $u_{n} \geq\left|U_{\text {new }, n}\right|$ эквивалентно неравенству $u_{n} \geq-U_{\text {new, } n}$. В статье [14] проанализирован только случай $M>m$, когда соударение может включать максимум два удара атомов.

Доказательство того факта, что при $M>m$ (и даже при $M \geq m$ ) соударение не может включать три удара атомов, очень простое. Предположим, что второй удар имеет место. Согласно формулам (1) и (2) неравенство $u_{2} \geq-U_{\text {new,2 }}$ имеет вид

$$
\frac{(m-M) u_{1}+2 M U_{2}}{m+M} \geq-\frac{(M-m) U_{2}+2 m u_{1}}{m+M},
$$

T. e.

$$
(3 m-M) u_{1}+(3 M-m) U_{2} \geq 0 .
$$

Однако $U_{2}=-U_{\text {new }, 1}>0, u_{1}>U_{\text {new }, 1}=-U_{2}$ и $u_{1}<-U_{\text {new }, 1}=U_{2}$ (иначе второго удара не было бы). Левая часть неравенства (7) является многочленом первой степени от $u_{1}$, и для проверки этого неравенства на интервале $-U_{2}<u_{1}<U_{2}$ достаточно проверить неравенство (7) на концах этого интервала. При $u_{1}=-U_{2}$ получаем $4(M-m) U_{2} \geq 0$, так как $M \geq m$ и $U_{2}>0$. При $u_{1}=U_{2}$ получаем $2(m+M) U_{2}>0$.

В работе [24] подробно рассмотрены множественные соударения атома с поверхностью, но совершенно другой природы: каждое соударение представляет собой единственный удар атома по условной частице поверхности (с ненулевым моментом инерции), но в силу выраженной «гофрированности» поверхности атом, «отлетев» от поверхности, может столкнуться с ней вновь в другой точке.

\section{4. Рассеяние молекул на поверхности}

\section{1. Общее описание подхода}

Рассмотрим теперь молекулу, состоящую из атомов (или ионов в случае ионных связей) $\mathrm{A}_{1}, \mathrm{~A}_{2}, \ldots$ Описание рассеяния такой молекулы на твердой поверхности с помощью «модели составных соударений» в рамках метода квазиклассических траекторий осуществ- 
ляется следующим образом. Каждому атому $\mathrm{A}_{j}$ ставятся в соответствие его масса $m_{j}>0$, радиус $R_{j} \geq 0$ и две последовательности масс

$$
\left\{M_{n, j}\right\}_{n \geq 1}=\left(M_{1, j}, M_{2, j}, M_{3, j}, \ldots\right), \quad 0 \leq M_{n, j} \leq \infty
$$

и

$$
\left\{\mu_{n, j}\right\}_{n \geq 1}=\left(\mu_{1, j}, \mu_{2, j}, \mu_{3, j}, \ldots\right), \quad 0<\mu_{n, j} \leq \infty,
$$

характеризующих взаимодействие этого атома с поверхностью (конечно, предполагается, что выбор этих масс физически мотивирован). Сама поверхность представляет собой бесконечную плоскость $z=0$ и имеет некоторую температуру $T_{s}$, а атомы движутся в полупространстве $z>0$. Атом $\mathrm{A}_{j}$ сталкивается с поверхностью как твердый шарик радиуса $R_{j}$. Пока расстояние от ядра каждого атома до поверхности (т. е. $z$-координата ядра атома) больше радиуса этого атома, движение системы атомов моделируется как движение классических материальных точек (с массами $m_{1}, m_{2}, \ldots$ ), взаимодействие которых между собой описывается некоторым потенциалом. Это моделирование сводится к отслеживанию движения атомов относительно центра масс, так как сам центр масс движется по инерции. Как только расстояние от ядра некоторого атома $\mathrm{A}_{j}$ до поверхности становится равным $R_{j}$, происходит мгновенное соударение этого атома с поверхностью, которое моделируется с помощью процедуры $C E\left(m_{j},\left\{M_{n, j}\right\},\left\{\mu_{n, j}\right\}, T_{s}, u_{0}\right)$, где $u_{0}<0-z$-компонента скорости атома непосредственно перед соударением. В результате соударения (в предположении, что процедура «оборвется» после конечного числа ударов атома об условные частицы поверхности) $z$-компонента скорости атома становится равной $u_{\perp}>0$ и он «отлетает» от поверхности, а «горизонтальная» компонента скорости атома остается неизменной. После этого возобновляется свободное движение системы атомов с данным потенциалом - до следующего соударения какого-либо атома с поверхностью (или до окончания интегрирования траектории).

В начальный момент времени расстояние от ядра каждого атома до поверхности больше радиуса этого атома, a $z$-компонента скорости центра масс системы атомов отрицательна (молекула приближается к поверхности). Траектория (эволюция системы атомов) представляет собой свободное движение классических материальных точек (с данным потенциалом), которое время от времени прерывается мгновенными соударениями того или иного атома с твердой поверхностью. В свою очередь, каждое соударение есть серия мгновенно следующих друг за другом упругих ударов атома об условные частицы поверхности. Интегрирование траектории прекращается, когда (в соответствии с теми или иными критериями) есть основания полагать, что новых соударений атомов с поверхностью уже не будет. Конечно, эти критерии включают положительность $z$-компоненты скорости центра масс системы атомов. Естественно считать, что в критерии, кроме $z$-компоненты скорости центра масс, входят только $z$-координата центра масс, а также положения и скорости атомов относительно центра масс. «Горизонтальная» компонента скорости центра масс системы атомов на протяжении всей траектории остается неизменной.

Если молекула состоит из одного атома (или иона), то каждая траектория этой молекулы включает, разумеется, единственное соударение, в результате которого $z$-компонента скорости атома меняет знак (с отрицательного на положительный). Отразившись от плоской поверхности, «одинокий» атом уже никогда к ней не вернется.

\section{2. Почти одинаковые траектории}

В рамках такого подхода к моделированию рассеяния молекул на поверхности справедлива следующая очевидная (но очень важная) теорема. Пусть начальные условия двух 
траекторий различаются только «горизонтальными» компонентами $\mathbf{v}_{(1)}$ и $\mathbf{v}_{(2)}$ скорости центра масс системы атомов в начальный момент времени $t=0$, в то время как начальные $z$-компоненты скорости центра масс совпадают, равно как и начальные координаты центра масс и начальные положения и скорости атомов относительно центра масс. Тогда эволюция системы атомов, соответствующая этим траекториям, совершенно одинакова, за исключением разной скорости «сноса» всей системы как целого параллельно поверхности (мы считаем, что для рассматриваемых траекторий совпадают процедуры интегрирования уравнений движения атомов в системе центра масс в промежутках между соударениями атомов с поверхностью, а также процедуры розыгрыша скоростей условных частиц поверхности при соударениях, включая инициализацию датчиков случайных чисел). Иначе говоря, если для первой траектории зависимости положений и скоростей атомов от времени $t$ выражаются функциями

$$
\mathbf{Q}_{j}(t), \mathbf{V}_{j}(t), \quad j=1,2, \ldots
$$

(с той оговоркой, что в момент соударения атома с поверхностью скорость этого атома не определена), то для второй траектории эти зависимости выражаются функциями

$$
\mathbf{Q}_{j}(t)+\mathbf{v}_{(2)} t-\mathbf{v}_{(1)} t, \mathbf{V}_{j}(t)+\mathbf{v}_{(2)}-\mathbf{v}_{(1)}, \quad j=1,2, \ldots
$$

В частности, совпадают конфигурации атомов относительно центра масс в любой момент времени, моменты соударений атомов с поверхностью и атомы, участвующие в этих соударениях, «структура» соударений (последовательность ударов атома об условные частицы поверхности) и моменты прекращения интегрирования траекторий.

Обе траектории характеризуются одинаковыми значениями $W(t)$ внутренней энергии системы атомов в любой момент времени $t$, но разными значениями $E_{(1)}(t)$ и $E_{(2)}(t)$ поступательной энергии системы и разными значениями $H_{(1)}(t)=E_{(1)}(t)+W(t) \quad$ и $H_{(2)}(t)=E_{(2)}(t)+W(t)$ полной энергии (с той оговоркой, что в момент соударения атома с поверхностью все эти энергии не определены). Однако имеют место тождества

$$
E_{(2)}(t)-E_{(2)}(0) \equiv E_{(1)}(t)-E_{(1)}(0)
$$

и, следовательно,

$$
H_{(2)}(t)-E_{(2)}(0) \equiv H_{(1)}(t)-E_{(1)}(0) .
$$

Действительно, «горизонтальные» компоненты скорости центра масс системы атомов для данных траекторий в любой момент времени $t$ равны $\mathbf{v}_{(1)}$ и $\mathbf{v}_{(2)}$, а $z$-компоненты $u(t)$ совпадают. Поэтому

$$
E_{(1)}(t)=\frac{1}{2}\left[u(t)^{2}+\mathbf{v}_{(1)}^{2}\right] \sum_{j} m_{j}, \quad E_{(2)}(t)=\frac{1}{2}\left[u(t)^{2}+\mathbf{v}_{(2)}^{2}\right] \sum_{j} m_{j},
$$

откуда и вытекают указанные выше тождества.

Конечно, независимость динамики рассеяния системы атомов на поверхности от «горизонтальной» компоненты начального полного импульса системы имеет место только в силу того, что «горизонтальная» компонента скорости каждого атома при соударениях с поверхностью не меняется (а также, разумеется, в силу того, что изменение «вертикальной» компоненты не зависит от «горизонтальной» компоненты). Не составляет никакого труда включить тем или иным способом в процедуру составных соударений, описанную в подразделе 3.2, аккомодацию на поверхности «горизонтальной» компоненты импульса атома за счет тангенциальных сил. Однако при этом изменение $\Delta \mathbf{u}_{=}$«горизонтальной» компоненты $\mathbf{u}_{=}$скорости атома при соударении будет неизбежно зависеть от значения $\mathbf{u}_{=}$непосредст- 
венно перед соударением, что приведет к разрушению соответствия между траекториями, о котором шла речь в этом подразделе. Как следствие, мы получим совершенно другой тип моделей рассеяния системы атомов на поверхности. Единственная физически осмысленная ситуация, в которой $\Delta \mathbf{u}_{=}$не зависит от значения $\mathbf{u}_{=}$непосредственно перед соударением, состоит в том, что $\Delta \mathbf{u}_{=}=0$.

Рассмотрим этот вопрос подробнее на примере двух траекторий, о которых шла речь выше. Предположим для определенности, что в рамках первой траектории первым испытывает соударение с поверхностью атом $\mathrm{A}_{1}$. Пусть непосредственно перед соударением (в момент времени $t_{*}$ ) $z$-компонента скорости этого атома равна $u_{0}<0$, а «горизонтальная» компонента равна $\tilde{\mathbf{u}}_{=}$. Допустим, что непосредственно после соударения $z$-компонента скорости атома $\mathrm{A}_{1}$ равна $u_{\perp}>0$, а «горизонтальная» компонента равна $\mathbf{u}_{=}^{*}$ (во многих моделях естественно полагать, что векторы $\mathbf{u}_{=}^{*}$ и $\tilde{\mathbf{u}}_{=}$коллинеарны). Скорости остальных атомов в момент соударения атома $\mathrm{A}_{1}$ с поверхностью равны $\mathbf{V}_{j}\left(t_{*}\right), j=2,3, \ldots$ В рамках второй траектории первым испытывает соударение с поверхностью также атом $\mathrm{A}_{1}$ (в тот же момент времени $t_{*}$ ), причем непосредственно перед соударением $z$-компонента скорости этого атома также равна $u_{0}<0$, а «горизонтальная» компонента равна $\widetilde{\mathbf{u}}_{=}+\mathbf{v}_{(2)}-\mathbf{v}_{(1)}$. Скорости остальных атомов в момент времени $t_{*}$ равны $\mathbf{V}_{j}\left(t_{*}\right)+\mathbf{v}_{(2)}-\mathbf{v}_{(1)}, j=2,3, \ldots$ Непосредственно после соударения $z$-компонента скорости атома $\mathrm{A}_{1}$ также равна $u_{\perp}>0$. Чтобы соответствие между траекториями продолжалось дальше, при временах $t>t_{*}$, «горизонтальная» компонента скорости атома $\mathrm{A}_{1}$ непосредственно после соударения должна быть равна $\mathbf{u}_{=}^{*}+\mathbf{v}_{(2)}-\mathbf{v}_{(1)}$. Но ситуация, когда «горизонтальная» компонента скорости атома при соударении испытывает скачок $\tilde{\mathbf{u}}_{=} \mapsto \mathbf{u}_{=}^{*} \neq \tilde{\mathbf{u}}_{=}$, который остается неизменным $\left(\tilde{\mathbf{u}}_{=}+\mathbf{v}_{(2)}-\mathbf{v}_{(1)} \mapsto \mathbf{u}_{=}^{*}+\mathbf{v}_{(2)}-\mathbf{v}_{(1)}\right)$ при любом векторе $\mathbf{v}_{(2)}-\mathbf{v}_{(1)}$, физически абсолютно нереалистична. В частности, если векторы $\mathbf{u}_{=}^{*} \neq \tilde{\mathbf{u}}_{=}$и $\tilde{\mathbf{u}}_{=}$коллинеарны, а вектор $\mathbf{v}_{(2)}-\mathbf{v}_{(1)}$ им неколлинеарен, то векторы $\mathbf{u}_{=}^{*}+\mathbf{v}_{(2)}-\mathbf{v}_{(1)}$ и $\tilde{\mathbf{u}}_{=}+\mathbf{v}_{(2)}-\mathbf{v}_{(1)}$ неколлинеарны.

\section{5. Рассеяние молекул KI на поверхности графита}

\section{1. Массы и радиусы ионов и потенциал взаимодействия}

Модель диссоциации молекул КІ на поверхности графита, предложенная нами в статьях $[10,11]$, относится к тому классу моделей рассеяния молекул на твердой поверхности, о котором шла речь в разделе 4. Хотя эта модель представлена в работах $[10,11]$ достаточно подробно, мы считаем целесообразным повторить здесь описание ее основных характеристик, потому что при этом будет введен ряд понятий, необходимых для последующего изложения.

В модели рассматривается движение ионов $\mathrm{K}^{+}$и $Г$, хотя молекула иодида калия характеризуется смешанным типом диссоциации и может распадаться как на ионы $\left(\mathrm{K}^{+}\right.$и Г $)$, так и на нейтральные атомы (K и I) [40]. В дальнейшем в контексте раздела 4 катиону калия $\mathrm{K}^{+}$будет соответствовать нижний индекс 1 , а аниону йода $\mathrm{I}^{-}-$индекс 2 . Массы ионов $m_{1}=39.0983$ Да и $m_{2}=126.90447$ Да отвечают атомным весам калия и йода. Значения радиусов ионов полагались равными кристаллическим радиусам $R_{1}=1.33 \AA$ и $R_{2}=2.19 \AA$ [41]. В качестве потенциала взаимодействия в системе $\mathrm{K}^{+}-\mathrm{I}^{-}$был взят потенциал в широко применяемой и достаточно гибкой усеченной форме Риттнера [41-43]: в атомной системе единиц 


$$
V(r)=A e^{-r / \rho}-\frac{1}{r}-\frac{\alpha_{1}+\alpha_{2}}{2 r^{4}}-\frac{C}{r^{6}},
$$

где $r$ - расстояние между ядрами ионов. Здесь $\alpha_{1}=5.5$ Бор $^{3}$ и $\alpha_{2}=60.2$ Бор $^{3}-$ поляризуемости ионов $\mathrm{K}^{+}$и Г соответственно [44], а значения калибровочного множителя $A$, параметра мягкости/жесткости $\rho$ и дисперсионной постоянной $C$ в атомной системе единиц мы полагали равными $A=286.799, \rho=0.639272, C=138$. Значение $C$ приведено в статье [44]. Значения $A$ и $\rho$ были выбраны исходя из того требования, чтобы при указанных значениях $\alpha_{1}, \alpha_{2}$ и $C$ потенциал $V(r)$ имел при $r=R_{m}=5.76$ Бор минимум $-\varepsilon=-0.1722$ Хартри. Эти экспериментальные значения положения $R_{m}$ и глубины $\varepsilon$ потенциальной ямы для ионного потенциала взаимодействия в молекуле КI также приведены в статье [44].

Отметим, что подробная аннотированная библиография работ по потенциалам взаимодействия во всех галогенидах щелочных металлов представлена в отчете [45].

При соударениях ионов с поверхностью изменяется полная внутренняя энергия пары ионов

$$
W=\frac{1}{2} m_{\mathrm{red}} \dot{\mathbf{r}}^{2}+V(r),
$$

которая в промежутках между соударениями остается постоянной. Здесь $\mathbf{r}-$ вектор, соединяющий ядра ионов $(r=|\mathbf{r}|)$, а $m_{\text {red }}=m_{1} m_{2} /\left(m_{1}+m_{2}\right)$ - их приведенная масса. В частности, может измениться знак энергии $W-$ с отрицательного на положительный (что отвечает диссоциации) или с положительного на отрицательный (что отвечает рекомбинации ионов). Отметим, что кулоновский член $-1 / r$ в потенциале $V(r)$ исключает образование центробежного барьера, так что система ионов с положительной полной внутренней энергией $W$ всегда является несвязанной.

\section{2. Интегрирование уравнений движения}

Движение системы ионов в промежутках между соударениями ионов с поверхностью графита - это свободное движение пары материальных точек с массами $m_{1}$ и $m_{2}$ и потенциалом взаимодействия $V(r)$. Трехмерная задача о расчете движения пары таких материальных точек в пустом пространстве стандартным образом сводится к двумерной задаче о движении точки массы $m_{\text {red }}$ в центральном поле с тем же потенциалом на плоскости, ортогональной сохраняющемуся вектору момента количества движения $\mathbf{L}=m_{\mathrm{red}}[\mathbf{r}, \dot{\mathbf{r}}]$. Численное интегрирование соответствующих ньютоновых уравнений движения в работе [11] мы проводили методом Адамса-Башфорта шестого порядка, при этом первые пять шагов интегрирования (после розыгрыша начальных условий или после очередного соударения одного из ионов с поверхностью графита) выполнялись методом Рунге-Кутта четвертого порядка. Точный момент каждого соударения определялся с помощью серии пробных одношаговых интегрирований уравнений движения назад методом Рунге-Кутта четвертого порядка. Шаг интегрирования уравнений движения мы полагали равным $h=5$ a. е. Такое значение $h$ обеспечивало сохранение полной внутренней энергии $W$ пары ионов и момента количества движения $L$ в промежутках между соударениями ионов с поверхностью графита до $\approx 10$ значащих цифр.

\section{3. Массы условных частиц графита}

Для описания рассеяния системы ионов $\mathrm{K}^{+}-\mathrm{I}^{-}$на поверхности графита при помощи «модели составных соударений» раздела 3 необходимо для $j$-ого иона $(j=1,2)$ задать по- 
следовательность «столкновительных» масс $\left\{M_{n, j}\right\}_{n \geq 1}$ и последовательность «максвелловских» масс $\left\{\mu_{n, j}\right\}_{n \geq 1}$. В модели $[10,11]$ все «максвелловские» массы мы полагали равными массе $m_{\mathrm{C}}$ атома углерода:

$$
\mu_{n, j}=m_{\mathrm{C}}=12.0107 \text { Да }
$$

для всех $n \geq 1$ и обоих $j=1,2$. «Столкновительные» же массы $M_{n, j}$ полагались пропорциональными номеру $n$ :

$$
M_{n, j}=n M_{1, j}
$$

(мы считали, что условная частица графита, участвующая в $n$-ом упругом ударе иона о поверхность в рамках данного соударения, «объединяет» $n$ слоев графита, или базисных плоскостей, параллельных поверхности). Массы $M_{1,1}$ и $M_{1,2}$ определялись следующим образом.

Каждый слой графита (параллельный его поверхности $z=0$ ) представляет собой бесконечную сетку гексагонально расположенных атомов углерода, при этом сторона шестиугольников (длина C-C связи) равна $\ell=1.418 \AA$ [46]. Если каждая вершина гексагональной сетки является центром круга радиуса $a \leq \ell / 2$, представляющего «сечение» атома углерода (предположение $a \leq \ell / 2$ в принципе допустимо, так как атомный радиус атома углерода составляет $0.77 \AA$ [47], а $\ell / 2=0.709 \AA$ ), то «доля плоскости», занимаемая этими кругами, составляет $\lambda=3^{-3 / 2} 4 \pi a^{2} / \ell^{2}$. Действительно, площадь каждого шестиугольника равна $S_{6}=3^{3 / 2} \ell^{2} / 2$, а суммарная площадь шести секторов кругов, содержащихся в данном шестиугольнике, равна $S_{0}=6\left(\pi a^{2} / 3\right)=2 \pi a^{2}$, и для $\lambda=S_{0} / S_{6}$ мы получаем указанное выше значение. Ион радиуса $R_{j}$ «накрывает» в среднем $\lambda \pi R_{j}^{2} /\left(\pi a^{2}\right)=3^{-3 / 2} 4 \pi R_{j}^{2} / \ell^{2}$ атомов углерода, и их суммарная масса и есть $M_{1, j}$. Итак,

$$
M_{1, j}=3^{-3 / 2} 4 \pi R_{j}^{2} m_{\mathrm{C}} / \ell^{2},
$$

где, как и раньше, $m_{\mathrm{C}}$ - масса атома углерода (отметим, что $M_{1, j}$ не зависит от $a$ ). Для ионов $\mathrm{K}^{+}$и Г вычисления дают $M_{1,1}=25.55331$ Да и $M_{1,2}=69.28386$ Да.

Согласно подразделу 3.4 при таких «столкновительных» и «максвелловских» массах условных частиц графита любое соударение ионов с поверхностью включает только конечное число упругих ударов.

\section{4. Розыгрыш начальных условий}

Чтобы закончить описание нашей модели рассеяния молекул КI на поверхности графита, необходимо еще указать процедуру розыгрыша начальных условий и критерии прекращения интегрирования траектории. Пусть в эксперименте поступательная энергия молекул KI в пучке равна $E$, а угол между осью пучка и поверхностью графита (угол падения пучка) равен $\gamma\left(0<\gamma \leq 90^{\circ}\right)$. Для моделирования взаимодействия молекул КІ с поверхностью графита в этом случае мы предполагали, что ось пучка лежит в координатной плоскости $O x z$, т. е. начальная скорость центра масс молекулы равна $\left(v_{E} \cos \gamma, 0,-v_{E} \sin \gamma\right)$, где $v_{E}=\left[2 E /\left(m_{1}+m_{2}\right)\right]^{1 / 2}-$ абсолютная величина скорости. «Горизонтальная» компонента начальной скорости центра масс молекулы равна $\left(v_{E} \cos \gamma, 0\right)$, а «вертикальная»компонента ( $z$-компонента) равна $-v_{E} \sin \gamma$. Соответственно «горизонтальная составляющая» началь- 
ной поступательной энергии $E$ молекул KI равна $E \cos ^{2} \gamma$, а «вертикальная составляющая» равна $E \sin ^{2} \gamma$.

Начальное расстояние между центром масс молекулы КІ и поверхностью графита мы в статье [11] всегда полагали равным 15 Бор. Расстояние между ядрами ионов в молекуле KI в начале траектории, начальная ориентация оси молекулы и начальные скорости ионов относительно центра масс молекулы разыгрывались стандартным образом исходя из колебательной температуры $T_{v}=600 \mathrm{~K}$ и вращательной температуры $T_{r}=70 \mathrm{~K}$ молекул в пучке. Эти значения $T_{v}$ и $T_{r}$ адекватно отражают экспериментальные условия $[9,11]$.

\section{5. Критерии прекращения интегрирования траектории}

Мы в работе [11] прекращали интегрирование траектории с отнесением ее к категории «недиссоциативных» (отвечающих неупругому рассеянию), если после данного шага интегрирования оказывались выполненными следующие три условия.

a) Полная внутренняя энергия $W$ (9) пары ионов отрицательна (т. е. ионы, как и в начале траектории, образуют связанную систему).

б) $z$-компонента скорости центра масс пары ионов положительна.

в) $z$-координата $Z$ центра масс пары ионов настолько велика $\left(Z>Z_{o}\right)$, что новые соударения ионов с поверхностью графита при дальнейшем движении ионов исключены. Прежде чем привести формулу для $Z_{o}$, которую мы использовали, оценим сверху максимальное расстояние $r_{\max }$ между ядрами ионов, возможное для данного значения $W<0$ полной внутренней энергии. Из выражений (8) и (9) мы получаем (в атомной системе единиц) неравенство

$$
-\frac{1}{r}\left(1+\frac{\alpha_{1}+\alpha_{2}}{2 r^{3}}+\frac{C}{r^{5}}\right)=W-\frac{1}{2} m_{\mathrm{red}} \dot{\mathbf{r}}^{2}-A e^{-r / \rho}<W,
$$

справедливое для любого межъядерного расстояния $r$, возможного для данного значения $W<0$. Следовательно,

$$
r_{\max }<-\frac{1}{W}\left(1+\frac{\alpha_{1}+\alpha_{2}}{2 r_{\max }^{3}}+\frac{C}{r_{\max }^{5}}\right) \leq q(r)=-\frac{1}{W}\left(1+\frac{\alpha_{1}+\alpha_{2}}{2 r^{3}}+\frac{C}{r^{5}}\right)
$$

(в последней формуле $r$ - текущее расстояние между ядрами ионов). Кроме того, если межъядерное расстояние в молекуле КІ равно $r$, то расстояние между центром масс молекулы и ядром иона $\mathrm{K}^{+}$есть $m_{2} r /\left(m_{1}+m_{2}\right)$, а расстояние между центром масс молекулы и ядром иона $\mathrm{I}^{-}$есть $m_{1} r /\left(m_{1}+m_{2}\right)$. Поэтому для $Z_{o}$ мы использовали выражение

$$
Z_{o}=\max \left(\frac{m_{2} q(r)}{m_{1}+m_{2}}+R_{1}, \frac{m_{1} q(r)}{m_{1}+m_{2}}+R_{2}\right) .
$$

С другой стороны, мы прекращали интегрирование траектории с отнесением ее к категории «диссоциативных», если после данного шага интегрирования оказывались выполненными следующие четыре условия.

a) Полная внутренняя энергия $W$ (9) пары ионов положительна и, кроме того, значение $\frac{1}{2} m_{\mathrm{red}} \dot{\mathbf{r}}^{2}-1 / r$ (в атомной системе единиц) положительно.

б) Расстояние между ядрами ионов достаточно велико, точнее, больше некоторой константы, которую мы полагали равной 200 Бор, и продолжает увеличиваться: $\dot{r}=(\mathbf{r}, \dot{\mathbf{r}}) / r>0$.

в) $z$-компонента скорости центра масс пары ионов положительна. 
При выполнении этих первых трех условий мы вычисляли скорости катиона калия $\mathbf{V}_{1}$ и аниона йода $\mathbf{V}_{2}$ «на бесконечности» (т. е. после разлета ионов на бесконечное расстояние) в предположении, что их дальнейшее движение происходит в пустом пространстве в поле кулоновского потенциала $-1 / r$. При этом мы пользовались алгоритмом, изложенным в конце раздела 3 отчета [48]. Четвертое условие состоит в следующем.

г) $z$-компоненты обеих скоростей $\mathbf{V}_{1}$ и $\mathbf{V}_{2}$ положительны.

Направление скорости $\mathbf{V}_{j}(j=1,2)$ характеризуется углом $\vartheta_{j}$ между вектором $\mathbf{V}_{j}$ и плоскостью $O x z$ (в которой лежит ось пучка молекул $\mathrm{KI}$ ) и углом $\varphi_{j}$ между осью $O x$ и проекцией вектора $\mathbf{V}_{j}$ на плоскость $O x z$. Таким образом,

$$
\mathbf{V}_{j}=\left(\cos \vartheta_{j} \cos \varphi_{j}, \pm \sin \vartheta_{j}, \cos \vartheta_{j} \sin \varphi_{j}\right)\left|\mathbf{V}_{j}\right|,
$$

где $0 \leq \vartheta_{j}<90^{\circ}$ и $0<\varphi_{j}<180^{\circ}$. В соответствии с дизайном экспериментальной установки мы считали, что ось детектора лежит в плоскости $O x z$, и детектор может зарегистрировать только те ионы, для которых $\vartheta_{j} \leq 2^{\circ}$ (т. е. скорость иона очень мало отклоняется от плоскости $O x z$ ) и $\varphi_{j}<90^{\circ}$. В эксперименте рассеянные ионы $\mathrm{K}^{+}$и $\mathrm{I}^{-}$регистрировались детектором с установленным на его входе энергоанализатором, причем входная диафрагма анализатора диаметром 1.5 мм располагалась на расстоянии 20 мм от поверхности мишени (пластинки графита или алмаза). Такие параметры отвечают предельному углу

$$
\vartheta_{\max }=\operatorname{arctg} \frac{1.5 / 2}{20} \approx 2.15^{\circ} .
$$

\section{6. Обзор вычислений}

В статье [11] приведены результаты расчетов диссоциативного и недиссоциативного рассеяния молекул KI на поверхности графита в рамках описанной модели для поступательных энергий $E$ молекул в пучке от 4 до 14 эВ, для углов $\gamma$ между осью пучка и поверхностью графита (углов падения пучка) от $5^{\circ}$ до $85^{\circ}$ и для двух значений температуры поверхности: $T_{s}=298 \mathrm{~K}\left(\approx 25^{\circ} \mathrm{C}\right)$ и $T_{s}=523 \mathrm{~K}\left(\approx 250^{\circ} \mathrm{C}\right)$. Всего было рассмотрено 250 троек параметров $\left(E, \gamma, T_{s}\right)$, по 125 троек для каждого из двух значений $T_{s}$, и для каждой тройки интегрировалось $N=10^{7}$ траекторий.

Максимальное число упругих ударов иона об условные частицы графита за один акт соударения иона с поверхностью, которое мы наблюдали (по всем траекториям для всех значений $E, \gamma$ и $T_{s}$, взятых вместе), было равно 7 - как для иона $\mathrm{K}^{+}$, так и для иона $Г$.

Любой траектории, на протяжении которой произошло $k \geq 1$ соударений ионов с поверхностью графита, можно сопоставить последовательность натуральных чисел $\left(\xi_{1}, \xi_{2}, \ldots, \xi_{k}, \eta\right)$, где $\xi_{i}=1\left(\xi_{i}=2\right)$, если в $i$-ом соударении участвовал ион $\mathrm{K}^{+}$(соответственно ион $\left.\mathrm{I}^{-}\right)$, а $\eta=3$ ( $\left.\eta=4\right)$, если рассматриваемая траектория является «недиссоциативной» (соответственно «диссоциативной»). Эта последовательность в [11] названа типом траектории.

В работе [11] изучались следующие характеристики взаимодействия молекул КІ с поверхностью графита, определенные для данных значений $E, \gamma$ и $T_{s}$ :

а) число $N_{d}$ «диссоциативных» траекторий и вероятность диссоциации $P=N_{d} / N$;

б) число «диссоциативных» траекторий с $\vartheta_{j} \leq 2^{\circ}$, а также число «диссоциативных» траекторий с $\vartheta_{j} \leq 2^{\circ}$ и $\varphi_{j}<90^{\circ}(j=1,2)$; 
в) распределение ионов по углу $\vartheta_{j}$ и распределение ионов с $\vartheta_{j} \leq 2^{\circ}$ по углу $\varphi_{j}$ $(j=1,2)$;

г) статистика энергий $G_{1}$ и $G_{2}$ образовавшихся свободных ионов $\mathrm{K}^{+}$и $\mathrm{I}^{-}$;

д) статистика числа упругих ударов данного иона об условные частицы графита в рамках одного соударения иона с поверхностью (по всем соударениям, имевшим место в $10^{7}$ проинтегрированных траекторий);

е) статистика числа соударений данного иона с поверхностью графита в пределах одной траектории (по всему ансамблю из $10^{7}$ проинтегрированных траекторий, а также отдельно по «диссоциативным» и «недиссоциативным» траекториям);

ж) статистика типов траекторий.

В частности, в статье [11] рассматривалась зависимость этих характеристик от значений $E, \gamma$ и $T_{s}$. Согласно сказанному в подразделе 4.2 характеристики а), д), е) и ж) зависят только от температуры поверхности графита $T_{s}$ и от «вертикальной составляющей» $E \sin ^{2} \gamma$ начальной поступательной энергии $E$ молекул KI, но не зависят от «горизонтальной составляющей» $E \cos ^{2} \gamma$. В работе [11] мы отмечали, что соответствующие величины определяются (при фиксированной температуре $T_{s}$ ) почти исключительно «вертикальной составляющей» $E \sin ^{2} \gamma$ поступательной энергии молекул KI; на самом деле слово «почти» здесь излишнее. Статистика величины $G_{1}+G_{2}-E$ согласно подразделу 4.2 тоже зависит только от $E \sin ^{2} \gamma$ и $T_{s}$, потому что для «диссоциативных» траекторий сумма $G_{1}+G_{2}$ равна, очевидно, конечной полной энергии $H$ системы ионов.

«Доля» начальной поступательной энергии $E$ молекул KI, приходящаяся на $j$-ый ион $(j=1,2)$, равна $m_{j} E /\left(m_{1}+m_{2}\right)$. Интересно, что среднее значение разности

$$
G_{j}-\frac{m_{j}}{m_{1}+m_{2}} E
$$

для каждого иона по всем «диссоциативным» траекториям также зависит только от $E \sin ^{2} \gamma$ и $T_{s}$ (при условии, что число $N_{d}$ «диссоциативных» траекторий достаточно велико). Подчеркнем, что если независимость от $E \cos ^{2} \gamma$ характеристик а), д)-ж) выше и статистики величины $G_{1}+G_{2}-E$ проявляется (при надлежащей организации вычислений) уже «на уровне» отдельных траекторий и выполняется точно, то независимость от $E \cos ^{2} \gamma$ среднего значения разности (15) имеет место лишь приближенно (тем точнее, чем больше $N_{d}$ ).

Для доказательства того, что среднее значение разности (15) зависит только от $E \sin ^{2} \gamma$ и $T_{s}$, рассмотрим «диссоциативную» траекторию, у которой $z$-компонента начальной скорости центра масс молекулы KI равна $u<0$, а «горизонтальная» компонента начальной скорости центра масс молекулы равна $\mathbf{v}$, так что для этой траектории

$$
E=\frac{1}{2}\left(m_{1}+m_{2}\right)\left(u^{2}+\mathbf{v}^{2}\right), \quad \gamma=\operatorname{arctg} \frac{|u|}{|\mathbf{v}|}, \quad \frac{m_{j}}{m_{1}+m_{2}} E=\frac{1}{2} m_{j}\left(u^{2}+\mathbf{v}^{2}\right) .
$$

Обозначим $z$-компоненту конечной скорости $j$-ого иона для этой траектории через $a_{j}>0$, a «горизонтальную» компоненту конечной скорости $j$-ого иона через $\mathbf{b}_{j}+\mathbf{v}$. Тогда для рассматриваемой траектории

$$
G_{j}=\frac{1}{2} m_{j}\left(a_{j}^{2}+\mathbf{b}_{j}^{2}+2 \mathbf{b}_{j} \cdot \mathbf{v}+\mathbf{v}^{2}\right),
$$


где $\mathbf{b}_{j} \cdot \mathbf{v}$ - скалярное произведение векторов $\mathbf{b}_{j}$ и $\mathbf{v}$. Поэтому

$$
G_{j}-\frac{m_{j}}{m_{1}+m_{2}} E=\frac{1}{2} m_{j}\left(a_{j}^{2}+\mathbf{b}_{j}^{2}+2 \mathbf{b}_{j} \cdot \mathbf{v}-u^{2}\right) .
$$

Отметим, что $m_{1} \mathbf{b}_{1}+m_{2} \mathbf{b}_{2}=0$ в силу сохранения «Горизонтальной» компоненты скорости центра масс системы ионов.

Согласно рассуждениям подраздела 4.2 можно установить (при фиксированной температуре $T_{s}$ ) взаимно-однозначное соответствие между «диссоциативными» траекториями, характеризующимися указанными в (16) значениями $E$ и $\gamma$, и «диссоциативными» траекториями, у которых начальная скорость центра масс молекулы KI равна $u$ и направлена ортогонально поверхности графита. При таком соответствии рассматриваемой траектории отвечает траектория, у которой $z$-компонента конечной скорости $j$-ого иона также равна $a_{j}$, a «горизонтальная» компонента конечной скорости $j$-ого иона равна $\mathbf{b}_{j}$. Обозначая усреднение по всем «диссоциативным» траекториям (с данными значениями $E, \gamma$ и $T_{s}$ ) угловыми скобками, мы видим, что в силу симметрии $\left\langle\mathbf{b}_{j}\right\rangle=0$ (если $\gamma=90^{\circ}$, то все «горизонтальные» направления равноправны). Значит,

$$
\left\langle G_{j}-\frac{m_{j}}{m_{1}+m_{2}} E\right\rangle=\frac{1}{2} m_{j}\left(\left\langle a_{j}^{2}\right\rangle+\left\langle\mathbf{b}_{j}^{2}\right\rangle-u^{2}\right)
$$

зависит только от

$$
u=-\left(\frac{2 E \sin ^{2} \gamma}{m_{1}+m_{2}}\right)^{1 / 2}
$$

и не зависит от $\mathbf{v}$.

\section{6. Рассеяние молекул KI на других твердых поверхностях}

\section{1. Задание поверхности «столкновительными» и «максвелловскими» массами}

В импульсной модели рассеяния молекул КІ на поверхности графита, предложенной в работах $[10,11]$ и кратко изложенной в разделе 5 выше, мы попытались учесть специфику поверхности за счет выбора (10)-(12) «столкновительных» и «максвелловских» масс, характеризующих взаимодействие ионов $\mathrm{K}^{+}$и $\mathrm{I}^{-}$с поверхностью. Оставшаяся часть настоящей статьи посвящена обсуждению моделей рассеяния молекул КI на твердой поверхности, которые отличаются от рассмотренной модели только другим выбором «столкновительных» $M_{n, j}$ и «максвелловских» $\mu_{n, j}$ масс $(n \geq 1$ и $j=1,2)$ - при полном сохранении всех остальных параметров (включая колебательную $T_{v}$ и вращательную $T_{r}$ температуры молекул). При этом мы будем рассматривать только такие последовательности масс $\left\{M_{n, j}\right\}_{n \geq 1}$ и $\left\{\mu_{n, j}\right\}_{n \geq 1}$, для которых теоремы подраздела 3.4 гарантируют, что всякое соударение ионов с поверхностью включает только конечное число упругих ударов.

При любом выборе «столкновительных» $M_{n, j}$ и «максвелловских» $\mu_{n, j}$ масс вероятность диссоциации $P$, статистика величины $G_{1}+G_{2}-E$ по «диссоциативным» траекториям, статистика числа упругих ударов данного иона об условные частицы поверхности в рамках одного соударения иона с поверхностью, статистика числа соударений данного иона с поверхностью в пределах одной траектории и статистика типов траекторий (см. подраз- 
дел 5.6) определяются только температурой поверхности $T_{s}$ и «вертикальной составляющей» $E \sin ^{2} \gamma$ начальной поступательной энергии $E$ молекул KI и не зависят от «горизонтальной составляющей» $E \cos ^{2} \gamma$. То же справедливо и для среднего значения разности (15) для каждого иона по «диссоциативным» траекториям (если число $N_{d}$ таких траекторий достаточно велико).

В разделе 7 статьи [11] было высказано предположение, что для моделирования рассеяния двухатомных молекул с ионной связью на поверхности алмаза можно все «максвелловские» массы по-прежнему положить равными массе $m_{\mathrm{C}}$ атома углерода (т. е. оставить в силе (10)), а все «столкновительные» массы взять бесконечными и таким образом учесть твердость алмаза:

$$
M_{n, j}=\infty
$$

для всех $n \geq 1$ и обоих $j=1,2$.

Мы провели расчет диссоциативного и недиссоциативного рассеяния молекул КI на поверхности для «столкновительных» и «максвелловских» масс, заданных равенствами (18) и (10). Были использованы те же два значения температуры поверхности, $T_{s}=298 \mathrm{~K}$ и $T_{s}=523 \mathrm{~K}$. Угол $\gamma$ между осью пучка и поверхностью варьировался от $5^{\circ}$ до $90^{\circ}$, а поступательная энергия $E$ молекул в пучке - от $10^{-4}$ эВ (т. е. 0.1 мэВ) до 14 эВ. Для каждой тройки параметров $\left(E, \gamma, T_{s}\right)$ с $E \geq 1$ мэВ интегрировалось $N=10^{7}$ траекторий, а при $E=0.1$ мэВ $-4 \times 10^{6}$ траекторий.

Кроме того, мы провели некоторый дополнительный (по отношению к [11]) расчет рассеяния молекул КІ на поверхности для масс $M_{n, j}$ и $\mu_{n, j}$, заданных равенствами (10)(12).

На рис. 1 в качестве примера показаны зависимости вероятности диссоциации $P=N_{d} / N\left(N_{d}\right.$ - число «диссоциативных» траекторий) от «вертикальной составляющей» $E \sin ^{2} \gamma$ энергии $E$ для обоих значений температуры поверхности $T_{s}$ и для двух вариантов выбора масс $M_{n, j}$ и $\mu_{n, j}$ - значений (10)-(12), использованных в работах $[10,11]$, и значений (10), (18), предложенных в разделе 7 статьи [11]. Как видно из этого рисунка, вероятность диссоциации зависит от температуры $T_{s}$ не очень сильно (что противоречит экспериментальным данным по рассеянию KI как на пиролитическом графите $[9,11]$, так и на алмазе).

В качестве другого примера рисунок на англоязычной аннотационной странице в начале статьи показывает зависимости числа различных типов траекторий (см. подраздел 5.6) от «вертикальной составляющей» $E \sin ^{2} \gamma$ энергии $E$ для обоих значений температуры поверхности $T_{s}$ и для двух вариантов выбора масс $M_{n, j}$ и $\mu_{n, j}-$ значений (10)-(12) и значений (10), (18). Конечно, малые «осцилляции» на кривых, изображенных на этом рисунке, вызваны лишь статистическими погрешностями. Для масс (10)-(12) число типов траекторий при переходе от $T_{s}=298 \mathrm{~K}$ к $T_{s}=523 \mathrm{~K}$ всегда заметно уменьшается. Для масс (10), (18) число типов траекторий почти не зависит от температуры $T_{s}$, но для $E \sin ^{2} \gamma \geq 6$ эВ при переходе от $T_{s}=298 \mathrm{~K}$ к $T_{s}=523 \mathrm{~K}$ возрастает.

Для любых фиксированных значений энергии $E \sin ^{2} \gamma$ (в интервале от 50 мэВ до 14 эВ) и температуры $T_{s}$ (равной $298 \mathrm{~K}$ или $523 \mathrm{~K}$ ) при переходе от «столкновительных» и «максвелловских» масс (10)-(12) к массам (10), (18) вероятность диссоциации $P$ увеличива- 
ется, в крайнем случае остается неизменной и равной нулю (см. рис. 1). С другой стороны, такие характеристики, как среднее и максимальное значения числа упругих ударов данного иона об условные частицы поверхности в рамках одного соударения иона с поверхностью (как для иона $\mathrm{K}^{+}$, так и для иона $\mathrm{I}^{-}$), среднее число соударений данного иона с поверхностью в пределах одной траектории (как для иона $\mathrm{K}^{+}$, так и для иона $\mathrm{I}^{-}$), среднее и максимальное значения числа соударений ионов с поверхностью в пределах одной траектории, среднее и максимальное значения числа соударений ионов с поверхностью в пределах одной «недиссоциативной» траектории (а также среднее и максимальное значения числа соударений ионов с поверхностью в пределах одной «диссоциативной» траектории - при $E \sin ^{2} \gamma \geq 4.4$ эВ), число различных типов траекторий (см. рисунок на англоязычной странице в начале статьи), число различных типов «недиссоциативных» траекторий (а также число различных типов «диссоциативных» траекторий - при $E \sin ^{2} \gamma \geq 5$ эВ), уменьшаются (в очень редких случаях остаются неизменными).

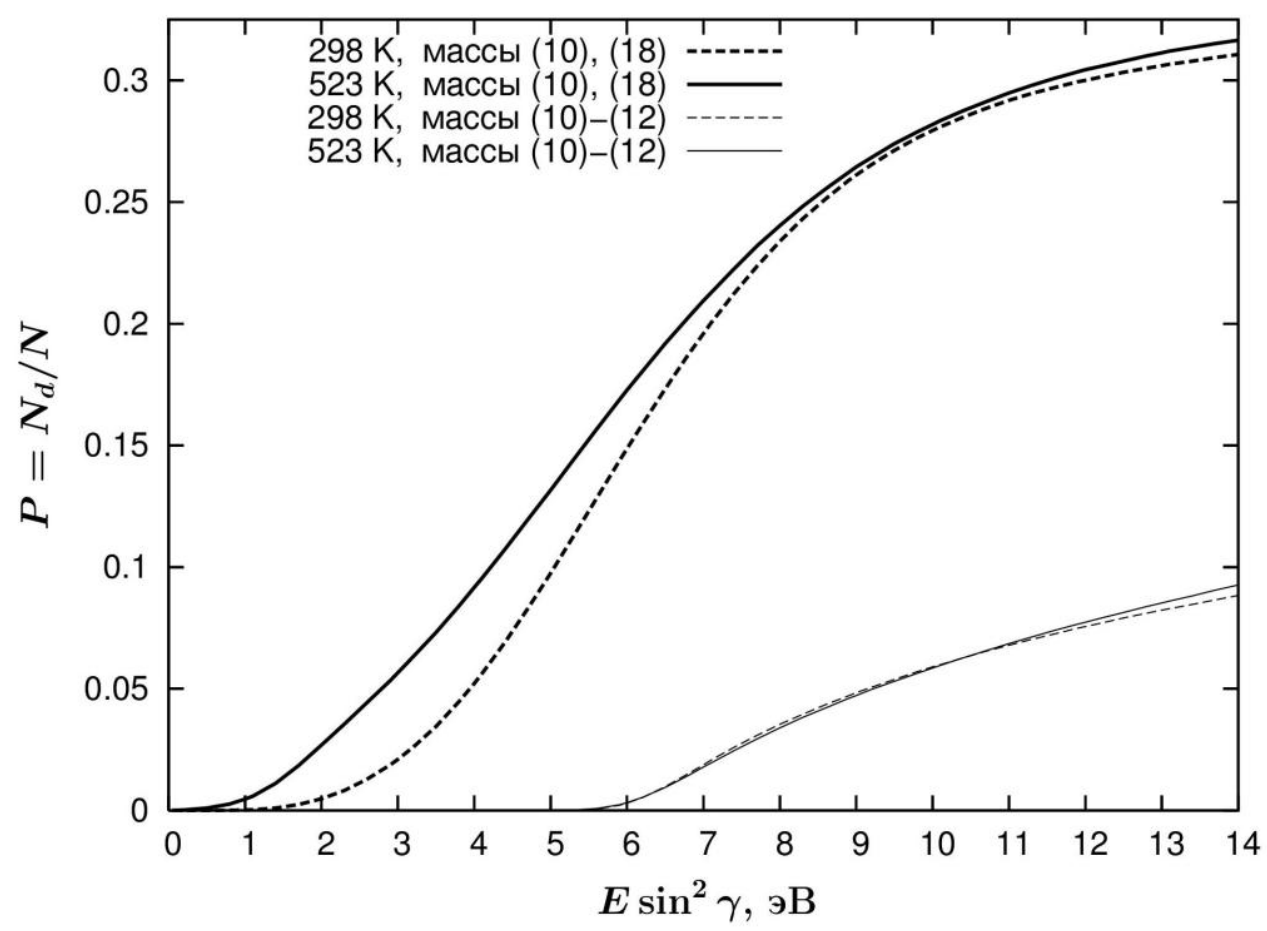

Рис. 1. Зависимости вероятности диссоциации $P$ от «вертикальной составляющей» энергии молекул в пучке для двух значений температуры поверхности $T_{s}$ и

для двух вариантов выбора «столкновительных» и «максвелловских» масс

В случае выбора (10)-(12) «столкновительных» и «максвелловских» масс (т. е. в рамках модели рассеяния молекул КІ на поверхности графита) минимальное значение $E \sin ^{2} \gamma$ в наших расчетах, при котором мы встретили «диссоциативные» траектории, было равно 4.4 эВ при $T_{s}=298 \mathrm{~K}$ и 1.7 эВ при $T_{s}=523 \mathrm{~K}$. В случае выбора (10), (18) «столкновительных» и «максвелловских» масс минимальное значение $E \sin ^{2} \gamma$, при котором наблюдались «диссоциативные» траектории, при $T_{s}=298 \mathrm{~K}$ было равно 100 мэВ. С другой стороны, при $T_{s}=523 \mathrm{~K}$ даже при $E=0.1$ мэВ и $\gamma=90^{\circ}$ число $N_{d}$ «диссоциативных» траекторий получилось равным 44 (на $N=4 \times 10^{6}$ всех траекторий). Для $E=1,10$ и 50 мэВ и $\gamma=90^{\circ}$ мы наблюдали 88, 152 и 434 «диссоциативные» траектории соответственно при общем числе тра- 
екторий, равном $10^{7}$. Конечно, то, что при $E=0.1$ мэВ и $\gamma=90^{\circ}$ вероятность диссоциации $P=1.1 \times 10^{-5}$ оказалась выше, чем при $E=1$ мэВ и $\gamma=90^{\circ}\left(P=8.8 \times 10^{-6}\right)$, вызвано лишь очень малой статистикой.

\section{2. Первый удар молекулы о поверхность}

Эти расчеты рассеяния молекул КІ на поверхности для масс $M_{n, j}$ и $\mu_{n, j}$, заданных равенствами (10), (18), при $T_{s}=523 \mathrm{~K}$ и очень малых $E \sin ^{2} \gamma$ позволили выявить одну неточность в работе [11], связанную с вопросом о том, какой из двух ионов $\mathrm{K}^{+}$и $\mathrm{I}^{-}$чаще ударяет о поверхность первым (и насколько чаще). Для удобства читателя воспроизведем здесь (с незначительными изменениями) соответствующий абзац и рисунок на стр. 85 статьи [11].

"Приближаясь к поверхности графита, молекула КІ ударяет по поверхности ионом $\mathrm{K}^{+}$ (ионом $\mathrm{I}^{-}$), если в обозначениях рис. 2 (в [11] это рис. 8) в момент соударения перпендикуляр, опущенный из ядра иона $\mathrm{I}^{-}$на поверхность, лежит в телесном угле $\omega$ (соответственно в телесном угле $\Omega)$. Величины телесных углов $\Omega$ и $\omega$ равны $2 \pi(1+\cos \beta)$ и $2 \pi(1-\cos \beta)$ соответственно (см. рис. 2), где $\cos \beta=\left(R_{2}-R_{1}\right) / r=\Delta R / r$, и отношение этих величин есть $(r+\Delta R) /(r-\Delta R)$. С точки зрения вращений и колебаний молекулы ее ориентация и фаза колебаний в момент первого соударения с поверхностью графита полностью случайны. Поэтому можно ожидать, что отношение $\zeta$ числа траекторий, для которых первое соударение ионов с поверхностью - это соударение иона Г, к числу траекторий, для которых первым соударением является соударение иона $\mathrm{K}^{+} \mathrm{c}$ поверхностью, будет достаточно близко к среднему значению (равному 1.7805) отношения $(r+\Delta R) /(r-\Delta R)$ по фазе колебаний молекулы KI для температур $T_{v}=600 \mathrm{~K}$ и $T_{r}=70 \mathrm{~K}$. Вычисления подтверждают это предположение. Для любой тройки параметров $\left(E, \gamma, T_{s}\right)$ отношение $\zeta$ лежит между 1.76809 и 1.78331, и в подавляющем числе случаев $\zeta$ очень близко к $1.7805 . "$

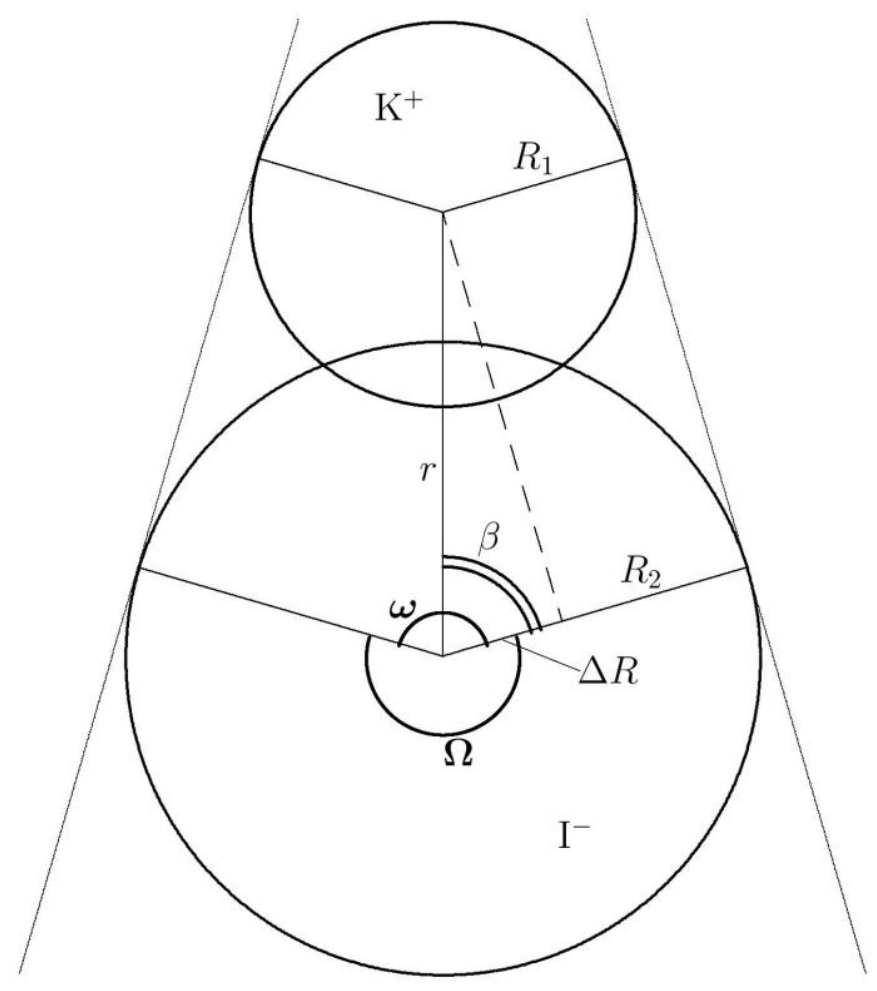

Рис. 2. Молекула KI перед первым соударением с поверхностью 
В этом рассуждении в соответствии с темой работы [11] говорится о графите, но очевидно, что отношение $\zeta$ на самом деле определяется только «вертикальной составляющей» $E \sin ^{2} \gamma$ энергии $E$ и не зависит (при условии достаточной статистики) ни от «горизонтальной составляющей» $E \cos ^{2} \gamma$, ни от температуры поверхности $T_{s}$, ни от масс $M_{n, j}$ и $\mu_{n, j}$. В наших новых расчетах как с массами (10)-(12), так и с массами (10), (18) отношение $\zeta$ при $0.1 \leq E \sin ^{2} \gamma \leq 14$ эВ лежало между 1.77723 и 1.78351 для обоих значений температуры $T_{s}$.

С другой стороны, при $E=50$ мэВ и $\gamma=90^{\circ}$ отношение $\zeta$ в наших новых расчетах было заключено между 1.77608 и 1.77647 (небольшая зависимость $\zeta$ от «столкновительных» и «максвелловских» масс и от температуры поверхности связана со статистическими погрешностями), а при дальнейшем уменьшении $E \sin ^{2} \gamma$ это отношение быстро падало. Для масс $M_{n, j}$ и $\mu_{n, j}$, задаваемых равенствами (10), (18), и $T_{s}=523 \mathrm{~K}$ отношение $\zeta$ для $E=10,1$ и 0.1 мэВ и $\gamma=90^{\circ}$ оказалось равным $1.66635,0.93289$ и 0.33547 соответственно.

Чтобы объяснить, в чем состоит ошибка в рассуждении [11], введем и напомним следующие понятия и обозначения. Если $r$ - расстояние между ядрами ионов $\mathrm{K}^{+}$и Г, то «длина» молекулы КІ есть $\ell=r+R_{1}+R_{2}$, и центр масс молекулы становится ближе к поверхности на расстояние $\ell$ за время $t_{\text {transl }}=-\ell / u$, где $u$ дается формулой (17). Вращательная постоянная молекулы, рассматриваемой как жесткий ротатор, есть $B_{r}=\hbar^{2} /\left(2 m_{\mathrm{red}} r^{2}\right)$, a угловая скорость вращения молекулы, отвечающая вращательному квантовому числу $J \geq 0$, есть

$$
\omega_{\mathrm{rot}}(J)=\frac{\hbar[J(J+1)]^{1 / 2}}{m_{\mathrm{red}} r^{2}},
$$

при этом вероятность такого квантового числа пропорциональна

$$
w(J)=(2 J+1) \exp \left(-\frac{B_{r} J(J+1)}{k_{\mathrm{B}} T_{r}}\right) .
$$

Период вращения молекулы, отвечающий вращательному квантовому числу $J$, есть $t_{\text {rot }}(J)=2 \pi / \omega_{\text {rot }}(J)$. При этом $\omega_{\text {rot }}(0)=0$ и $t_{\text {rot }}(0)=\infty$.

Кроме того, расстояние $\Lambda$ от центра масс молекулы до «самой дальней» точки иона равно

$$
\Lambda_{1}=\frac{m_{2} r}{m_{1}+m_{2}}+R_{1} \quad \text { и } \quad \Lambda_{2}=\frac{m_{1} r}{m_{1}+m_{2}}+R_{2}
$$

для ионов $\mathrm{K}^{+}$и I' соответственно (ср. формулу (13)). Конечно, $\Lambda_{1}+\Lambda_{2}=\ell$.

Утверждение [11] “С точки зрения вращений и колебаний молекулы ее ориентация и фаза колебаний в момент первого соударения с поверхностью графита полностью случайны” верно только в том случае, когда, грубо говоря, $t_{\text {rot }}(J)>t_{\text {transl }}$ для типичных $J$. Если это не так, то ион, для которого расстояние $\Lambda$ больше, «получает дополнительное преимущество» в первом соударении с поверхностью, и отношение $\zeta$ «смещается в сторону» этого иона (увеличивается при $\Lambda_{2}>\Lambda_{1}$ и уменьшается при $\Lambda_{1}>\Lambda_{2}$ ).

Чтобы это увидеть, рассмотрим двумерную задачу - жесткий стержень, который движется в полуплоскости $z>0$ плоскости с координатами $(x, z)$, вращаясь вокруг своего центра масс с угловой скоростью $\omega_{\text {rot }} \geq 0$ (и периодом $t_{\text {rot }}=2 \pi / \omega_{\text {rot }}$ ) и приближаясь к оси $z=0$ со скоростью $u_{*}>0$. Занумеруем концы стержня цифрами 1 и 2 и обозначим через 
$\Lambda_{1}$ и $\Lambda_{2}<\Lambda_{1}$ расстояния от центра масс стержня до концов 1 и 2 . Если скорость $u_{*}$ бесконечна или угловая скорость $\omega_{\text {rot }}$ равна нулю, то, очевидно, стержень с равной вероятностью может удариться об ось $z=0$ концом 1 и концом 2. В случае же конечных значений $u_{*}$ и $t_{\text {rot }}$ вероятность удара стержня об ось $z=0$ концом 1 больше - тем больше, чем больше отношение $\left(\Lambda_{1}-\Lambda_{2}\right) / u_{*} t_{\text {rot }}=t_{\text {crit }} / t_{\text {rot }}$, где $t_{\text {crit }}=\left(\Lambda_{1}-\Lambda_{2}\right) / u_{*}$. В момент удара стержня об ось $z=0$ его центр масс находится от оси на расстоянии, не большем $\Lambda_{1}$, а если стержень ударился об эту ось концом 2 , то его центр масс в этот момент находится от оси на расстоянии, не большем $\Lambda_{2}$. Поэтому если за время $t_{\text {crit }}$ стержень успевает сделать полный оборот: $t_{\text {crit }} \geq t_{\text {rot }}$, то стержень заведомо ударится об ось $z=0$ концом 1 , а вероятность удара об ось концом 2 равна нулю. Если центр масс стержня находился от оси на расстоянии $\Lambda_{1}$ в момент времени $t_{\bullet}$, а удар конца 1 стержня об ось произошел в момент времени $t_{0}$, то $0 \leq t_{0}-t_{\bullet} \leq t_{\text {rot }} \leq t_{\text {crit }}$.

Этот пример подсказывает, что условие $t_{\text {rot }}(J)>t_{\text {transl }}=\left(\Lambda_{1}+\Lambda_{2}\right) /|u|$, скореe всего, можно заменить на условие, что $t_{\text {rot }}(J)$ существенно больше, чем $t_{\text {crit }}=\left|\Lambda_{1}-\Lambda_{2}\right| /|u|$.

Среднее значение расстояния $r$ в молекуле КІ для температур $T_{v}=600 \mathrm{~K}$ и $T_{r}=70 \mathrm{~K}$ равно 5.7987 $\left( \pm 10^{-4}\right)$ Бор. Положим $r$ равным этому значению. Тогда

$$
\Lambda_{1}=6.9463>\Lambda_{2}=5.5043 \text { Бор. }
$$

Величина (19), как показывают вычисления, максимальна при $J=20$, и значения $t_{\text {rot }}(1), t_{\text {rot }}(20)$ и $t_{\text {rot }}(100)$ равны $8.140 \times 10^{6}, 5.617 \times 10^{5}$ и $1.145 \times 10^{5}$ a. е. соответственно. Значение же $t_{\text {transl }}$ при $E \sin ^{2} \gamma=10,1,10^{-1}, 10^{-2}, 10^{-3}$ и $10^{-4}$ эВ равно $7.989 \times 10^{3}$, $2.526 \times 10^{4}, 7.989 \times 10^{4}, 2.526 \times 10^{5}, 7.989 \times 10^{5}$ и $2.526 \times 10^{6}$ a. е. соответственно. Поэтому $\zeta \approx 1.7805$ при энергиях $E \sin ^{2} \gamma \geq 0.1$ эВ, а когда $E \sin ^{2} \gamma$ становится меньше $\approx 0.1$ эВ, отношение $\zeta$ начинает «смещаться в сторону» иона $\mathrm{K}^{+}$, т. е. падать.

\section{3. Проблема соответствия экспериментальным данным}

Хотя, как мы отметили во введении, от импульсного приближения обычно в принципе нельзя ожидать хорошего воспроизведения экспериментальных данных, вопрос о том, можно ли полученные в нашей лаборатории данные по диссоциативному рассеянию молекул КI на поверхности графита и алмаза описать с помощью рассматриваемого класса импульсных моделей, безусловно, является важным.

Наши экспериментальные данные по диссоциации KI на поверхности графита носят довольно ограниченный характер $[9,11]$, в частности, в эксперименте угол $\gamma$ падения пучка молекул KI и угол $\varphi_{j}$, под которым измеряется вылет ионов детектором (см. формулу (14)), всегда были дополнительными: $\gamma+\varphi_{j}=90^{\circ}$. Такая геометрия была обусловлена тем, что источник пучка и детектор в эксперименте были фиксированы, а поворачивался держатель, на котором был установлен образец из высокоориентированного пиролитического графита. Недостаточность экспериментальных данных затрудняет сравнение динамических характеристик диссоциации, рассчитанных в рамках импульсной модели раздела 5 , с результатами эксперимента. Тем не менее в работе [11] отмечены существенные расхождения между экспериментальными и расчетными данными. Эти расхождения касаются как зависимости вероятности диссоциации от температуры поверхности $T_{s}$, так и поведения функций $f_{j}(\gamma)$, равных интенсивности вылета $j$-ого иона $(j=1,2)$ на угле $\varphi_{j}=90^{\circ}-\gamma$ для пучка молекул 
KI с углом падения $\gamma$ (конечно, эти функции зависят также от энергии молекул $E$ в пучке и от температуры $\left.T_{s}\right)$. При этом расчетные функции $f_{2}(\gamma)$, относящиеся к иону $\mathrm{I}^{-}$, обнаруживают большее сходство с экспериментальными, чем расчетные функции $f_{1}(\gamma)$, относящиеся к иону $\mathrm{K}^{+}$(предположительно в связи с меньшей массой иона калия). Вопрос о том, можно ли все эти расхождения устранить или хотя бы уменьшить путем другого выбора «столкновительных» $M_{n, j}$ и «максвелловских» $\mu_{n, j}$ масс (отличного от выбора (10)-(12)), остается открытым.

Что же касается наших экспериментальных данных по диссоциации KI на поверхности алмаза (где ограничение $\gamma+\varphi_{j}=$ const отсутствовало), то они однозначно показывают, что в процессе диссоциации существенную роль играют тангенщиальные силы, т. е. действующие на ионы силы, параллельные поверхности. Это очевидно из того, что «горизонтальная» компонента импульса системы ионов $\mathrm{K}^{+}-\mathrm{I}^{-}$в наших экспериментах оказывалась намного меньше, чем «горизонтальная» компонента импульса исходной молекулы КI. Поэтому рассеяние молекул KI на поверхности алмаза невозможно адекватно описать с помощью рассматриваемых импульсных моделей (ни при каком выборе масс $M_{n, j}$ и $\mu_{n, j}$ ), равно как и с помощью любых моделей, в которых учитываются только «нормальные» силы, ортогональные поверхности.

В качестве примера на рис. 3 представлены экспериментальные энергетические распределения ионов $\mathrm{K}^{+}$и $\mathrm{I}^{-}$, образовавшихся при диссоциации молекул $\mathrm{KI}$ на поверхности алмаза при температуре поверхности $T_{s}=250^{\circ} \mathrm{C}$, энергии молекул в пучке $E=12$ эВ, угле падения пучка $\gamma=35^{\circ}$ и углах рассеяния ионов, отвечающих максимальной величине сигнала $\left(\varphi_{1}=45^{\circ}\right.$ для $\mathrm{K}^{+}$и $\varphi_{2}=25^{\circ}$ для $\left.\mathrm{I}^{-}\right)$. При этом энергии ионов почти не менялись при переходе к другим углам падения пучка $\gamma\left(20^{\circ}, 25^{\circ}, 30^{\circ}, 40^{\circ}\right)$ и другим углам рассеяния $\varphi_{j}$, что может показаться удивительным. Однако подобные результаты наблюдались в статье [49] при поверхностно-индуцированной диссоциации ионных комплексов $\mathrm{FeCp}_{2}^{+}$и $\mathrm{SiMe}_{3}^{+}$ (где через $\mathrm{Cp}$ и Ме обозначены циклопентадиен $\mathrm{C}_{5} \mathrm{H}_{6}$ и метильная группа $\mathrm{CH}_{3}$ соответственно) на слоях $\mathrm{C}_{3} \mathrm{H}_{6}, \mathrm{C}_{3} \mathrm{~F}_{6}$ и $\mathrm{C}_{2} \mathrm{Cl}_{4}$. В этой работе было обнаружено, что энергии ионовфрагментов, на которые распадаются исходные ионные комплексы, не зависят от углов падения в $25^{\circ}, 45^{\circ}$ и $65^{\circ}$, а только от природы поверхности - пропилена, гексафторпропилена или тетрахлорэтилена.

В эксперименте мы регистрировали только ионы, отклонение скорости которых от плоскости $O x z$ очень мало. Однако если скорость одного из ионов, образовавшихся при диссоциации молекулы KI, мало отклоняется от плоскости $O x z$, то скорость другого иона в отсутствие тангенциальных сил также мало отклоняется от этой плоскости (в противном случае $y$-компонента импульса системы ионов, равная нулю для исходной молекулы, будет заметно отличаться от нуля после диссоциации, что невозможно без тангенциальных сил). Поэтому при анализе наличия тангенциальных сил, действующих на ионы, мы можем считать, что некоторые молекулы KI, падающие на алмаз с энергией $E=12$ эВ под углом $\gamma=35^{\circ}$ в плоскости $O x z$, диссоциируют с образованием свободных ионов $\mathrm{K}^{+}$и $\mathrm{I}^{-}$, скорости которых мало отклоняются от этой плоскости и энергии которых имеют распределения, близкие к показанным на рис. 3 (напомним, что экспериментальные энергетические распределения ионов были примерно одинаковые на всех углах рассеяния $\varphi_{1}$ и $\varphi_{2}$ ). Таким образом, энергии $E_{1}$ и $E_{2}$ обоих ионов $\mathrm{K}^{+}$и $\mathrm{I}^{-}$в этой ситуации почти всегда не превышают $E_{\max }=3.5$ эВ. Импульс исходной молекулы KI имеет $x$-компоненту, равную $p_{a}=\left[2\left(m_{1}+m_{2}\right) E\right]^{1 / 2} \cos \gamma, \quad$ а конечная $x$-компонента системы ионов равна 
$p_{b}=\left(2 m_{1} E_{1}\right)^{1 / 2} \cos \varphi_{1}+\left(2 m_{2} E_{2}\right)^{1 / 2} \cos \varphi_{2}$ (на самом деле она немного меньше этой величины, так как скорости ионов всё-таки чуть отклоняются от плоскости $O x z$ ). Следовательно,

$$
\frac{p_{b}}{p_{a}}=\frac{\left(2 m_{1} E_{1}\right)^{1 / 2} \cos \varphi_{1}+\left(2 m_{2} E_{2}\right)^{1 / 2} \cos \varphi_{2}}{\left[2\left(m_{1}+m_{2}\right) E\right]^{1 / 2} \cos \gamma}<\frac{\left(2 m_{1} E_{\max }\right)^{1 / 2}+\left(2 m_{2} E_{\max }\right)^{1 / 2}}{\left[2\left(m_{1}+m_{2}\right) E\right]^{1 / 2} \cos \gamma}=0.8964 .
$$

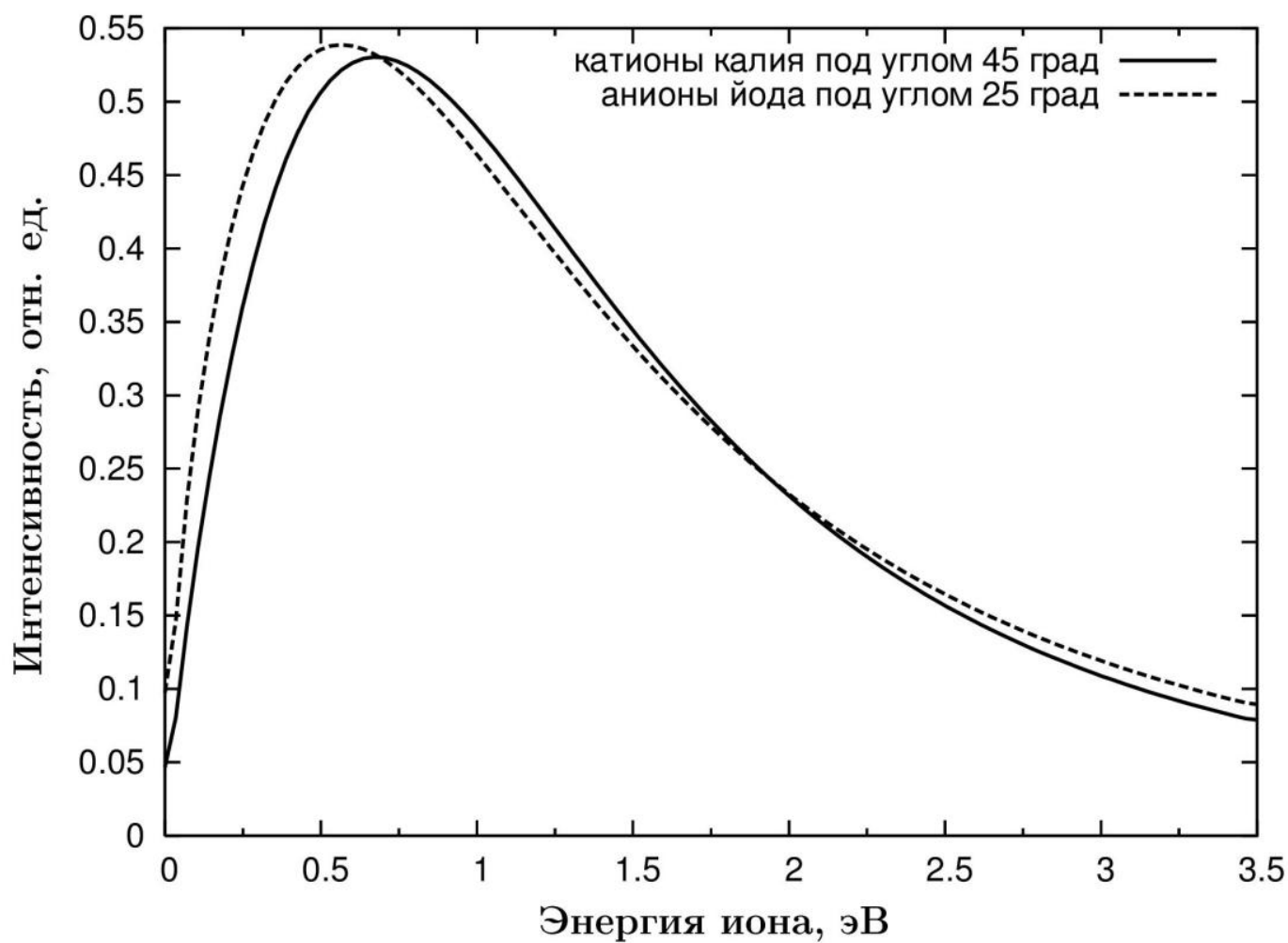

Рис. 3. Экспериментальные энергетические распределения ионов $\mathrm{K}^{+}$и $\mathrm{I}^{-}$, образовавшихся при диссоциации молекул КІ на поверхности алмаза при $E=12$ эВ,

$$
\gamma=35^{\circ}, T_{s}=250^{\circ} \mathrm{C}, \varphi_{1}=45^{\circ}, \varphi_{2}=25^{\circ}
$$

В действительности, конечно, этот эффект уменьшения «горизонтальной» компоненты импульса системы ионов выражен намного сильнее, потому что для подавляющего большинства молекул KI энергии $E_{1}$ и $E_{2}$ существенно меньше $E_{\max }$, а величины $\cos \varphi_{1}$ и $\cos \varphi_{2}$ значительно меньше единицы. Например, если $\varphi_{1}=45^{\circ}, \varphi_{2}=25^{\circ}$ (углы рассеяния ионов, отвечающие максимальной величине сигнала) и $E_{1}=0.67172$ эВ, $E_{2}=0.56566$ эВ (положения максимумов распределений на рис. 3), то $p_{b} / p_{a}=0.3091$. При переходе к меньшим углам $\gamma$ рассматриваемый эффект становится еще более впечатляющим. Итак, на молекулу KI при ее диссоциации на поверхности алмаза (110) действуют заметные силы, параллельные поверхности (и направленные противоположно полету молекулы).

\section{7. Корреляционный анализ постоянных моделей рассеяния молекул КI}

\section{1. Определение постоянных моделей}

В этом разделе мы еще сильнее сузим класс импульсных моделей рассеяния молекул KI на твердой поверхности, а именно, мы будем рассматривать только модели, для которых «столкновительные» $M_{n, j}$ и «максвелловские» $\mu_{n, j}$ массы не зависят от номера $n$ удара 
иона об условную частицу поверхности в рамках процедуры составных соударений (см. подраздел 3.2):

$$
M_{n, 1}=M_{\mathrm{K}^{+}}, \quad M_{n, 2}=M_{\mathrm{I}^{-}}, \quad \mu_{n, 1}=\mu_{\mathrm{K}^{+}}, \quad \mu_{n, 2}=\mu_{\mathrm{I}^{-}} .
$$

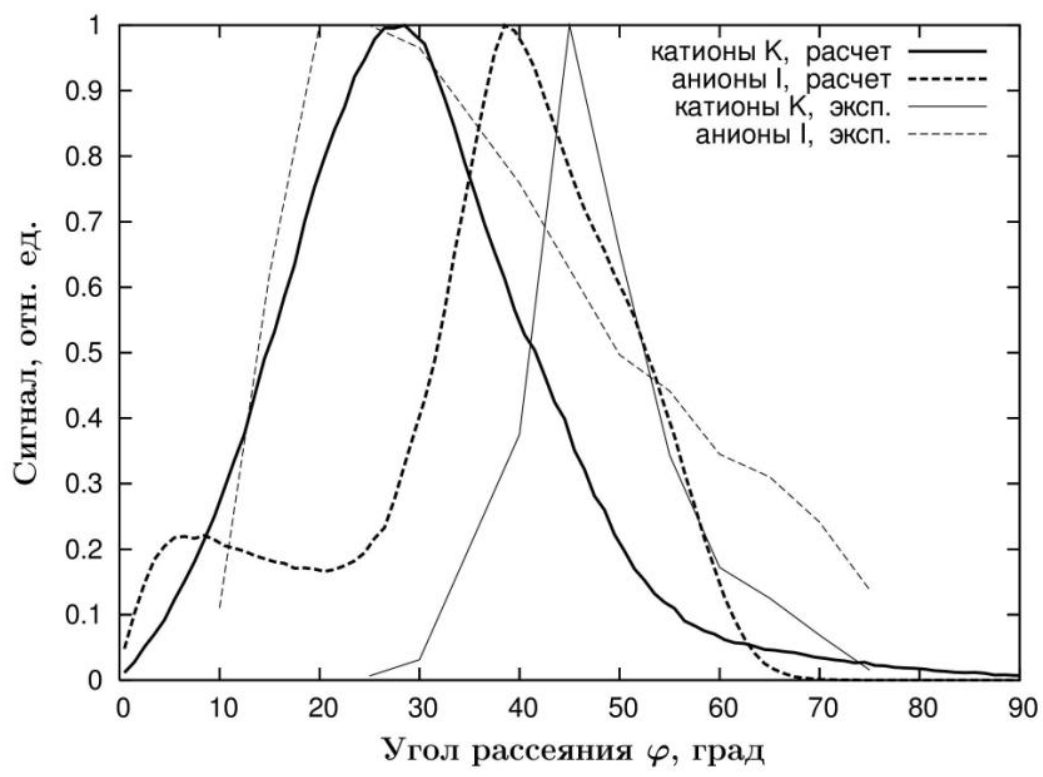

Рис. 4. Угловые распределения ионов $\mathrm{K}^{+}$и $\mathrm{I}^{-}$, для которых $\vartheta_{j} \leq 2^{\circ}$, при $E=12.09$ эВ $\gamma=35^{\circ}, T_{s}=523 \mathrm{~K}$ для «столкновительных» и «максвелловских» масс, заданных равенствами (10), (18), и экспериментальные угловые распределения ионов при диссоциации молекул KI на поверхности алмаза с $E=12$ эB $, \gamma=35^{\circ}, T_{s}=250^{\circ} \mathrm{C}$

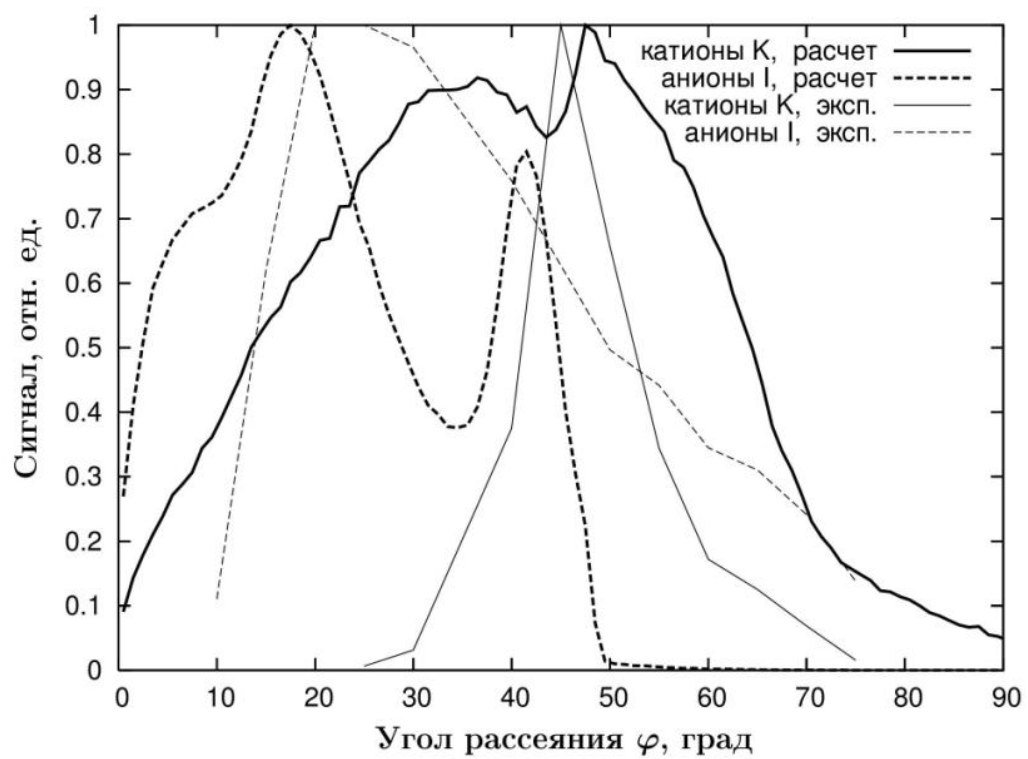

Рис. 5. Угловые распределения ионов $\mathrm{K}^{+}$и $\mathrm{I}^{-}$, для которых $\vartheta_{j} \leq 2^{\circ}$, при $E=12.09$ эВ $, \gamma=35^{\circ}, T_{s}=523 \mathrm{~K}$ для «столкновительных» и «максвелловских» масс $\left(M_{\mathrm{K}^{+}}, M_{\mathrm{I}^{-}}, \mu_{\mathrm{K}^{+}}, \mu_{\mathrm{I}^{-}}\right)=\left(10^{4}, 10^{4}, 4.6,5 \times 10^{4}\right.$ Да) и экспериментальные угловые распределения ионов при диссоциации молекул КI на поверхности алмаза с $E=12$ эВ, $\gamma=35^{\circ}, T_{s}=250^{\circ} \mathrm{C}$ 
Такие модели мы будем называть постоянными. Если $M_{\mathrm{K}^{+}}>0, M_{\mathrm{I}^{-}}>0, \mu_{\mathrm{K}^{+}}<\infty$, $\mu_{\mathrm{I}^{-}}<\infty$, то согласно теоремам подраздела 3.4 всякое соударение ионов с поверхностью включает только конечное число упругих ударов. Модель, определяемая выбором (10), (18) «столкновительных» и «максвелловских» масс, относится к числу постоянных: для нее $M_{\mathrm{K}^{+}}=M_{\mathrm{I}^{-}}=\infty$ и $\mu_{\mathrm{K}^{+}}=\mu_{\mathrm{I}^{-}}=m_{\mathrm{C}}$. Подчеркнем, что, за исключением «столкновительных» и «максвелловских» масс, все постоянные модели ничем не отличаются от модели, изложенной в разделе 5 (это относится и к колебательной $T_{v}=600 \mathrm{~K}$ и вращательной $T_{r}=70 \mathrm{~K}$ температурам молекул).

Даже в рамках класса постоянных моделей динамические характеристики диссоциативного рассеяния молекул КІ на поверхности оказываются очень разнообразными. На рис. 4 и 5 в качестве примера для двух разных наборов масс приведены нормированные на единицу угловые распределения ионов $\mathrm{K}^{+}$и $\Gamma$, для которых $\vartheta_{j} \leq 2^{\circ}$ (см. подраздел 5.5), при энергии молекул в пучке $E=12.09$ эВ, угле падения пучка $\gamma=35^{\circ}$ и температуре поверхности $T_{s}=523 \mathrm{~K}\left(\approx 250^{\circ} \mathrm{C}\right)$. Рис. 4 отвечает выбору $(10)$, (18) «столкновительных» и «максвелловских» масс (с интегрированием $5.62 \times 10^{7}$ траекторий), а рис. 5 - массам $M_{\mathrm{K}^{+}}=M_{\mathrm{I}^{-}}=10^{4}$ Да, $\mu_{\mathrm{K}^{+}}=4.6$ Да и $\mu_{\mathrm{I}^{-}}=5 \times 10^{4}$ Да (было проинтегрировано $10^{8}$ траекторий). Здесь и в подразделе 7.4 ниже «некруглые» значения объема статистики наподобие $5.62 \times 10^{7}$ объясняются тем, что мы в параметрах вычислительной задачи задавали объем равным $10^{8}$, но в соответствии с особенностями выполнения пакетных заданий расчет «обрывался», как только реальное время выполнения задания (“wall time”) достигало 48 часов. Для сравнения на рис. 4 и 5 показаны также экспериментальные угловые распределения ионов $\mathrm{K}^{+}$и Г, образующихся при диссоциации молекул KI на поверхности алмаза при энергии молекул в пучке $E=12$ эВ, угле падения пучка $\gamma=35^{\circ}$ и температуре поверхности $T_{s}=250^{\circ} \mathrm{C}$.

Расчетные угловые распределения ионов на рис. 4 и на рис. 5 имеют мало общего. В частности, на рис. 4 максимумы расчетных угловых распределений «переставлены» по сравнению с максимумами экспериментальных, а на рис. 5 максимумы расчетных и экспериментальных угловых распределений близки. Бимодальность расчетных угловых распределений ионов на рис. 4 и 5, особенно резко выраженная для углового распределения иона $\mathrm{I}^{-}$на рис. 5, весьма типична для самых разных задач химической физики. Мы ограничимся лишь одним примером: молекулярно-пучковыми исследованиями взаимодействия атомов $\mathrm{K}, \mathrm{Rb}$ и $\mathrm{Cs}$ с молекулами тетрахлорметана $\mathrm{CCl}_{4}$ и хлороформа $\mathrm{CHCl}_{3}$ [50] с бимодальными угловыми распределениями образующихся хлоридов $\mathrm{KCl}, \mathrm{RbCl}$ и $\mathrm{CsCl}$ в лабораторной системе координат.

Отметим, что в случае выбора (10)-(12) «столкновительных» и «максвелловских» масс при $E=12$ эВ, $\gamma=35^{\circ}$ и $T_{s}=523 \mathrm{~K}$ мы наблюдали лишь единичные «диссоциативные» траектории ( $N_{d}=21$ на $10^{7}$ всех траекторий).

\section{2. Общая схема расчета коэффициентов корреляций}

Одним из формальных математических приемов, облегчающих изучение связей между различными величинами, описывающими то или иное явление, является подсчет коэффициентов корреляций [7, 51-54]. Предположим, что мы исследуем некоторый процесс, который в рамках данного подхода определяется конечным набором параметров $\zeta_{(1)}, \zeta_{(2)}, \ldots, \zeta_{(L)}$. Например, речь может идти о параметрах поверхности потенциальной энергии, управляющей какой-либо (гомогенной или гетерогенной) элементарной химиче- 
ской реакцией (или неупругим взаимодействием) в химической физике [7, 55-57], или же (что представляет основной интерес в контексте настоящей статьи) о «столкновительных» $M_{\mathrm{K}^{+}}, M_{\mathrm{I}^{-}}$и «максвелловских» $\mu_{\mathrm{K}^{+}}, \mu_{\mathrm{I}^{-}}$массах в постоянных импульсных моделях рассеяния молекул KI на твердой поверхности. Нас интересует некоторая динамическая характеристика $\sigma$ рассматриваемого процесса, которая является функцией параметров $\zeta_{(1)}, \zeta_{(2)}, \ldots, \zeta_{(L)}$ :

$$
\sigma=f\left(\zeta_{(1)}, \zeta_{(2)}, \ldots, \zeta_{(L)}\right)
$$

Зависимость $f$ может быть очень сложной (и малообозримой при $L>1$ ), но корреляционный метод позволяет определить основные тенденции в поведении функции $f$ «с высоты птичьего полета» (в линейном приближении). Для этого в простейшем случае надо построить (с помощью той или иной процедуры Монте-Карло) «достаточно представительное» семейство наборов параметров

$$
\left(\zeta_{(1), \alpha}, \zeta_{(2), \alpha}, \ldots, \zeta_{(L), \alpha}\right), \quad 1 \leq \alpha \leq K,
$$

для каждого из этих $K$ наборов найти соответствующее значение $\sigma_{\alpha}$ величины $\sigma$ и рассчитать эмпирические коэффищиенты коррелящий

$$
r\left(\sigma, \zeta_{(i)}\right)=\frac{\left\langle\sigma \zeta_{(i)}\right\rangle-\langle\sigma\rangle\left\langle\zeta_{(i)}\right\rangle}{\left[\left\langle\sigma^{2}\right\rangle-\langle\sigma\rangle^{2}\right]^{1 / 2}\left[\left\langle\zeta_{(i)}^{2}\right\rangle-\left\langle\zeta_{(i)}\right\rangle^{2}\right]^{1 / 2}}, \quad 1 \leq i \leq L .
$$

Угловыми скобками в формуле (21) обозначены средние по семейству из $K$ наборов параметров:

$$
\begin{gathered}
\left\langle\zeta_{(i)}\right\rangle=\frac{1}{K} \sum_{\alpha=1}^{K} \zeta_{(i), \alpha}, \quad\left\langle\zeta_{(i)}^{2}\right\rangle=\frac{1}{K} \sum_{\alpha=1}^{K} \zeta_{(i), \alpha}^{2}, \\
\langle\sigma\rangle=\frac{1}{K} \sum_{\alpha=1}^{K} \sigma_{\alpha}, \quad\left\langle\sigma \zeta_{(i)}\right\rangle=\frac{1}{K} \sum_{\alpha=1}^{K} \sigma_{\alpha} \zeta_{(i), \alpha}, \\
\left\langle\sigma^{2}\right\rangle=\frac{1}{K} \sum_{\alpha=1}^{K} \sigma_{\alpha}^{2} .
\end{gathered}
$$

Коэффициент корреляции (21) заключен между -1 и 1 и отражает точность наилучшей аппроксимации величины $\sigma$ линейной функцией $a \zeta_{(i)}+b$, а также знак множителя $a$ в такой аппроксимации. Близость коэффициента корреляции $r\left(\sigma, \zeta_{(i)}\right)$ к единице означает наличие выраженной тенденции увеличения $\sigma$ с ростом параметра $\zeta_{(i)}$, а близость этого коэффициента корреляции к -1 означает наличие выраженной обратной тенденции уменьшения $\sigma \mathrm{c}$ ростом параметра $\zeta_{(i)}$.

Если параметры $\zeta_{(1)}, \zeta_{(2)}, \ldots, \zeta_{(L)}$ независимы, то по коэффициентам корреляций (21) легко определить эмпирический множественный (употребляются также термины сводный, полный или совокупный) коэффициент корреляции между б и набором $\zeta_{(1)}, \zeta_{(2)}, \ldots, \zeta_{(L)}$ :

$$
R\left(\sigma ; \zeta_{(1)}, \zeta_{(2)}, \ldots, \zeta_{(L)}\right)=\left(\sum_{i=1}^{L}\left[r\left(\sigma, \zeta_{(i)}\right)\right]^{2}\right)^{1 / 2} .
$$


Коэффициент корреляции (24) заключен между 0 и 1 и отражает точность наилучшей аппроксимации величины $\sigma$ линейной функцией $\sum_{i=1}^{L} a_{i} \zeta_{(i)}+b$.

\section{3. Эффективные алгоритмы расчета коэффициентов корреляций}

Рассмотрим теперь более подробно ситуацию, когда для определения величины $\sigma$, соответствующей данному набору значений параметров $\zeta_{(1)}, \zeta_{(2)}, \ldots, \zeta_{(L)}$, не существует никакой детерминированной процедуры, но эта величина равна математическому ожиданию М некоторой случайной величины $\Phi$. О единичном акте розыгрыша этой величины мы будем говорить как об интегрировании «траектории», имея в виду, что во многих задачах химической физики речь идет о настоящих траекториях, каждой из которых сопоставляется определенное значение величины $\Phi$.

Например, пусть $\sigma$ - сечение некоторого бимолекулярного процесса, управляемого данной поверхностью потенциальной энергии и моделируемого методом квазиклассических траекторий, причем прицельный параметр траектории разыгрывается по правилу $b=b_{\max } \chi^{\lambda}$, где $b_{\max }$ - максимальное значение прицельного параметра, $\chi-$ случайная величина, равномерно распределенная между 0 и 1 , а $\lambda>0-$ фиксированная константа. Тогда при «наиболее естественной» процедуре розыгрыша всех остальных начальных условий траектории имеет место равенство $\sigma=\mathbf{M} \Phi$, где $\Phi=2 \pi \lambda\left(b_{\max } b^{2 \lambda-1}\right)^{1 / \lambda}$, если данная траектория отвечает изучаемому процессу, и $\Phi=0$ в противном случае $[7,55]$. Обычно используются показатели $\lambda=1 / 2$ (когда $\Phi$ принимает одно и то же значение $\pi b_{\max }^{2}$ для всех траекторий, отвечающих изучаемому процессу) и $\lambda=1$. Если $\sigma-$ вероятность $P$ диссоциации молекулы KI для данной постоянной импульсной модели, то $\sigma=\mathbf{M} \Phi$, где $\Phi=1$ для «диссоциативных» траекторий и $\Phi=0$ для «недиссоциативных». Если $\sigma-$ вероятность того, что молекула KI диссоциировала, причем угол $\vartheta_{j}$ отклонения конечной скорости $j$-ого иона $(j=1,2)$ от плоскости $O x z$ не превосходит $2^{\circ}$ (см. подраздел 5.5), то $\sigma=\mathbf{M \Phi , ~ г д е ~}$ $\Phi=1$ для «диссоциативных» траекторий, удовлетворяющих условию $\vartheta_{j} \leq 2^{\circ}$, и $\Phi=0$ для остальных траекторий.

Если для каждого набора (20) значений параметров интегрируется «достаточно большое» число $N$ траекторий, то в формулах (22) и (23) логично положить

$$
\sigma_{\alpha}=\frac{1}{N} \sum_{\beta=1}^{N} \Phi_{\alpha, \beta}, \quad 1 \leq \alpha \leq K,
$$

где $\Phi_{\alpha, \beta}-$ значение величины $\Phi$ для $\beta$-ой траектории, интегрируемой с набором параметров $\left(\zeta_{(1), \alpha}, \zeta_{(2), \alpha}, \ldots, \zeta_{(L), \alpha}\right)$. В частности,

$$
\begin{gathered}
\sigma_{\alpha} \zeta_{(i), \alpha}=\frac{1}{N} \sum_{\beta=1}^{N} \Phi_{\alpha, \beta} \zeta_{(i), \alpha}, \quad 1 \leq \alpha \leq K, \\
\sigma_{\alpha}^{2}=\left(\frac{1}{N} \sum_{\beta=1}^{N} \Phi_{\alpha, \beta}\right)^{2}, \quad 1 \leq \alpha \leq K .
\end{gathered}
$$

При этом каждый коэффициент корреляции (21) превращается в коэффициент корреляции между двумя последовательностями из $K$ чисел: $\zeta_{(i), \alpha}, 1 \leq \alpha \leq K$ и (25).

В диссертации [7] (см. §§ 3.1г), 3.4а) и 5.6 этой диссертации), в статьях [55, 57] и в отчете [56] мы рассмотрели вопрос о том, можно ли при вычислении коэффициентов корре- 
ляций (21) и (24) ограничиться интегрированием небольшого числа $N$ траекторий для каждого набора (20) значений параметров, «скомпенсировав» малость $N$ очень большим числом $K$ самих наборов. В качестве величины $\sigma$ мы брали полное сечение обменной реакции $\mathrm{F}+\mathrm{H}_{2}(v=J=0) \rightarrow \mathrm{H}+\mathrm{HF}[7,55]$ и сечения различных каналов реакции $\mathrm{CsCl}+\mathrm{RbI}[7,55-$ 57], а в качестве параметров $\zeta_{(1)}, \zeta_{(2)}, \ldots, \zeta_{(L)}$ - параметры соответствующих поверхностей потенциальной энергии. При этом в работах [55] (см. в той статье стр. 9), [56] (см. в том отчете стр. 16) и [57] (см. в той статье стр. 8) мы утверждали, что при подстановке в формулы (22) и (23) значений (25)-(27) будут получаться правильные значения средних $\langle\sigma\rangle,\left\langle\sigma \zeta_{(i)}\right\rangle$ и $\left\langle\sigma^{2}\right\rangle$ даже при малых $N$, если число $K$ разыгранных наборов параметров достаточно велико.

Это утверждение неверно (и не подтверждается результатами расчетов, приведенных в [55-57]). Подставляя (25) и (26) в (22) при малом $N$, но очень большом $K$, мы действительно имеем основания получить адекватные значения $\langle\sigma\rangle$ и $\left\langle\sigma \zeta_{(i)}\right\rangle$, но при подстановке (27) в (23) это уже не так.

В самом деле, пусть $\mathbf{M}_{\alpha}$ - математическое ожидание по траекториям, интегрируемым с набором параметров $\left(\zeta_{(1), \alpha}, \zeta_{(2), \alpha}, \ldots, \zeta_{(L), \alpha}\right)$, так что $\sigma_{\alpha}=\mathbf{M}_{\alpha} \Phi$ и $\sigma_{\alpha}=\mathbf{M}_{\alpha} \Phi_{\alpha, \beta}$ для каждого индекса $\beta(1 \leq \beta \leq N)$. Тогда, конечно,

$$
\mathbf{M}_{\alpha}\left(\frac{1}{N} \sum_{\beta=1}^{N} \Phi_{\alpha, \beta}\right)=\sigma_{\alpha}, \quad \mathbf{M}_{\alpha}\left(\frac{1}{N} \sum_{\beta=1}^{N} \Phi_{\alpha, \beta} \zeta_{(i), \alpha}\right)=\sigma_{\alpha} \zeta_{(i), \alpha} .
$$

Однако равенство

$$
\mathbf{M}_{\alpha}\left[\left(\frac{1}{N} \sum_{\beta=1}^{N} \Phi_{\alpha, \beta}\right)^{2}\right]=\sigma_{\alpha}^{2}
$$

уже не имеет места. Действительно, считая все величины $\Phi_{\alpha, 1}, \Phi_{\alpha, 2}, \ldots, \Phi_{\alpha, N}$ независимыми, мы находим

$$
\begin{aligned}
& \mathbf{M}_{\alpha}\left[\left(\frac{1}{N} \sum_{\beta=1}^{N} \Phi_{\alpha, \beta}\right)^{2}\right]-\left[\mathbf{M}_{\alpha}\left(\frac{1}{N} \sum_{\beta=1}^{N} \Phi_{\alpha, \beta}\right)\right]^{2}=\mathbf{D}_{\alpha}\left(\frac{1}{N} \sum_{\beta=1}^{N} \Phi_{\alpha, \beta}\right), \\
& \mathbf{D}_{\alpha}\left(\frac{1}{N} \sum_{\beta=1}^{N} \Phi_{\alpha, \beta}\right)=\frac{1}{N^{2}} \mathbf{D}_{\alpha}\left(\sum_{\beta=1}^{N} \Phi_{\alpha, \beta}\right)=\frac{1}{N^{2}} \sum_{\beta=1}^{N} \mathbf{D}_{\alpha} \Phi_{\alpha, \beta}=\frac{\mathbf{D}_{\alpha} \Phi}{N},
\end{aligned}
$$

где $\mathbf{D}_{\alpha}=\mathbf{M}_{\alpha} \circ \mathrm{Sq}-\mathrm{Sq} \circ \mathbf{M}_{\alpha}$ - дисперсия (Sq - оператор возведения в квадрат). С учетом (28) получаем

$$
\mathbf{M}_{\alpha}\left[\left(\frac{1}{N} \sum_{\beta=1}^{N} \Phi_{\alpha, \beta}\right)^{2}\right]=\sigma_{\alpha}^{2}+\frac{\mathbf{D}_{\alpha} \Phi}{N}
$$

откуда при больших $K$

$$
\frac{1}{K} \sum_{\alpha=1}^{K}\left(\frac{1}{N} \sum_{\beta=1}^{N} \Phi_{\alpha, \beta}\right)^{2} \approx\left\langle\sigma^{2}\right\rangle+\frac{\langle\mathbf{D} \Phi\rangle}{N}
$$


где

$$
\langle\mathbf{D} \Phi\rangle=\frac{1}{K} \sum_{\alpha=1}^{K} \mathbf{D}_{\alpha} \Phi
$$

При малых $N$ дополнительное слагаемое $\langle\mathbf{D} \Phi\rangle / N>0$ в (31) невозможно «скомпенсировать» никаким увеличением $K$. Это слагаемое приведет к систематическому занижению коэффициентов корреляций.

Например, пусть для какого-то $\alpha$ величина $\Phi$ принимает только два значения: $1 \mathrm{c}$ вероятностью $p$ и 0 с вероятностью $1-p$, так что $\sigma_{\alpha}=\mathbf{M}_{\alpha} \Phi=p$. Пусть, кроме того, $N=1$. В этом простейшем случае несостоятельность равенства (29) очевидна: так как $\Phi^{2}=\Phi$, то $\mathbf{M}_{\alpha}\left(\Phi^{2}\right)$ равно не $\sigma_{\alpha}^{2}=p^{2}$, а $p$. Разность $p-p^{2}$ есть в точности $\mathbf{D}_{\alpha} \Phi$ в полном соответствии с (30).

Если при всех $\alpha$ величина $\Phi$ принимает всего два значения: 1 с вероятностью $p_{\alpha}$ и 0 с вероятностью $1-p_{\alpha}$, то $\sigma_{\alpha}=\mathbf{M}_{\alpha} \Phi=p_{\alpha}$ и $\mathbf{D}_{\alpha} \Phi=\mathbf{M}_{\alpha}\left(\Phi^{2}\right)-\left(\mathbf{M}_{\alpha} \Phi\right)^{2}=p_{\alpha}-p_{\alpha}^{2}=\sigma_{\alpha}-\sigma_{\alpha}^{2}$ при всех $\alpha$, так что формула (31) с учетом (25) превращается в

$$
\frac{1}{K} \sum_{\alpha=1}^{K}\left(\frac{1}{N} \sum_{\beta=1}^{N} \Phi_{\alpha, \beta}\right)^{2} \approx \frac{N-1}{N}\left\langle\sigma^{2}\right\rangle+\frac{1}{N}\langle\sigma\rangle \approx \frac{N-1}{N}\left\langle\sigma^{2}\right\rangle+\frac{1}{N K} \sum_{\alpha=1}^{K}\left(\frac{1}{N} \sum_{\beta=1}^{N} \Phi_{\alpha, \beta}\right) .
$$

Конечно, приближенные равенства (32) имеют место лишь при больших $K$.

В § 3.1г) диссертации [7] мы предложили другой способ оценки $\left\langle\sigma^{2}\right\rangle$, заключающийся в том, что число $N$ предполагается четным, а в формуле (23) вместо (27) используется приближение

$$
\sigma_{\alpha}^{2}=\frac{2}{N} \sum_{\beta=1}^{N / 2} \Phi_{\alpha, 2 \beta-1} \Phi_{\alpha, 2 \beta}, \quad 1 \leq \alpha \leq K
$$

При подстановке (33) в (23) мы получим правильное значение среднего $\left\langle\sigma^{2}\right\rangle$ даже при малых четных $N$, если число $K$ разыгранных наборов параметров достаточно велико (это подтверждается результатами расчетов, приведенных в [7, 55-57]). Действительно, снова считая все величины $\Phi_{\alpha, 1}, \Phi_{\alpha, 2}, \ldots, \Phi_{\alpha, N}$ независимыми, мы находим

$$
\mathbf{M}_{\alpha}\left(\frac{2}{N} \sum_{\beta=1}^{N / 2} \Phi_{\alpha, 2 \beta-1} \Phi_{\alpha, 2 \beta}\right)=\frac{2}{N} \sum_{\beta=1}^{N / 2} \mathbf{M}_{\alpha}\left(\Phi_{\alpha, 2 \beta-1} \Phi_{\alpha, 2 \beta}\right)=\frac{2}{N} \sum_{\beta=1}^{N / 2}\left(\mathbf{M}_{\alpha} \Phi_{\alpha, 2 \beta-1}\right)\left(\mathbf{M}_{\alpha} \Phi_{\alpha, 2 \beta}\right)=\sigma_{\alpha}^{2},
$$

откуда при больших $K$

$$
\frac{1}{K} \sum_{\alpha=1}^{K}\left(\frac{2}{N} \sum_{\beta=1}^{N / 2} \Phi_{\alpha, 2 \beta-1} \Phi_{\alpha, 2 \beta}\right) \approx\left\langle\sigma^{2}\right\rangle \text {. }
$$

Недостатком расчета $\sigma_{\alpha}^{2}$ по формуле (33) является то, что получающиеся при этом величины $r\left(\sigma, \zeta_{(i)}\right)$ уже не являются, вообще говоря, коэффициентами корреляций никаких фактических двумерных распределений. Поэтому возможны ситуации, когда $\left\langle\sigma^{2}\right\rangle \leq\langle\sigma\rangle^{2}$, так что коэффициенты (21) вообще не определены, или когда $\left.\left\langle\sigma^{2}\right\rangle\right\rangle\langle\sigma\rangle^{2}$, но коэффициенты (21) оказываются по модулю больше единицы для некоторых $i$ (или же все коэффициен- 
ты (21) по модулю меньше единицы, но множественный коэффициент (24) больше единицы). Соответствующие примеры приведены в § 3.1г) диссертации [7] (см. также раздел 5 отчета [56]), еще один пример нам встретится ниже в следующем подразделе. Если коэффициент (21) для какого-то $i$ получается по модулю больше единицы, то, как правило, это означает, что соответствующий «настоящий» коэффициент корреляции по модулю близок к 1 , а его знак совпадает со знаком коэффициента (21).

Если при всех $\alpha$ величина $\Phi$ принимает всего два значения: 1 и 0 , то, подставляя (34) в (32), мы после умножения на $K N^{2}$ получаем (при больших $K$ ) соотношение

$$
\sum_{\alpha=1}^{K}\left(\sum_{\beta=1}^{N} \Phi_{\alpha, \beta}\right)^{2} \approx 2(N-1) \sum_{\alpha=1}^{K} \sum_{\beta=1}^{N / 2} \Phi_{\alpha, 2 \beta-1} \Phi_{\alpha, 2 \beta}+\sum_{\alpha=1}^{K} \sum_{\beta=1}^{N} \Phi_{\alpha, \beta} .
$$

При $N=2$ это равенство является точным для любого $K$, потому что для любого $\alpha$ выполнено тождество $\left(\Phi_{\alpha, 1}+\Phi_{\alpha, 2}\right)^{2}=2 \Phi_{\alpha, 1} \Phi_{\alpha, 2}+\Phi_{\alpha, 1}+\Phi_{\alpha, 2}$. Другими словами, если при всех $\alpha$ величина $\Phi$ принимает только два значения: 1 и 0 , а $N=2$, то при любом $K$ результат подстановки (27) в формулу (23) для $\left\langle\sigma^{2}\right\rangle$ - это в точности среднее арифметическое результата подстановки (33) в формулу (23) для $\left\langle\sigma^{2}\right\rangle$ и результата подстановки (25) в формулу (22) для $\langle\sigma\rangle$.

\section{4. Приложения к постоянным моделям}

В настоящей статье мы ограничимся тем, что проиллюстрируем материал подразделов 7.2 и 7.3 на двух примерах, относящихся к постоянным импульсным моделям рассеяния молекул KI на твердой поверхности. В качестве параметров $\zeta_{(i)}(1 \leq i \leq L=4)$ в этих примерах выступают «столкновительные» $M_{\mathrm{K}^{+}}, M_{\mathrm{I}^{-}}$и «максвелловские» $\mu_{\mathrm{K}^{+}}, \mu_{\mathrm{I}^{-}}$массы, которые равномерно разыгрываются в пределах

$$
\begin{gathered}
10^{2} \leq M_{\mathrm{K}^{+}} \leq 5 \times 10^{4} \text { Да, } 10^{2} \leq M_{\mathrm{I}^{-}} \leq 5 \times 10^{4} \text { Да, } \\
4 \leq \mu_{\mathrm{K}^{+}} \leq 10^{2} \text { Да, } 4 \leq \mu_{\mathrm{I}^{-}} \leq 10^{2} \text { Да. }
\end{gathered}
$$

Рассматриваются три динамические характеристики рассеяния $\sigma=\mathbf{M \Phi ~ : ~}$

a) $\sigma$ - вероятность $P$ диссоциации молекулы KI, при этом $\Phi=1$ для «диссоциативных» траекторий и $\Phi=0$ для «недиссоциативных».

б) $\sigma$ - вероятность того, что молекула KI диссоциировала, причем угол $\vartheta_{1}$ отклонения конечной скорости иона $\mathrm{K}^{+}$от плоскости $O x z$ не превосходит $2^{\circ}$ (см. подраздел 5.5); тогда $\Phi=1$ для «диссоциативных» траекторий, удовлетворяющих условию $\vartheta_{1} \leq 2^{\circ}$, и $\Phi=0$ для остальных траекторий.

в) $\sigma$ - вероятность того, что молекула КІ диссоциировала, причем угол $\vartheta_{2}$ отклонения конечной скорости иона I' от плоскости $O x z$ не превосходит $2^{\circ}$; тогда $\Phi=1$ для «диссоциативных» траекторий, удовлетворяющих условию $\vartheta_{2} \leq 2^{\circ}$, и $\Phi=0$ для остальных траекторий.

В первом примере мы при энергии молекул в пучке $E=12.09$ эВ, угле падения пучка $\gamma=35^{\circ}$ и температуре поверхности $T_{s}=523 \mathrm{~K}$ рассчитали суммы (31) и (34) для шести значений числа $N$ траекторий, разыгрываемых для каждого набора масс

$$
\left(\zeta_{(1)}, \zeta_{(2)}, \zeta_{(3)}, \zeta_{(4)}\right)=\left(M_{\mathrm{K}^{+}}, M_{\mathrm{I}^{-}}, \mu_{\mathrm{K}^{+}}, \mu_{\mathrm{I}^{-}}\right) \text {, }
$$


а именно, для $N=2,4,10,20,200$ и 2000. Количество $K$ самих наборов при этом было равно $31598100,14284200,6804800,4225100,443200$ и 47600 соответственно. Результаты вычислений представлены на рис. 6. Как и следовало ожидать, сумма (34) для каждой из трех характеристик $\sigma$ практически не зависит от $N$, в то время как сумма (31) монотонно убывает с ростом $N$, асимптотически приближаясь к (34) при $N \rightarrow \infty$, и зависит от $1 / N$ практически линейно.

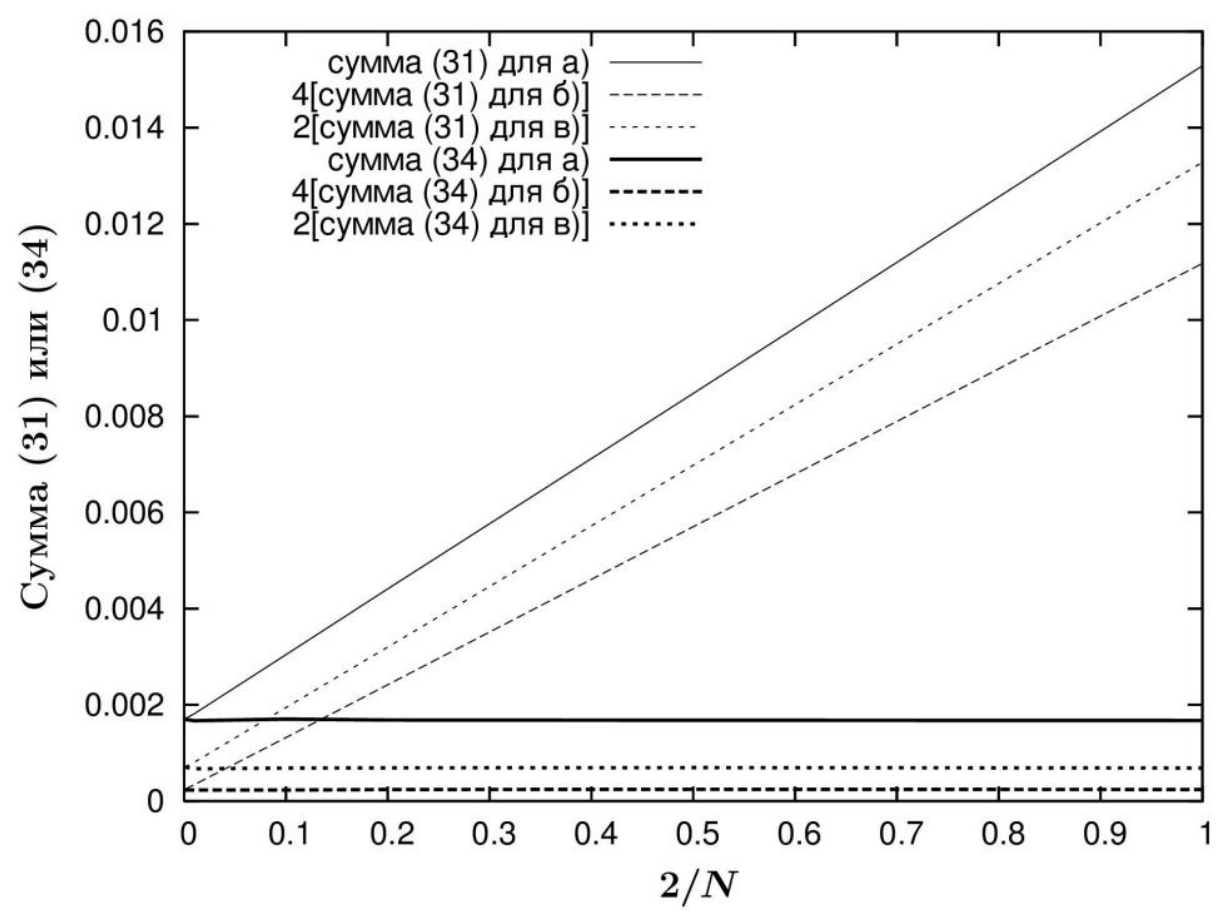

Рис. 6. Зависимость сумм (31) и (34) от числа $N$ траекторий, разыгрываемых для каждого набора масс, при $E=12.09$ эВ, $\gamma=35^{\circ}, T_{s}=523 \mathrm{~K}$ для трех характеристик а)-в). Запись “4[сумма (31) для б)]” означает, что данная кривая показывает умноженные на 4 значения суммы (31) для характеристики б); обозначения для других кривых аналогичны

Во втором примере мы при энергиях молекул в пучке $E$ от 0.5 до 15 эВ с шагом 0.5 эВ, угле падения пучка $\gamma=90^{\circ}$ и температуре поверхности $T_{s}=523 \mathrm{~K}$ рассчитали коэффициенты корреляций (21) и множественный коэффициент корреляции (24), разыгрывая для каждого набора масс всего две траектории $(N=2)$ и вычисляя средние (22) и (23) в соответствии с формулами (25), (26) и (33):

$$
\begin{gathered}
\langle\sigma\rangle=\frac{1}{2 K} \sum_{\alpha=1}^{K}\left(\Phi_{\alpha, 1}+\Phi_{\alpha, 2}\right), \quad\left\langle\sigma \zeta_{(i)}\right\rangle=\frac{1}{2 K} \sum_{\alpha=1}^{K}\left(\Phi_{\alpha, 1}+\Phi_{\alpha, 2}\right) \zeta_{(i), \alpha}, \\
\left\langle\sigma^{2}\right\rangle=\frac{1}{K} \sum_{\alpha=1}^{K} \Phi_{\alpha, 1} \Phi_{\alpha, 2} .
\end{gathered}
$$

Количество $K$ самих наборов масс при этом было разным при разных энергиях $E$ и менялось в пределах от $2.4 \times 10^{6}$ до $4.12 \times 10^{7}$ (и в среднем было чуть меньше $3.1 \times 10^{7}$ ).

Результаты вычислений представлены на рис. 7-9. Для характеристики б) при $E=8.5$ эВ имело место неравенство

$$
9.980315 \times 10^{-5}=\left\langle\sigma^{2}\right\rangle\left\langle\langle\sigma\rangle^{2}=\left(1.0035748 \times 10^{-2}\right)^{2}=1.0071624 \times 10^{-4} .\right.
$$


В этом случае мы условно считали, что множественный коэффициент корреляции (24) равен 1 , а коэффициенты корреляций (21) равны 1 или -1 в зависимости от знака числителя в (21) (см. рис. 8). При $E=14.5$ эВ для характеристики б) выполнялось «правильное» неравенство $\left\langle\sigma^{2}\right\rangle>\langle\sigma\rangle^{2}$ и все четыре коэффициента корреляций (21) по модулю были меньше единицы, но множественный коэффициент корреляции $R=1.030109$ оказался больше единицы, и мы считали, что $R=1$ (см. рис. 8).

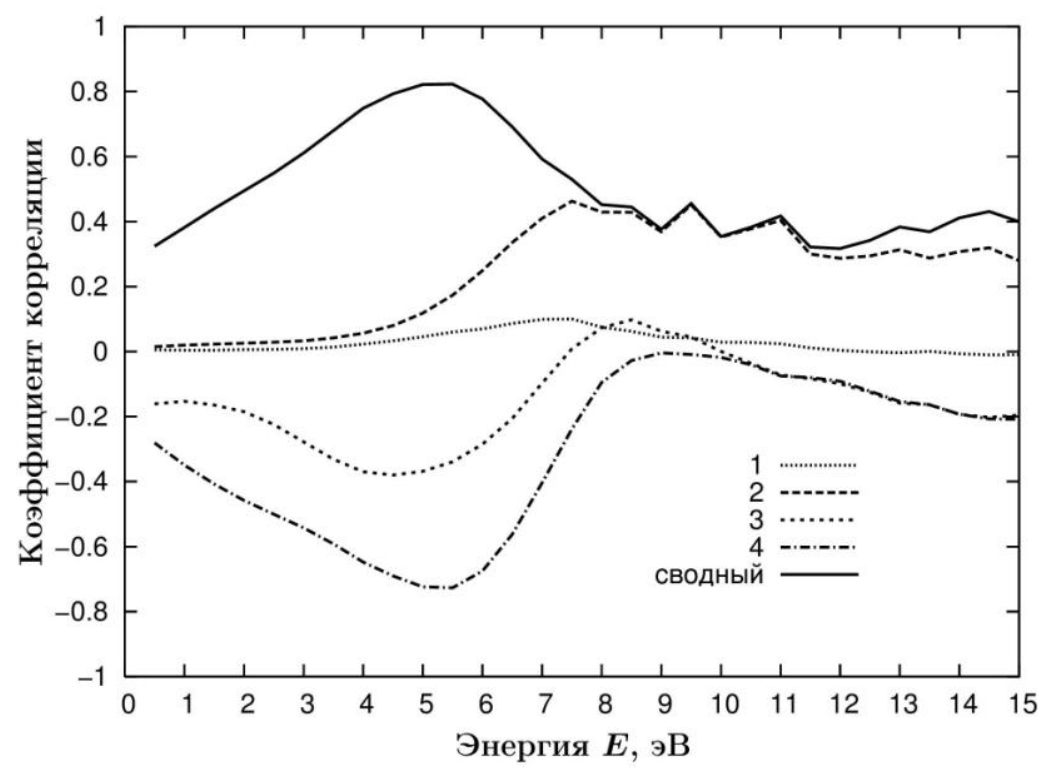

Рис. 7. Коэффициенты корреляций между вероятностью диссоциации $P$ и массами $M_{\mathrm{K}^{+}}$(кривая 1$), M_{\mathrm{I}^{-}}$(кривая 2), $\mu_{\mathrm{K}^{+}}$(кривая 3), $\mu_{\mathrm{I}^{-}}$(кривая 4), а также сводный (множественный) коэффициент корреляции при разных значениях энергии $E$ и при $\gamma=90^{\circ}, T_{s}=523 \mathrm{~K}$

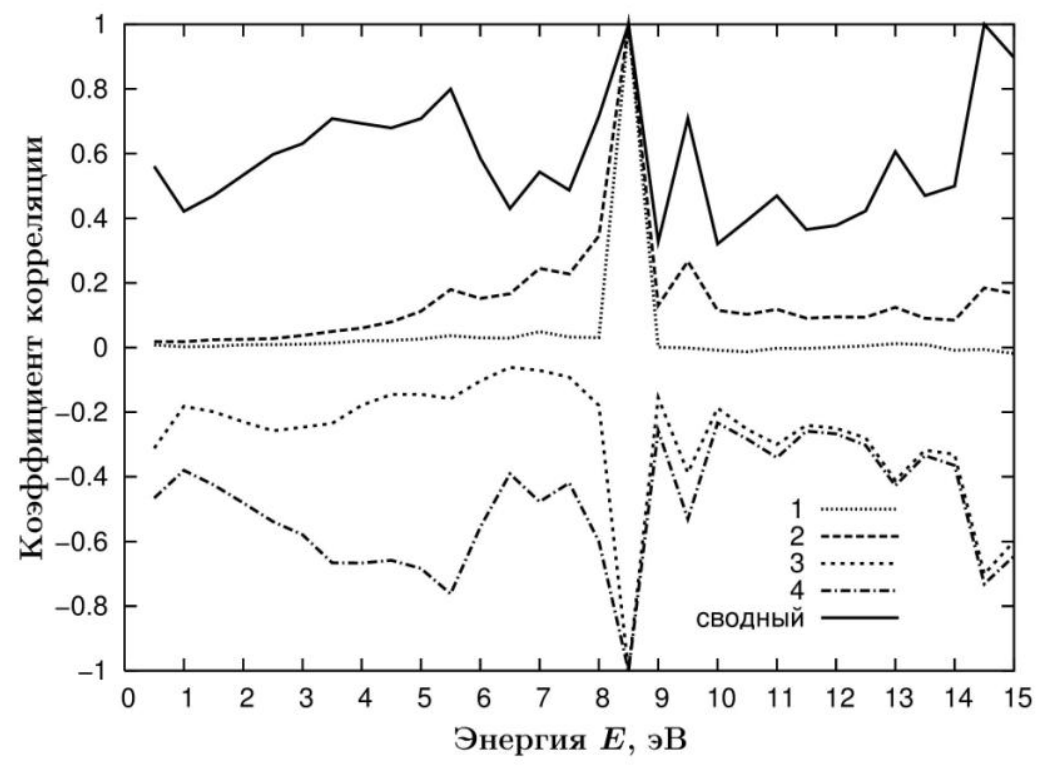

Рис. 8. Коэффициенты корреляций между вероятностью диссоциации с $\vartheta_{1} \leq 2^{\circ}$ и массами $M_{\mathrm{K}^{+}}$(кривая 1$), M_{\mathrm{I}^{-}}$(кривая 2$), \mu_{\mathrm{K}^{+}}$(кривая 3$), \mu_{\mathrm{I}^{-}}$ (кривая 4), а также сводный (множественный) коэффициент корреляции при разных значениях энергии $E$ и при $\gamma=90^{\circ}, T_{s}=523 \mathrm{~K}$ 
Из рис. 7 видно, что (во всяком случае при указанных пределах изменения масс $M_{\mathrm{K}^{+}}$, $M_{\mathrm{I}^{-}}, \mu_{\mathrm{K}^{+}}, \mu_{\mathrm{I}^{-}}$, при $0.5 \leq E \sin ^{2} \gamma \leq 15$ эВ и при $T_{s}=523 \mathrm{~K}$ ) вероятность $P$ диссоциации молекулы КІ в целом увеличивается с ростом $M_{\mathrm{I}^{-}}$и уменьшается с ростом $\mu_{\mathrm{K}^{+}}$и $\mu_{\mathrm{I}^{-}}$, причем зависимость $P$ от $\mu_{\mathrm{I}^{-}}$сильнее, чем от $\mu_{\mathrm{K}^{+}}$. Ограничения $\vartheta_{j} \leq 2^{\circ}(j=1,2)$ при $\gamma=90^{\circ}$ оказывают мало влияния на эти тенденции (рис. 8 и 9).

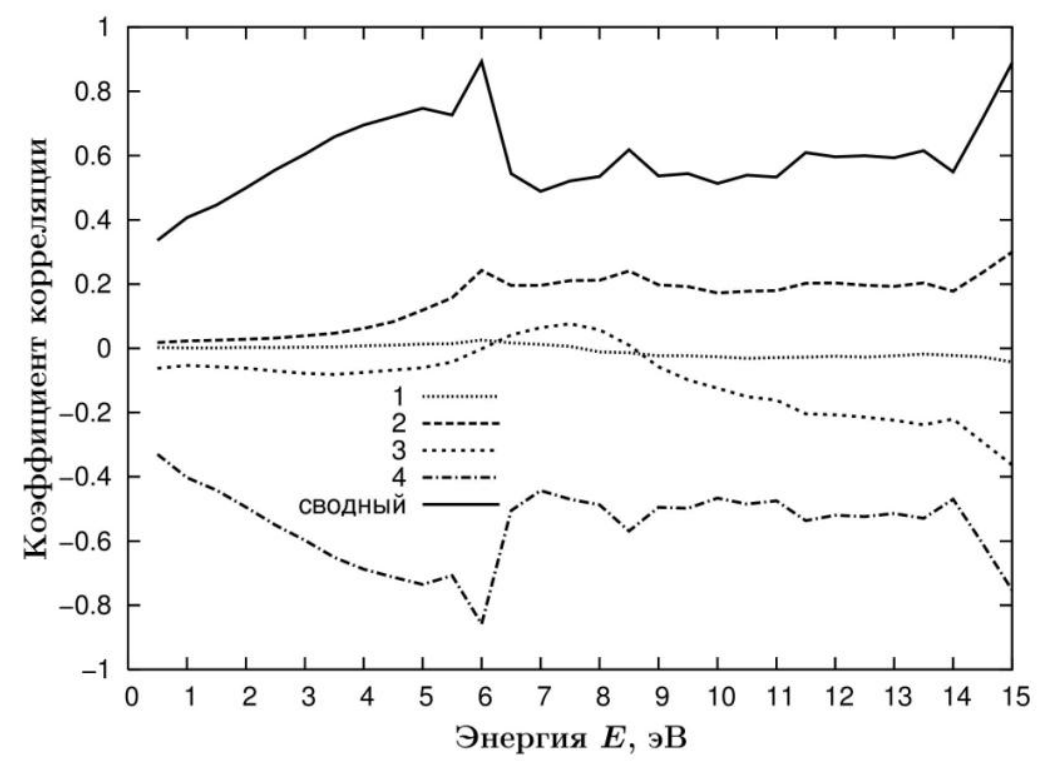

Рис. 9. Коэффициенты корреляций между вероятностью диссоциации с $\vartheta_{2} \leq 2^{\circ}$ и массами $M_{\mathrm{K}^{+}}$(кривая 1$), M_{\mathrm{I}^{-}}$(кривая 2), $\mu_{\mathrm{K}^{+}}$(кривая 3$), \mu_{\mathrm{I}^{-}}$ (кривая 4), а также сводный (множественный) коэффициент корреляции при разных значениях энергии $E$ и при $\gamma=90^{\circ}, T_{s}=523 \mathrm{~K}$

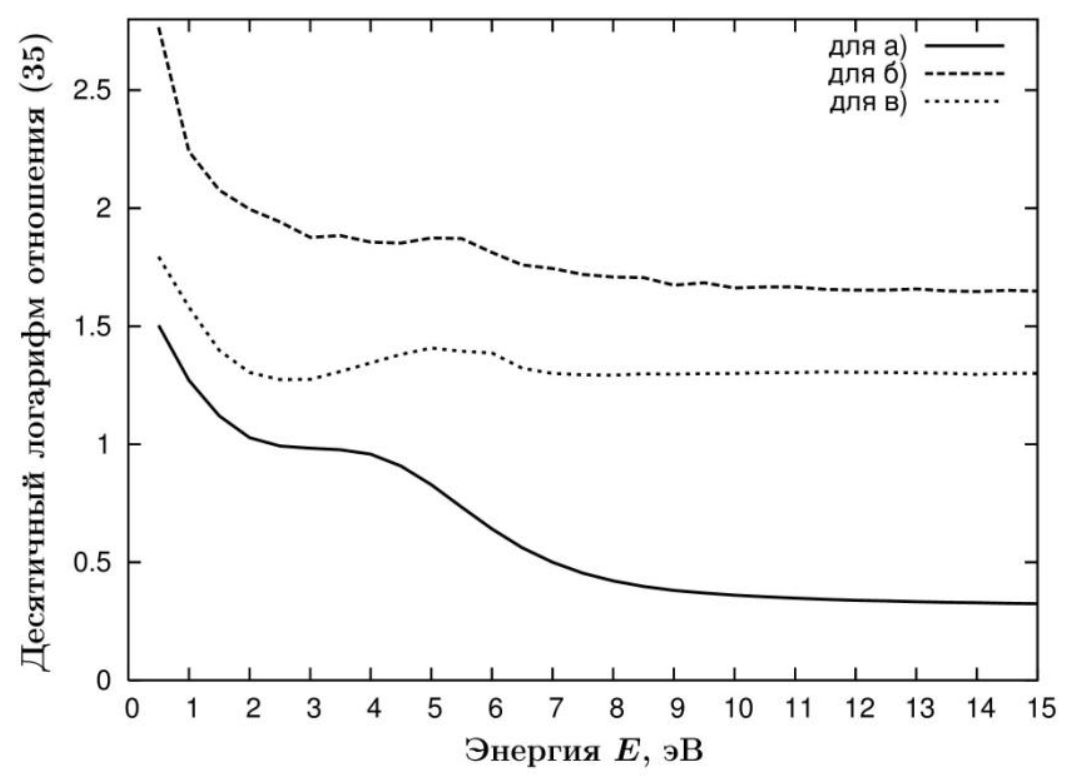

Рис. 10. Отношение (35) для трех характеристик а)-в) при разных значениях энергии $E$ и при $\gamma=90^{\circ}, T_{s}=523 \mathrm{~K}$ 
Для каждой из трех характеристик $\sigma$ средние $\langle\sigma\rangle$ и $\left\langle\sigma^{2}\right\rangle$ увеличиваются с ростом энергии $E-$ во всем исследованном диапазоне значений $E$ для характеристики а), при $E \leq 9$ эВ для характеристики б) и при $E \leq 8$ эВ для характеристики в). Для каждого фиксированного значения энергии $E$ (вплоть до 15 эВ) значение $\langle\sigma\rangle$ для характеристики а), конечно, больше, чем для характеристики в), а для характеристики в) больше, чем для характеристики б) - по-видимому, за счет большей массы иона йода. Та же закономерность справедлива для значений $\left\langle\sigma^{2}\right\rangle$.

На рис. 10 для всех трех характеристик а)-в) представлена зависимость от энергии $E$ отношения сумм (31) и (34), т. е. величины

$$
\sum_{\alpha=1}^{K}\left(\Phi_{\alpha, 1}+\Phi_{\alpha, 2}\right)^{2} /\left(4 \sum_{\alpha=1}^{K} \Phi_{\alpha, 1} \Phi_{\alpha, 2}\right)>1 .
$$

Отметим, что согласно (32) с учетом сказанного в конце предыдущего подраздела это отношение при любом $K$ равно

$$
\frac{1}{2}+\frac{\langle\sigma\rangle}{2\left\langle\sigma^{2}\right\rangle}
$$

Как видно из рис. 10, для каждой из трех характеристик отношение (35) в целом уменьшается с ростом энергии $E$. Для каждого фиксированного значения энергии $E$ отношение (35) для характеристики а) меньше, чем для характеристики в), а для характеристики в) меньше, чем для характеристики б). Причина этого состоит в том, что отношение (35) в целом тем больше, чем меньше вероятность $\sigma\left(\langle\sigma\rangle /\left\langle\sigma^{2}\right\rangle\right.$ имеет порядок $\left.1 / \sigma\right)$.

\section{8. Интегральное описание рассеяния и условие взаимности}

В настоящей статье мы совершенно не касались чрезвычайно важного вопроса о том, какое распределение может иметь «вертикальная» компонента $u_{\perp}$ скорости атома, получающаяся в результате процедуры составных соударений $C E\left(m,\left\{M_{n}\right\},\left\{\mu_{n}\right\}, T_{s}, u_{0}\right)$ (в тех случаях, когда вероятность бесконечной серии ударов равна нулю, см. подраздел 3.4$)$. Этот весьма нетривиальный с математической точки зрения вопрос является темой дальнейших исследований и публикаций. Отметим, тем не менее, что любая (не обязательно импульсная) модель рассеяния атомов или ионов на твердой поверхности однозначно задается соответствием между распределением скоростей атомов, падающих на поверхность, и атомов, отраженных от поверхности (хотя механизм взаимодействия атома с поверхностью при таком описании часто остается в тени).

Предположим, что поверхность «макроскопически» представляет собой плоскость $z=0$ и имеет некоторую температуру $T_{s}$, а атомы массы $m$ движутся в полупространстве $z>0$. Скорость $\tilde{\mathbf{u}}$ атомов до взаимодействия с поверхностью характеризуется функцией распределения $\tilde{f}(\tilde{\mathbf{u}})$, а скорость и атомов, отраженных от поверхности, - функцией распределения $f(\mathbf{u})$. Конечно, чтобы атом претерпел соударение с поверхностью, $z$-компонента $u_{0}$ его скорости должна быть отрицательной, а $z$-компонента $u_{\perp}$ скорости рассеянного атома положительна. При интегральном описании рассеяния [58-61] соответствие между распределениями $\tilde{f}(\tilde{\mathbf{u}})$ и $f(\mathbf{u})$ выражается формулой

$$
\left|u_{\perp}\right| f(\mathbf{u})=\int_{u_{0}<0}\left|u_{0}\right| \tilde{f}(\tilde{\mathbf{u}}) R(\tilde{\mathbf{u}}, \mathbf{u}) \mathrm{d} \tilde{\mathbf{u}},
$$


где $R(\widetilde{\mathbf{u}}, \mathbf{u})$ - т. н. ядро рассеяния (scattering kernel), зависящее от температуры $T_{s}$, массы $m$ и параметров модели (а также, возможно, от точки соударения атома с поверхностью, если нужно подчеркнуть неоднородность последней). Ядро рассеяния должно удовлетворять условию неотрицательности:

$$
R(\widetilde{\mathbf{u}}, \mathbf{u}) \geq 0
$$

при всех $\tilde{\mathbf{u}}, \mathbf{u}$ с $u_{0}<0$ и $u_{\perp}>0$, а также (если поверхность неадсорбирующая) условию нормировки:

$$
\int_{u_{\perp}>0} R(\tilde{\mathbf{u}}, \mathbf{u}) \mathrm{d} \mathbf{u}=1
$$

при всех $\tilde{\mathbf{u}} \mathbf{c} u_{0}<0$. Интегральное описание часто называют аналитическим, что не надо смешивать с аналитическими выражениями для тех или иных зависимостей, характерными для импульсных моделей (см. введение).

При динамическом задании закона рассеяния ядро рассеяния можно определить численно путем розыгрыша достаточно большого числа актов рассеяния. Примером могут служить расчеты статьи [61], относящиеся к «модели стиральной доски» [21].

Во многих случаях при интегральном описании рассеяния атомов на поверхности на ядро рассеяния $R$ накладывается также условие взаимности (reciprocity), или детального равновесия (detailed balance):

$$
\left|u_{0}\right| F_{3}(\widetilde{\mathbf{u}}) R(\tilde{\mathbf{u}}, \mathbf{u}) \equiv\left|u_{\perp}\right| F_{3}(\mathbf{u}) R(-\mathbf{u},-\widetilde{\mathbf{u}}),
$$

где $F_{3}$ - трехмерное максвелловское распределение, отвечающее температуре $T_{s}$ и массе $m$ :

$$
F_{3}(\mathbf{u})=\left(\frac{m}{2 \pi k_{\mathrm{B}} T_{s}}\right)^{3 / 2} \exp \left(-\frac{m \mathbf{u}^{2}}{2 k_{\mathrm{B}} T_{s}}\right) .
$$

Из условия взаимности (36) (и условия нормировки) вытекает, что если скорости атомов, падающих на поверхность, имеют максвелловское распределение (37), отвечающее температуре поверхности $T_{s}$, то скорости рассеянных атомов имеют то же самое максвелловское распределение (37). Обратное неверно: сохранение максвелловского распределения возможно и без условия взаимности, см. [58] (р. 228), [59] (р. 50) или [60] (р. 114).

В теории рассеяния атомных частиц на поверхности широко используются такие явно заданные ядра рассеяния, удовлетворяющие условию взаимности (36), как ядро Максвелла [62], ядро Эпштейна [63] и ядро Черчиньяни-Лэмпис [64]. Эти ядра и их комбинации и обобщения (тоже удовлетворяющие условию взаимности) обсуждаются, например, в недавних статьях [61, 65-70] (см. также библиографию к этим статьям). В то же время следует подчеркнуть, что в очень многих известных в литературе моделях рассеяния атомов на поверхности условие взаимности нарушается, см., например, [58] (р. 235), [59] (p. 46) или [61] (p. 106), что означает наличие необратимых эффектов при рассеянии.

Удовлетворяют ли условию взаимности импульсные модели взаимодействия атомов с поверхностью, упомянутые в разделе 2, и предложенная в настоящей работе «модель составных соударений»? Насколько известно авторам, вопрос о выполнении условия взаимности в импульсных моделях рассеяния атомов на поверхности (и о том, какой вид имеют соответствующие ядра рассеяния) в литературе систематически не рассматривался. Необходимо отметить, что многие импульсные модели, о которых шла речь в разделе 2, «определены не полностью», а потому для них нарушается даже условие нормировки. Например, в исходной «модели жестких кубов» Логана-Стикни [12] с ненулевой вероятностью атом не 
«отлетает» от поверхности в силу неравенства $u_{\text {new }} \leq 0$ (см. подраздел 2.1). В статье [61] показано, что «модель стиральной доски» [21] условию взаимности не удовлетворяет (но условие нормировки для нее соблюдается). В заметке [71] обсуждаются другие модели рассеяния типа «модели жестких кубов», для которых нарушено условие взаимности.

При некоторых специальных значениях «столкновительных» и «максвелловских» масс $M_{n}$ и $\mu_{n}$ условие взаимности для «модели составных соударений» выполнено. Например, пусть $M_{1}=\mu_{1}=\infty$. Тогда (в обозначениях раздела 3) $U_{1}=0$ и $u_{\perp}=u_{1}=-u_{0}$ (независимо от значений $M_{n}$ и $\mu_{n}$ при $n \geq 2$ ), т. е. рассеяние атома на поверхности представляет собой зеркальное отражение. Соответствующее ядро рассеяния имеет вид $\delta\left(\tilde{\mathbf{u}}-\mathbf{u}+2 u_{\perp} \mathbf{e}_{z}\right)$ (предельный случай ядра Максвелла) и, очевидно, удовлетворяет условию взаимности (здесь $\mathbf{e}_{z}=(0,0,1)$, а $\delta$ - дельта-функция).

Однако в общем случае условие взаимности для «модели составных соударений» (в тех ситуациях, когда серия ударов атома об условные частицы поверхности с вероятностью 1 конечна, что эквивалентно условию нормировки) не выполняется, потому что эта модель, вообще говоря, не сохраняет максвелловское распределение падающих атомов с температурой $T_{s}$. Более того, условие взаимности в общем случае нарушено даже в классе постоянных моделей, для которых массы $M_{n}=M$ и $\mu_{n}=\mu$ не зависят от номера $n$ удара атома об условную частицу поверхности (ср. подраздел 7.1). Причина этого в конечном счете состоит в том свойстве «модели составных соударений» (упомянутом в подразделе 3.2), что, совершенно не меняя динамику взаимодействия атома с поверхностью, мы можем «придать» температуре поверхности любое значение за счет одновременного умножения всех «максвелловских» масс $\mu_{1}, \mu_{2}, \mu_{3}, \ldots$ на одно и то же число.

Более формально, справедлива следующая теорема. Для любой температуры $T_{s}$ и массы $m$, любых констант $M_{\#}>0, \eta<0, \kappa>0$ и любого числа $p$ из интервала $0<p<1$ существует такая константа $\mu_{\#}>0$, что если $\eta \leq u_{0}<0, M_{1} \geq M_{\#}$ и $0<\mu_{1} \leq \mu_{\#}$, то с вероятностью не менее $p$ скорость $u_{1}$, определяемая в рамках процедуры $L S_{2}\left(m, M_{1}, \mu_{1}, T_{s}, u_{0}\right)$, удовлетворяет неравенству $u_{1} \geq \kappa$ (и, таким образом, $\left.u_{\perp}=u_{1} \geq \kappa\right)$.

Действительно, неравенство

$$
u_{1}=\frac{\left(m-M_{1}\right) u_{0}+2 M_{1} U_{1}}{m+M_{1}} \geq \kappa
$$

эквивалентно тому, что

$$
U_{1} \geq \frac{\left(m+M_{1}\right) \kappa+\left(M_{1}-m\right) u_{0}}{2 M_{1}}=\frac{\kappa+u_{0}}{2}+\frac{m\left(\kappa-u_{0}\right)}{2 M_{1}}
$$

(при $M_{1}=\infty$ слагаемое $m\left(\kappa-u_{0}\right) /\left(2 M_{1}\right)$ отсутствует). Если $M_{1} \geq M_{\#}$ и $u_{0} \geq \eta$, то правая часть неравенства (38) не превышает

$$
\Gamma=\max \left(\frac{\kappa}{2}+\frac{m(\kappa-\eta)}{2 M_{\#}},-\eta\right) \geq-\eta \geq\left|u_{0}\right| .
$$

Определим $\mu_{\#}$ с помощью соотношения

$$
\operatorname{erf}\left(\left[\frac{\mu_{\#}}{2 k_{\mathrm{B}} T_{s}}\right]^{1 / 2} \Gamma\right)=1-p
$$


Тогда согласно (5) при $\mu_{1} \leq \mu_{\#}$ вероятность того, что $\left|U_{1}\right| \geq \Gamma$, а значит, и $U_{1} \geq \Gamma$ (и, следовательно, выполнено неравенство (38)), не меньше $p$.

Предположим теперь, что скорости атомов, падающих на поверхность, имеют максвелловское распределение (37), отвечающее температуре поверхности $T_{s}$. Выберем произвольное $\kappa>0$. Согласно только что доказанной теореме при фиксированном значении $M_{1}>0$ можно добиться того, что вероятность неравенства $u_{\perp} \geq \kappa$ (напомним, что $u_{\perp}$ - это $z$-компонента скорости рассеянных атомов) будет сколь угодно близка к 1 (не меньше любого наперед заданного $p<1$ ), положив массу $\mu_{1}$ достаточно малой. Точнее, если задано произвольное число $p$ из интервала $0<p<1$, то надо сначала определить такое $\eta<0$, что неравенство $u_{0} \geq \eta$ для максвелловского распределения с температурой $T_{s}$ выполнено с ве-

роятностью $p^{1 / 2}$, а затем найти такое $\mu_{\#}>0$, что неравенство $u_{\perp} \geq \kappa$ при $u_{0} \geq \eta$ и $\mu_{1} \leq \mu_{\#}$ выполнено с вероятностью не менее $p^{1 / 2}$.

При слишком близкой к 1 вероятности неравенства $u_{\perp} \geq \kappa$ распределение скоростей рассеянных атомов заведомо не совпадает с максвелловским распределением (37). Но если условие взаимности при данных $T_{s}, m$ и $M_{1}>0$ не выполняется при всех достаточно малых $\mu_{1}$ (в тех ситуациях, когда вероятность бесконечной серии ударов равна нулю), то оно не выполняется вообще при всех $\mu_{1}$, кроме, может быть, отдельных исключительных значений (зависящих от масс $M_{n}$ и $\mu_{n}$ с $n \geq 2$ ). В классе постоянных моделей при фиксированном значении $M>0$ условие взаимности нарушается при всех достаточно малых $\mu$, а значит, и при всех $\mu$ вообще, кроме, может быть, отдельных исключительных значений.

Конечно, условие сохранения максвелловского распределения скоростей частиц несущественно при моделировании взаимодействия газодинамического пучка с поверхностью, в особенности если это взаимодействие сопровождается диссоциацией молекул пучка.

\section{9. Заключение}

В заключение перечислим основные результаты работы.

1) Предложена общая импульсная модель рассеяния системы атомных частиц (нейтральных атомов или ионов) на плоской твердой поверхности без тангенциальных сил (т. е. сил, параллельных поверхности). В рамках этой модели предполагается, что каждое отдельное соударение атома с поверхностью представляет собой серию мгновенно следующих друг за другом упругих ударов атома об условные частицы поверхности, которые движутся нормально к последней. Серия характеризуется двумя бесконечными последовательностями масс условных частиц, массой атома, температурой поверхности и нормальной к поверхности компонентой скорости атома непосредственно перед соударением. Сформулированы критерии как конечности, так и бесконечности серий упругих ударов. Модель является обобщением нашей модели рассеяния двухатомных молекул с ионной связью на поверхности графита $[10,11]$ (которая, в свою очередь, основана на «модели жестких кубов» [12]).

2) Установлено, исходя из экспериментальных данных по диссоциации молекул иодида калия на поверхности алмаза (110), что в этом процессе тангенциальные силы играют значительную роль. Таким образом, диссоциативное рассеяние молекул KI на поверхности алмаза невозможно адекватно описать с помощью предложенной модели, но остается открытым вопрос о применимости модели к рассеянию KI на поверхности графита.

Отметим, что одним из путей моделирования тангенциальных сил может быть рассмотрение неровных поверхностей (ср. подраздел 2.2), которые описываются уравнением 
$z=\zeta(x, y)$ с $\langle\zeta\rangle=0$. Даже если силы, действующие со стороны поверхности на атом или ион, нормальны к поверхности в точке соударения $(x, y, \zeta(x, y))$, т. е. коллинеарны вектору $\left(-\partial_{x} \zeta,-\partial_{y} \zeta, 1\right)$, эти силы имеют ненулевую составляющую, параллельную плоскости $z=0$.

3) Проиллюстрировано (на простейших примерах, связанных с рассеянием молекул $\mathrm{KI})$ применение корреляционного анализа к изучению зависимости динамических характеристик рассеяния от масс условных частиц поверхности.

4) Исправлены некоторые неточности предыдущих работ нашей лаборатории, относящиеся, в частности, к вопросу о том, какой из двух ионов $\mathrm{K}^{+}$и $\mathrm{I}^{-}$чаще (и насколько чаще) ударяет о поверхность первым [11], и к возможности вычисления коэффициентов корреляций путем интегрирования малого числа траекторий для каждого набора значений параметров [55-57].

\section{Благодарности}

Авторы благодарны Д.Б. Кабанову за полезные обсуждения и рецензенту за ценные критические замечания. Работа выполнена в рамках Программы фундаментальных научных исследований государственных академий наук на 2013-2020 годы, тема «Фундаментальные физико-химические процессы воздействия энергетических объектов на окружающую среду и живые системы».

\section{Литература}

1. Pérez-Ríos J., Ragole S., Wang J., Greene C.H. Comparison of classical and quantal calculations of helium three-body recombination // J. Chem. Phys. 2014. V. 140. № 4. Paper 044307 (12 p.).

2. Ермолова Е.В., Русин Л.Ю., Севрюк М.Б. Импульсная модель прямой трехтельной рекомбинации тяжелых ионов // Хим. физика. 2014. Т. 33. № 11. С. 12-25.

3. Ермолова Е.В., Русин Л.Ю., Севрюк М.Б. Модель жестких сфер в теории прямой трехтельной рекомбинации ионов. В издании: На стыке наук. Физико-химическая серия. III Международная научная Интернет-конференция (29 января 2015 года). Материалы конференции в двух томах. Сервис виртуальных конференций Рах Grid. Казань: ИП Синяев Д.Н., 2015. Том I. C. $111-121$.

4. Русин Л.Ю., Севрюк М.Б. Простая импульсная модель прямой трехтельной рекомбинации и функция эффективности третьего тела // Физико-химическая кинетика в газовой динамике. 2016. Т. 17, вып. 3. http://chemphys.edu.ru/issues/2016-17-3/articles/667/ (22 c.).

5. Маергойз А.И., Никитин Е.Е., Русин Л.Ю. Динамика образования ионов при столкновительной диссоциации двухатомных молекул. В книге: Химия плазмы. Вып. 12. Под ред. Б.М. Смирнова. М.: Энергоатомиздат, 1985. С. 3-55.

6. Ленин Л.В., Русин Л.Ю., Севрюк М.Б. Процессы диссоциации и комплексообразования при столкновениях молекул галогенидов цезия с атомами инертных газов и ртути: импульсная модель. Деп. в ВИНИТИ 10.12.1991, № 4561-В91. 189 с.

7. Севрюк М.Б. Динамический анализ атомно-молекулярных столкновений. Дисс. на соискание ученой степени докт. физ.-мат. наук. М.: ИНЭПХФ РАН, 2003. 312 с.

8. Русин Л.Ю., Севрюк М.Б. Импульсная модель в теории атомно-молекулярных столкновений: аннотированная библиография вплоть до 1991 года. Отчет в ЦИТиС. Регистрационный номер 215100170008. М.: ИНЭПХФ РАН им. В.Л. Тальрозе, 2015. 108 с.

9. Азриель В.М., Акимов В.М., Колесникова Л.И., Русин Л.Ю., Севрюк М.Б., Хмельницкий Р.А. Диссоциативная ионизация молекул КI на поверхности графита // Наука, техника и образование. 2018. № 3 (44). С. 13-18. 
10. Азриель В.М., Акимов В.М., Колесникова Л.И., Русин Л.Ю., Севрюк М.Б. Импульсная модель диссоциации двухатомных молекул с ионной связью на поверхности графита // Проблемы современной науки и образования. 2018. № 4 (124). С. 48-56.

11. Азриель В.М., Акимов В.М., Ермолова Е.В., Колесникова Л.И., Русин Л.Ю., Севрюк М.Б. Диссоциация иодида калия на поверхности графита в рамках импульсной модели // Хим. физика. 2018. Т. 37. № 11. С. 75-87.

12. Logan R.M., Stickney R.E. Simple classical model for the scattering of gas atoms from a solid surface // J. Chem. Phys. 1966. V. 44. № 1. P. 195-201.

13. Stickney R.E. Atomic and molecular scattering from solid surfaces. In: Advances in Atomic and Molecular Physics. Vol. 3. Eds. D.R. Bates, I. Estermann. N. Y.: Academic Press, 1967. P. 143-204.

14. Goodman F.O. On the theory of accommodation coefficients-IV. Simple distribution function theory of gas-solid interaction systems // J. Phys. Chem. Solids. 1965. V. 26. № 1. P. 85-105.

15. Mateljevic N., Kerwin J., Roy S., Schmidt J.R., Tully J.C. Accommodation of gases at rough surfaces // J. Phys. Chem. C. 2009. V. 113. № 6. P. 2360-2367.

16. Madix R.J., Korus R.A. Trapping effects in gas-solid scattering // J. Phys. Chem. Solids. 1968. V. 29. № 9. P. 1531-1538.

17. Grimmelmann E.K., Tully J.C., Cardillo M.J. Hard-cube model analysis of gas-surface energy accommodation // J. Chem. Phys. 1980. V. 72. № 2. P. 1039-1043.

18. Logan R.M., Keck J.C. Classical theory for the interaction of gas atoms with solid surfaces // J. Chem. Phys. 1968. V. 49. № 2. P. 860-876.

19. Steinbrüchel C. The hard-spheroid model for gas-surface interactions // Chem. Phys. Lett. 1980. V. 76. № 1. P. 58-61.

20. Steinbrüchel C. Gas-surface scattering distributions according to the hard-spheroid model // Surface Science. 1982. V. 115. № 2. P. 247-258.

21. Tully J.C. Washboard model of gas-surface scattering // J. Chem. Phys. 1990. V. 92. № 1. P. 680686.

22. Kummel A.C., Sitz G.O., Zare R.N., Tully J.C. Direct inelastic scattering of $\mathrm{N}_{2}$ from $\mathrm{Ag}(111)$. III. Normal incident $\mathrm{N}_{2}$ // J. Chem. Phys. 1988. V. 89. № 11. P. 6947-6955.

23. Xia L.-Q., Engstrom J.R. The role of surface corrugation in direct translationally activated dissociative adsorption // J. Chem. Phys. 1994. V. 101. № 6. P. 5329-5342.

24. Yan T., Hase W.L., Tully J.C. A washboard with moment of inertia model of gas-surface scattering // J. Chem. Phys. 2004. V. 120. № 2. P. 1031-1043.

25. Doll J.D. Simple classical model for the scattering of diatomic molecules from a solid surface // J. Chem. Phys. 1973. V. 59. № 3. P. 1038-1042.

26. Sitz G.O., Kummel A.C., Zare R.N., Tully J.C. Direct inelastic scattering of $\mathrm{N}_{2}$ from $\operatorname{Ag}(111)$. II. Orientation // J. Chem. Phys. 1988. V. 89. № 4. P. 2572- 2582.

27. Nichols W.L., Weare J.H. Homonuclear diatomic scattering from solid surfaces: A hard-cube model // J. Chem. Phys. 1975. V. 62. № 9. P. 3754-3762.

28. Nichols W.L., Weare J.H. Rotational energy distributions for homonuclear diatomic beams scattered from solid surfaces: A hard-cube model // J. Chem. Phys. 1977. V. 66. № 3. P. 1075-1078.

29. Nichols W.L., Weare J.H. Heteronuclear diatomic scattering from solid surfaces: A hard-cube model // J. Chem. Phys. 1975. V. 63. № 1. P. 379-383.

30. Ionov S.I., Bernstein R.B. Hard-cube analysis of the steric effect in molecule-surface scattering // J. Chem. Phys. 1991. V. 94. № 2. P. 1564-1571.

31. McClure J.D. Atomic and molecular scattering from solids. II. Comparison of classical scattering models in relation to experiment // J. Chem. Phys. 1969. V. 51. № 5. P. 1687-1700.

32. Goodman F.O. Review of the theory of the scattering of gas atoms by solid surfaces // Surface Science. 1971. V. 26. № 1. P. 327-362. 
33. Somorjai G.A., Brumbach S.B. The interaction of molecular beams with solid surfaces // CRC Critical Reviews in Solid State Sciences. 1973. V. 4. № 1-4. P. 429-454.

34. Goodman F.O. Thermal accommodation // Progr. Surf. Sci. 1974. V. 5. № 3. P. 261-375.

35. Toennies J.P. Scattering of molecular beams from surfaces // Appl. Phys. 1974. V. 3. № 2. P. 91114.

36. Weinberg W.H. Molecular beam scattering from solid surfaces // Adv. Colloid Interface Science. 1975. V. 4. № 4. P. 301-347.

37. Gerber R.B. Molecular scattering from surfaces: theoretical methods and results // Chem. Rev. 1987. V. 87. № 1. P. 29-79.

38. Kleyn A.W. Basic mechanisms in atom-surface interactions. In: Handbook of Surface Science. Vol. 3. Eds. E. Hasselbrink, B.I. Lundqvist. Amsterdam: Elsevier Science, 2008. Ch. 2, P. 29-52.

39. Goodman F.O. Quantum mechanical basis for the cubes models in gas-surface scattering theory, and an experimental test // J. Chem. Phys. 1970. V. 53. № 6. P. 2281-2283.

40. Ewing J.J., Milstein R., Berry R.S. Curve crossing in collisional dissociation of alkali halide molecules // J. Chem. Phys. 1971. V. 54. № 4. P. 1752-1760.

41. Brumer P. Combination rules and correlations in repulsive potential parameters for alkali halide diatomics // Phys. Rev. A. 1974. V. 10. № 1. P. 1-8.

42. Rittner E.S. Binding energy and dipole moment of alkali halide molecules // J. Chem. Phys. 1951. V. 19. № 8. P. 1030-1035.

43. Brumer P., Karplus M. Perturbation theory and ionic models for alkali halide systems. I. Diatomics // J. Chem. Phys. 1973. V. 58. № 9. P. 3903-3918.

44. Patil S.H. Interionic potentials in alkali halides // J. Chem. Phys. 1987. V. 86. № 1. P. 313-320.

45. Русин Л.Ю., Севрюк М.Б. Аннотированная библиография статей по потенциалам взаимодействия в молекулах галогенидов щелочных металлов и по потенциалам взаимодействия атомов инертных газов с катионами щелочных металлов, анионами галогенов и атомами ртути. Отчет в ЦИТиС. Регистрационный номер АААА-Б16-216092340017-7. М.: ИНЭПХФ РАН им. В.Л. Тальрозе, 2016. 276 с.

46. Кнунянц И.Л. (гл. ред.). Химическая энциклопедия. Т. 1. М.: Советская энциклопедия, 1988. $624 \mathrm{c}$.

47. Зефиров Н.С. (гл. ред.). Химическая энциклопедия. Т. 5. М.: Большая Российская энциклопедия, 1998. $784 \mathrm{c.}$

48. Русин Л.Ю., Севрюк М.Б., Азриель В.М., Акимов В.М., Кабанов Д.Б. Сравнительный анализ моделирования столкновительно-индуцированной диссоциации в системе $\mathrm{Xe}+\mathrm{CsBr}$ с двумя разными потенциалами взаимодействия Хе- $\mathrm{Br}^{-}$. Отчет в ЦИТиС. Регистрационный номер 216032240003. М.: ИНЭПХФ РАН им. В.Л. Тальрозе, 2016. 70 с.

49. Burroughs J.A., Wainhaus S.B., Hanley L. Impulsive excitation of $\mathrm{FeCp}_{2}^{+}$and $\mathrm{SiMe}_{3}^{+}$during surface-induced dissociation at organic multilayers // J. Chem. Phys. 1995. V. 103. № 15. P. 67066715.

50. Wilson K.R., Herschbach D.R. Molecular beam kinetics: Transition between rebound and stripping mechanisms in reactions of alkali atoms with polyhalide molecules // J. Chem. Phys. 1968. V. 49. № 6. Р. 2676-2683.

51. Ван дер Варден Б.Л. Математическая статистика. М.: ИЛ, 1960. 435 с. (Van der Waerden B.L. Mathematische Statistik. Berlin: Springer, 1957. ix+360 S.)

52. Крамер Г. Математические методы статистики. М.: Мир, 1975. 648 с. (Cramér H. Mathematical Methods of Statistics. Princeton: Princeton Univ. Press, 1946. xvi+575 p.)

53. Благовещенский Ю.Н. Тайны корреляционных связей в статистике. М.: Научная книга. ИНФРА-М («Библиотека Солев»), 2009. 158 с. 
54. Лагутин М.Б. Наглядная математическая статистика. М.: Бином. Лаборатория знаний, 2011. $472 \mathrm{c}$.

55. Русин Л.Ю., Севрюк М.Б., Тоеннес Я.П. Эффективные процедуры расчета корреляционных связей методом квазиклассических траекторий // Хим. физика. 2005. Т. 24. № 3. С. 3-11.

56. Русин Л.Ю., Севрюк М.Б., Колесникова Л.И. Корреляционный анализ столкновительной диссоциации пары двухатомных молекул с ионной связью. Отчет во ВНТИЦ. Инвентарный номер 02200900518. М.: ИНЭПХФ РАН, 2008. 134 с.

57. Азриель В.М., Акимов В.М., Русин Л.Ю., Севрюк М.Б. Оптимизация параметров поверхности потенциальной энергии системы $\mathrm{CsCl}+\mathrm{RbI}$ с помощью линейного регрессионного анализа // Хим. физика. 2010. Т. 29. № 5. С. 3-19.

58. Kuščer I. Reciprocity in scattering of gas molecules by surfaces // Surface Science. 1971. V. 25. № 2. P. 225-237.

59. Cercignani C. Scattering kernels for gas-surface interactions // Transport Theory Stat. Phys. 1972. V. 2. № 1. P. 27-53.

60. Cercignani C. The Boltzmann Equation and Its Applications. New York: Springer Science, 1988. xiii+455 p.

61. Liang T., Li Q., Ye W. A physical-based gas-surface interaction model for rarefied gas flow simulation // J. Comput. Phys. 2018. V. 352. P. 105-122.

62. Maxwell J.C. On stresses in rarified gases arising from inequalities of temperature // Philos. Trans. R. Soc. London. 1879. V. 170. P. 231-256.

63. Epstein M. A model of the wall boundary condition in kinetic theory // AIAA J. 1967. V. 5. № 10. P. 1797-1800.

64. Cercignani C., Lampis M. Kinetic models for gas-surface interactions // Transport Theory Stat. Phys. 1971. V. 1. № 2. P. 101-114.

65. Сажин О.В., Кулёв А.Н. Программный комплекс для тестирования способа моделирования рассеяния газовых молекул поверхностью // Матем. моделирование. 2006. Т. 18. № 12. С. 107114. Поправка: 2007. Т. 19. № 5. С. 127.

66. Sazhin O.V., Kulev A.N., Borisov S.F., Gimelshein S. Numerical analysis of gas-surface scattering effect on thermal transpiration in the free molecular regime // Vacuum. 2007. V. 82. № 1. P. 20-29.

67. Ковалёв В.Л., Якунчиков А.Н. Анализ моделей рассеяния на основе результатов траекторных расчетов // Изв. РАН. МЖГ. 2012. № 5. С. 80-87.

68. Yakunchikov A.N., Kovalev V.L., Utyuzhnikov S.V. Analysis of gas-surface scattering models based on computational molecular dynamics // Chem. Phys. Lett. 2012. V. 554. P. 225-230.

69. Ковалёв В.Л., Якунчиков А.Н. Исследование рассеяния газа на поверхности с помощью молекулярно-динамического моделирования и сравнение результатов с теоретическими моделями //Физико-химическая кинетика в газовой динамике. 2013. Т. 14, вып. 3. http://chemphys.edu.ru/issues/2013-14-3/articles/415/ (6 c.).

70. Liang T., Li Q., Ye W. Performance evaluation of Maxwell and Cercignani-Lampis gas-wall interaction models in the modeling of thermally driven rarefied gas transport // Phys. Rev. E. 2013. V. 88. № 1. Paper 013009 (11 p.).

71. Bärwinkel K., Rottmann L. A model for irreversible scattering of gas atoms on a metal surface // Surface Science. 1993. V. 287/288. Part 1. P. 35-38. 